\title{
Modeling and Simulation of Gas Centrifuge Cascades for Enhancing the Efficiency of IAEA Safeguards
}

\author{
A Dissertation \\ Presented to \\ the faculty of the School of Engineering and Applied Science \\ University of Virginia \\ in partial fulfillment \\ of the requirements for the degree
}

Doctor of Philosophy

by

Patrick James Migliorini

May 2013 
Approval Sheet

The dissertation

is submitted in partial fulfillment of the requirements

for the degree of

Doctor of Philosophy

Patrick James Migliorini

The dissertation has been read and approved by the examining committee:

\begin{tabular}{c}
\hline Houston G. Wood, Advisor \\
\hline Robert J. Ribando, Committee Chair \\
\hline Harsha K. Chelliah \\
Michael E. Gorman
\end{tabular}

Todd S. Sechser

Accepted for the School of Engineering and Applied Science:

Dean, School of Engineering and Applied Science

May 2013 


\section{Abstract}

Since its inception in 1957, the International Atomic Energy Agency (IAEA) has been tasked with ensuring the peaceful uses of nuclear facilities in nation-states that have ratified the Treaty on the Non-Proliferation of Nuclear Weapons (NPT). To achieve this goal, the IAEA employs safeguards techniques to verify the declared use of facilities related to nuclear power. Recently, there has been an increasing interest and demand for nuclear power throughout the world while the budget of the IAEA has remained fairly stagnant. In order for the IAEA to continue to meet their verification goals, there has been an emphasis to enhance the effectiveness and efficiency of safeguards, most notably at gas centrifuge enrichment plants. Most efforts have focused on reducing the level of on-site inspection activities through the development of new technologies for unattended process monitoring. In this research, computational models are developed to study the dynamics and capabilities of gas centrifuge cascades and address the verification goals of the IAEA in the following areas:

One safeguards tool that IAEA inspectors employ during on-site inspections is environmental sampling - analyzing swipe samples from a plant to determine enrichment levels. Discrepancies between measured and expected values of enrichment may not always indicate misuse of a cascade. Here, a method to quantify reasonable levels of enrichment in a cascade due to normal operating transients is developed. This knowledge can help reduce the number of false alarms created by anomalies and the number of on-site inspection activities.

When analyzing the capabilities of a cascade, it is necessary to know the separative performance of a gas centrifuge over a range of operating conditions. Typically, the knowledge of centrifuge geometry and operational parameters are required to characterize a machine. Due to the sensitive nature of gas centrifuge research and development, it is 
often the case that this information is not known. A novel, semi-empirical method for calculating the separative power and separation factor of a gas centrifuge is developed. The method is verified through a comparative study with results from the Pancake code.

In the event that a nation decides to withdraw from the NPT, it is important to understand the capabilities of an enrichment program to develop enough enriched material for a nuclear weapon. A method to study the cascade interconnection scenario is developed and is used to analyze the Fuel Enrichment Plant in Iran. The importance of including inefficiencies in a capability study is shown.

Unattended monitoring systems are being developed to allow the IAEA to draw safeguards conclusions with less effort. Computational modeling can offer insight into the dynamics of a cascade allowing these technologies to be used in a more efficient way. A transient fluid dynamics and isotope separation model is developed to study signatures of misuse and time-frames associated with the transition between normal and off-normal operating states. Illustrative results show potential indicators of intentional misuse and the time that detectable phenomena remain in the cascade.

The approaches and ideas developed in this dissertation are generic and can be applied to any cascade and enrichment plant. 


\section{Acknowledgments}

My pursuit of a doctoral degree in engineering, while taxing at times, has been an extremely rewarding and enjoyable experience. This was not a journey made by my own effort alone and for that there are many people who I owe a great deal of gratitude.

First and foremost, I would like to thank my advisor, Dr. Houston Wood, for accepting me as a graduate student and for introducing me to the interesting field of nuclear nonproliferation and safeguards, for that I am in his debt. Houston has been a great mentor, supporter, story-teller, and friend. I am fortunate to have an advisor who provided so much opportunity to attend conferences, meetings, and training courses taking me to places all around the US and Europe.

I would also like to thank my second boss, Dr. Robert Ribando. For more semesters than I can remember, I was a teaching assistant for Bob's Heat Transfer class. Working for Bob was a pleasure and I thank him for giving me the freedom to teach and grade in my own style. I would also like to thank Bob for serving as my dissertation committee chair.

I would also like to extend a sincere thank you to Dr. Harsha Chelliah, Dr. Michael Gorman, and Dr. Todd Sechser for serving on my dissertation committee and showing an interest in my research.

I thank Michael Whitaker at Oak Ridge National Laboratory for serving as my mentor during my internships at the lab. He has been a source of much insight into the world of international safeguards and has provided the motivation for much of this work. I thank David Albright at the Institute for Science and International Security for interesting discussions and for motivating my work in nonproliferation.

I owe many thanks to the other graduate students and research professionals I have worked with throughout my studies, especially Alex Untariou, Chuck Witt, and Neal Morgan. Alex has been a great colleague and our discussions always seem to lead to 
interesting research projects. In the short time we've worked together, Chuck has amazed me with his work ethic and talent. I owe him for finding errors in my work; I hope together we have caught them all. Neal has made our window-less office in MEC a little more tolerable and a lot more colorful.

Finally, I would like to thank my friends and family, who have supported me through this experience - my house-mates John Duda, Russell Biagi, Josh Codoni, and Brendyn Sarnacki, my mom Ingrid, dad Peter, and sister Rachel. My mom and dad have always been a great source of inspiration and support; they have always encouraged me to work hard and pursue my passions.

Lastly (but not leastly...), I owe a huge thanks to my fiancée (and soon-to-be wife) Katie, for her patience, love, and encouragement. She has kept me a sane and well-balanced engineer.

I am grateful to the Nuclear Engineering Science Laboratory Synthesis internship program for summer support and resources and Oak Ridge National Laboratory for partially supporting this research. 


\section{Contents}

List of Figures $\quad$ vi

List of Tables $\quad$ xi

Acronyms $\quad$ xii

1 Introduction $\quad 1$

1.1 Historical Context . . . . . . . . . . . . . . . . . . . 1

1.2 Nuclear Nonproliferation and the IAEA . . . . . . . . . . . . . 1

1.3 The Nuclear Fuel Cycle and Uranium Enrichment . . . . . . . . . . . . . 3

1.4 IAEA Safeguards at Gas Centrifuge Plants . . . . . . . . . . . . . . . . 5

1.4.1 Current Safeguards Technologies . . . . . . . . . . . 7

1.4.2 Next Generation Safeguards Technologies and Approaches . . . . . 8

1.4.3 Breakout Scenarios . . . . . . . . . . . . . . . 9

1.5 Outline of Dissertation and Objectives . . . . . . . . . . . . . . 9

\section{The Gas Centrifuge:}

Internal Flow and Isotope Transport $\quad 13$

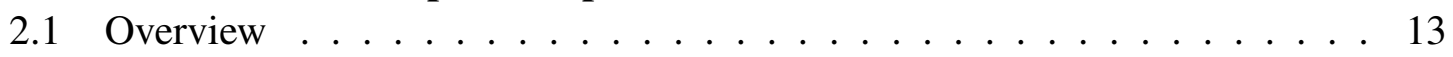

2.1.1 Review of Flow Solution Methods . . . . . . . . . . . . . . 15

2.2 Governing Equations . . . . . . . . . . . . . . . . . . . . 19

2.2.1 Linearization Process . . . . . . . . . . . . . . . 21

2.2.2 Non-Dimensionalization . . . . . . . . . . . . . 23

2.3 Onsager's Model . . . . . . . . . . . . . . . . . . . 24

2.4 The Pure-Axial Flow Model . . . . . . . . . . . . . . . . . . 28

2.5 Isotope Transport . . . . . . . . . . . . . . . . . . . 31

2.5.1 Deriving the Gradient Equation Through Radial Averaging . . . . . 32

2.5.2 Separation Parameters . . . . . . . . . . . . 36

3 Cascade Theory $\quad 42$

3.1 Overview .............................. 42

3.2 General Cascades . . . . . . . . . . . . . . . . . . 43

3.2.1 Approximations to the Gradient Equations . . . . . . . . . . . . . . 47

3.2.2 Limiting Conditions . . . . . . . . . . . . . . . . . . . 48

3.3 The Ideal Cascade . . . . . . . . . . . . . . . . . . . . . . . . . . 48

4 GCEPs and Proliferation $\quad \mathbf{5 1}$

4.1 Components of a GCEP . . . . . . . . . . . . . . . . . 51

4.2 Proliferation and Misuse of a GCEP . . . . . . . . . . . . . . . 53

4.3 Relevant Work . . . . . . . . . . . . . . . . 56 
5 Quantifying Reasonable Enrichment Levels in a Cascade 59

5.1 Overview . . . . . . . . . . . . . . . . . . . 59

5.2 Cascade Design and Off-Normal Operation . . . . . . . . . . 60

5.3 Results . . . . . . . . . . . . . . . . . . . . . . . . . . 64

5.3.1 Centrifuge Performance . . . . . . . . . . . . . . 64

5.3.2 Designed Cascade Normal Operation _ . . . . . . . . . . . 65

5.4 Summary . . . . . . . . . . . . . . . . . . . 68

6 Semi-Empirical Method of Calculating a Centrifuge Performance Map 71

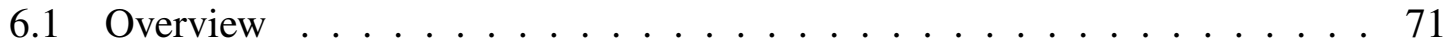

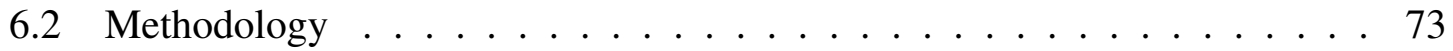

6.3 Results . . . . . . . . . . . . . . . . . . 76

6.3 .1 Case Studies . . . . . . . . . . . . . . . . . . 76

6.3.2 Centrifuge Level Comparison _. . . . . . . . . . . . . . . 77

6.3 .3 Cascade Level Comparison . . . . . . . . . . . . . . . . . . 78

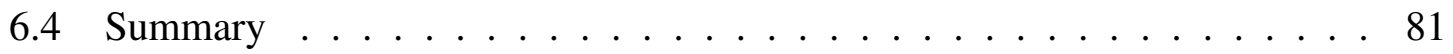

7 A Fixed Plant Method for Quantifying Breakout Times 83

7.1 Overview . . . . . . . . . . . . . . . . . 83

7.2 Multi-Step Enrichment Processes . . . . . . . . . . . . . . . . . . . . 84

7.3 Iran's Natanz Fuel Enrichment Plant . . . . . . . . . . . . . . . 86

7.4 IR-1 Centrifuge Performance . . . . . . . . . . . . . . . . . 88

7.5 Enrichment Plant Capabilities and Breakout Time . . . . . . . . . 88

7.6 Summary . . . . . . . . . . . . . . . . . . . . 91

8 Transient Modeling of Gas Centrifuge Cascades 94

8.1 Overview . . . . . . . . . . . . . . . . . . . . . 94

8.2 Transient Flow Modeling . . . . . . . . . . . . . . . . . . . . 95

8.2 .1 Simscape . . . . . . . . . . . . . . . . . . . . 95

8.2.2 Modeling a Gas Centrifuge Cascade . . . . . . . . . . . . . 98

8.3 Transient Isotope Separation Model . . . . . . . . . . . . . . . . . . 102

8.4 Model Implementation . . . . . . . . . . . . . . . . . . . . . 106

8.4.1 Off-Normal Operation Specification . . . . . . . . . . . . . 108

8.5 Model Verification . . . . . . . . . . . . . . . . . . . . . . . . . 112

8.5.1 Lumped Pipe Model . . . . . . . . . . . . . . . . . . . . . 112

8.5 .2 Flow Model . . . . . . . . . . . . . . . . . . . . . . . . . . 114

8.6 Case Summaries . . . . . . . . . . . . . . . . . . . . . . . . . . . . . 115

8.7 Simulation Results . . . . . . . . . . . . . . . . . . . . . 118

8.7.1 Signatures of Misuse in a Cascade . . . . . . . . . . . . 118

8.7.2 Off-Normal Operation Time Frames . . . . . . . . . . . . . 122

8.8 Summary . . . . . . . . . . . . . . . . . . . . . . . . . 124

9 Summary and Future Work $\quad 126$

9.1 Summary of Dissertation and Contributions . . . . . . . . . . 126

9.2 Future Work and Direction . . . . . . . . . . . . . . . . 128 
$\begin{array}{ll}\text { Bibliography } & 130\end{array}$

Appendix A: Coefficients of Pure Axial Flow Model 139

Appendix B: Full Cascade Details for Transient Study 140 


\section{List of Figures}

1.1 Major nuclear flows at a generic gas centrifuge enrichment plant. The flow of material begins by bringing in feed cylinders full of solid uranium hexafluoride $\left(\mathrm{UF}_{6}\right)$. These cylinders are heated to convert the solid to a gas. Next, the gaseous $\mathrm{UF}_{6}$ is fed into the cascades where the material is enriched and collected into product and tails cylinders. The product cylinders are transported off-site and the tails cylinders remain in storage.

1.2 The time evolution of: (a) Significant Quantities (SQs) of nuclear material under IAEA safeguards, (b) the budget of the IAEA, (c) the safeguards budget per SQ. Due to the large increase of nuclear material under safeguards coupled with the stagnant budget of the IAEA, the safeguards budget per SQ has dropped from nearly $\$ 15,000$ per SQ to under $\$ 300$ per SQ. This budgetary constraint has led to an effort to perform more efficient safeguards activities. . . . . . . . . . . . . . .

2.1 A schematic diagram of a countercurrent gas centrifuge. The centrifuge is essentially a hollow cylinder spun rapidly about its axis. This motion induces a rigid body rotation of the gas contained in the cylinder, creating a large pressure gradient between the axis and the wall. The pressure gradient drives a concentration gradient where the lighter isotope tends towards the axis and the heavier isotope towards the wall. This radial separation effect can be enhanced by inducing an axial current. . . . . . . . . . . . . . . . 14

2.2 Schematic drawings of the (a) Zippe centrifuge and (b) Groth ZG5 centrifuge. The axial countercurrent in the Zippe centrifuge is driven by a thermal gradient between the top and bottom end caps. The flow in the Groth centrifuge is driven by the drag of the waste scoop. . . . . . . . . 16

2.3 A typical flow diagram in a thermally driven gas centrifuge. Boundary layers form on the top and bottom endcaps (Ekman layers) and the side wall (Stewartson layer). In the top Ekman layer, colder, denser fluid particle are driven to the outside wall and down the centrifuge wall. In the bottom Ekman layer, warmer, lighter fluid particles are driven towards the axis and rise up the centrifuge in the inner, inviscid core. This flow is evident in the axial velocity and mass flux profiles. . . . . . . . . . . . . . . 18 
2.4 1D performance maps for the Rome and Iguaçu centrifuge. 1D performance maps detail the dependence of the separative power (red $\mathrm{x}$ ) and the separation factor (blue circle) versus the feed rate. For this case, the centrifuge cut is $\theta=0.5$. Typically, the separative power of the gas centrifuge approaches zero as the feed rate goes to zero, increases with increasing feed rate to a maximum point and then tails off. The feed rate where the separative power is maximized is deemed the optimal point. The separation factor reaches a maximum as the feed rate goes to zero and decreases with increasing feed rate. . . . . . . . . . . . . . . . 40

2.5 2D performance maps for the Rome centrifuge. 2D performance maps detail the separative performance of the centrifuge over a range of feed rates and cuts. The combination of feed rate and cut that maximize the centrifuge separative power is deemed the optimal operating point. . . . . . 40

$2.62 \mathrm{D}$ performance maps for the Iguaçu centrifuge . . . . . . . . . . . . . 41

3.1 Flow diagrams for a countercurrent cascade. Centrifuges are connected in parallel into stages where the individual centrifuge flows are collected into header piping. Stages are connected in series with the product stream of a stage feeding the next stage and the tails stream of a stage feeding the previous stage. The enriching section of the cascade includes the feed stage and all stages above. The stripping section includes all stages below the feed stage. . . . . . . . . . . . . . . . . . . . 4

4.1 Flow diagram of a generic gas centrifuge enrichment plant. Feed cylinders are transported from the cylinder storage area to an autoclave where solid $\mathrm{UF}_{6}$ is heated and converted to a gas. The gaseous $\mathrm{UF}_{6}$ is pumped though a sampling port and impurity removal station and into a holdup drum. The pressure of the gas is reduced and then fed into the cascades. Cascades are typically joined together in production units. Depending on the size of the plant, there can be one or several production units. The product flows of the cascades are transported through a product header and collected in product cylinders. Similarly, the tails flows are collected and stored on-site. . . . . . 52

4.2 Flow diagram of a real, one-up, one-down symmetric cascade. Typically, centrifuges are arranged in rows with the stages created by piping. In this example, an 8 stage cascade is formed with 40 centrifuges. . . . . . . . . 53

4.3 Flow diagram of a generic stage in a cascade. Centrifuges are linked to header piping by small pipes. The feed material enters the stage and is distributed to each centrifuge. The product and tails header pipes collect the individual product and tails streams from each centrifuge. . . . . . . . 54 
4.4 Generic proliferation scenarios that may occur in a GCEP. a) One way to increase the product enrichment out of the cascade is to lengthen the cascade by reconfiguring the cascade. Centrifuges can be taken from the middle stages of the cascade and used to add stages to the top of the cascade. b) Another way to increase the cascade product enrichment is to partially or fully recycle the product stream into either the feed stage or some other stage of the cascade. c) The most efficient way to produce a higher enrichments in a cascade hall is to connect cascades in parallel and series. d) Overproduction or diversion of material can be achieved by introducing side-streams through sampling ports in a stage. . . . . . . . . 55

5.1 Performance maps for the Iguaçu centrifuge. Case 1 represents a normally operating centrifuge that has been optimized at the designed feed rate. Case 2 represents a centrifuge that is intentionally operated off-normally. At each feed rate, the scoop drag and temperature gradient are varied to optimize the centrifuge separative power at each feed rate.

5.2 Cascade product concentrations versus the scaled product rate for: a) ${ }^{232} \mathrm{U}$, b) ${ }^{234} \mathrm{U}$, c) ${ }^{235} \mathrm{U}$, d) ${ }^{236} \mathrm{U}$. Cases $\mathrm{ON}$ and OR represent the range of enrichments that can be achieved in a normally operating cascade. The maximum capability of a cascade that has not been reconfigured are represented in Cases RN and RR. . . . . . . . . . . . . . . . .

$5.3{ }^{234} \mathrm{U}$ concentration versus ${ }^{235} \mathrm{U}$ concentration over the entire range of concentrations achievable. Because the ${ }^{234} U$ is lighter than ${ }^{235} U$, the ${ }^{234} U$

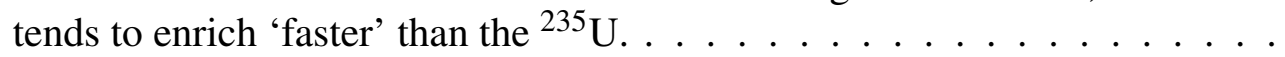

$5.4{ }^{236} \mathrm{U}$ concentration versus ${ }^{235} \mathrm{U}$ concentration over the entire range of concentrations achievable. Because the ${ }^{236} \mathrm{U}$ is lighter than ${ }^{235} \mathrm{U}$, the ${ }^{236} \mathrm{U}$ tends to enrich 'slower' than the ${ }^{235} \mathrm{U} . \ldots \ldots \ldots$

6.1 Cascade flow diagram for a theoretical cascade model of a gas centrifuge. The theoretical cascade is characterized by a number of stages, stage upflow rate, and stage enrichment factor. . . . . . . . . . . . . . 73

6.2 Algorithm flow chart for calculating centrifuge performance by treating the centrifuge as a square cascade. Target separation parameters are sought through an iterative procedure by solving the exact gradient equations over a range of feed rates and cuts. . . . . . . . . . . . . .

6.3 Comparison of the predicted centrifuge separative power versus feed rate for: a) $\theta=0.05$, b) $\theta=0.275$, c) $\theta=0.50$, d) $\theta=0.725$, e) $\theta=0.949$. Good agreement is found between the semi-empirical model and Pancake for typically operating conditions $(\theta=0.50)$. Over the entire range, the average percent difference is $14.25-18.30 \%$. . . . . . . . . . . . . 79

6.4 Comparison of the predicted centrifuge separation factor versus feed rate for: a) $\theta=0.05$, b) $\theta=0.275$, c) $\theta=0.50$, d) $\theta=0.725$, e) $\theta=0.949$. Very good agreement is found between the two models for the entire range of operating conditions. The average percent difference is $1.15-1.49 \%$. . . . 
7.1 Black box treatment of a cascade hall in an ideal separative work analysis. The separative power of all the centrifuges is summed and the separative power equation is used to predict the total product rate. This method neglects mixing inefficiencies that are inherent to real cascade operation. . . 84

7.2 Schematic of a multi-step enrichment process via cascade interconnection. Cascades are joined into parallel groupings called steps. Each step is then joined in series much like centrifuges and stages. . . . . . . . . . . .

7.3 Methodology for calculating the performance of a multi-step enrichment process. For a given step and cascade configuration, the exact gradient equations are solved based on target product enrichments for each step. The product rate of each step is used as the feed of the next step. The product rate of the top step is the production rate for the multi-step enrichment process and is used to calculate breakout time. . . . . . . . . . . . . . 87

7.4 2D centrifuge performance maps for the IR-1 centrifuge. The performance maps are developed using the semi-empirical method developed in Chapter 6 with target parameters derived from the IAEA safeguards report. . . . . .

8.1 Diagram of the lumped parameter pipe model developed in Simscape. The pipe model accounts for fluid compressibility and viscous pressure loss. . .

8.2 Diagram of a cascade stage model with specified stage upflow rate. The centrifuge is modeled as a volume connected to two ideal compressors. The upflow and downflow through the centrifuge is determined by a userspecified upflow rate. Downstream of the centrifuge is a diversion pathway and a header pipe that leads to the next stage. . . . . . . . . . . . . 100

8.3 Flow diagram of a general cascade stage. Feed material enters the stage through a feed point and into an associated stage holdup. The enriched upflow stream travels through a withdrawal point, into a header pipe, and to the next stage. Similarly, the downflow stream is transported to the previous

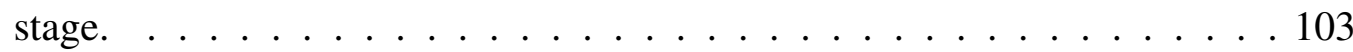

8.4 Flow chart for a typical TransCasc simulation. The program associated with each step of the simulation is labeled in blue. . . . . . . . . . . . . 108

8.5 Off-normal operation flow specifications in TransCasc, a) Upflow and cut scale factor, b) Upflow withdrawal rate factor, c) Cascade feed rate factor, d) Centrifuge scale factor. The upflow and cut scale factor are used to control the stage flow rates. They cannot be used together as this would over-define the simulation. The upflow withdrawal rate factor controls the amount of material withdrawn from the stage upflow. The cascade feed rate factor controls the cascade feed rate. The centrifuge scale factor controls the number of centrifuges in each stage. . . . . . . . . . . . . . 112

8.6 Results of the lumped pipe model verification study. A simulation is run where the stage upflow rates are reduced by half over a fifteen minute period. The stage feed rates a) and average stage concentrations b) are compared for cases where 2, 3, and 5 segments are used in the pipe model. The verification study shows that 3 segments are sufficient. . . . . . . . . . 113 
8.7 Results of the flow model verification study. The cascade feed rate is increased by $10 \%$ over a one minute period. The cascade tails rate a) and the Stage 5 upflow rates b) are compared with data from a model developed by Rousseau et. al. The results of the verification show good agreement in the transient response. The difference between normal and off-normal flow rates is a result of retrieving the data from a plot using Data Thief III. . . . 116

8.8 Cascade product and tails rates and concentrations for the Iguaçu2 cascade, Cases 1-8. The values are normalized by the normal cascade rates and concentrations. Cases 1-4, centrifuge failure in the feed stage, represent unintentional off-normal operation of the cascade. Cases 5-8 represent intentional misuse of the cascade by introducing undeclared feed. A clear difference is shown in the cascade dynamics between the two scenarios. . . 119

8.9 Cascade product and tails rates and concentrations for the Iguaçu2 cascade, Cases 9-12. The values are normalized by the normal cascade rates and concentrations. These cases represent scenarios that should be detected by unattended monitoring systems as the change in the concentrations is large. 120

8.10 Instantaneous flow balances for the Iguaçu2 cascade, a) Total flow balance, b) ${ }^{235} \mathrm{U}$ flow balance. The imbalance of the flow rates is relatively short while the imbalance of ${ }^{235} \mathrm{U}$ requires significantly more time to dissipate. As expected, Cases 5-6 show a constant imbalance due to the undeclared feed. . . . . . . . . . . . . . . . . . . . . 121

8.11 Normalized ratio of the minor isotopes in the cascade product stream to ${ }^{235} \mathrm{U}$, a) ${ }^{232} \mathrm{U}$, b) ${ }^{234} \mathrm{U}$, c) ${ }^{236} \mathrm{U}$. The difference between the intentional misuse cases and centrifuge failure is clearly seen in the behavior of the

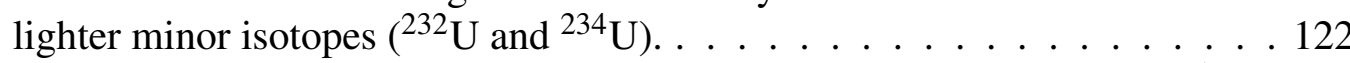

8.12 Normalized ratio of the minor isotopes in the cascade tails stream to ${ }^{235} \mathrm{U}$, a) ${ }^{232} U$, b) ${ }^{234} U$, c) ${ }^{236} U$. The minor isotope ratios in the tails stream do not differ significantly between the off-normal scenarios. . . . . . . . . . . 123

8.13 Time-frames of transitions between normal and off-normal operational modes. For most cases, the transition time is greater than the notification time for Short Notice Random Inspections. Detectable phenomena will remain in the cascade long enough for discovery using other measurement

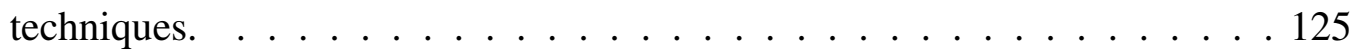




\section{List of Tables}

1.1 Typical uranium isotope concentrations in commercial natural uranium . . . 3

1.2 IAEA definitions of significant quantities and timeliness goals . . . . . . 6

2.1 Radial boundary conditions for the Onsager Pancake equation. . . . . . . . 28

2.2 Separation terms in the gradient equation (Equation (2.78)) . . . . . . . 35

2.3 Geometric and operating details of the Rome and Iguaçu centrifuges . . . . 38

5.1 Typical uranium isotope concentrations in commercial natural uranium . . . 61

5.2 Iguaçu centrifuge optimal operating conditions . . . . . . . . . . . . 64

5.3 Designed cascade, normal operating parameters . . . . . . . . . . 66

5.4 Designed cascade, normal operating product concentrations . . . . . . . . 66

6.1 Case summaries and calculated optimal design points . . . . . . . . . . . . 77

6.2 Percent differences between each case and Pancake averaged overall all feed rates for the predicted separative power (in \%) . . . . . . . . . . . 79

6.3 Percent differences between each case and Pancake averaged overall all feed rates for the predicted separation factor (in \%) . . . . . . . . . . 80

6.4 Design cascade based on Pancake performance map . . . . . . . . . . . . . 81

6.5 Percent difference between all cases and Pancake for the predicted cascade product concentration, cascade tails concentration, and separative power . . 82

7.1 Estimated operating characteristics for the IR-1 centrifuge . . . . . . . . 89

7.2 Cascade structure for each enrichment step for the 15 and 17 stage cascades 90

7.3 Summary of cascade results for Case 1: 15 Stage Cascade, No Inventory . . 92

7.4 Summary of cascade results for Case 2: 15 Stage Cascade, With Inventory . 92

7.5 Summary of cascade results for Case 3: 17 Stage Cascade, No Inventory . . 93

7.6 Summary of cascade results for Case 4: 17 Stage Cascade, With Inventory . 93

8.1 Cascade and centrifuge parameters used in comparison study to the Rousseau model . . . . . . . . . . . . . . . . . . . . . . . . . . 114

8.2 Stages, number of centrifuges, and upflow rates specified in verification study 115

8.3 Nominal cascade designs for transient study . . . . . . . . . . . . . . . 117

8.4 Simulation cases and off-normal specifications . . . . . . . . . . . . 118

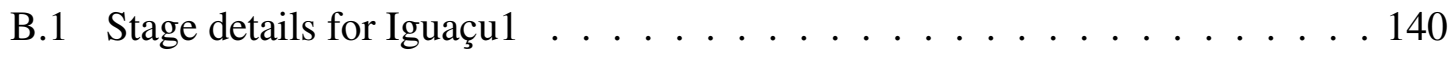

B.2 Stage details for Iguaçu2 . . . . . . . . . . . . . . . . . . 141

B.3 Stage details for Iguaçu3 . . . . . . . . . . . . . . . . . . . . 141

B.4 Stage details for Rome1 . . . . . . . . . . . . . . . . . . . . . . . . . . . . . . . . . . . . . . . . . . . . . . .

B.5 Stage details for Rome2 . . . . . . . . . . . . . . . . . . . . 142 


\section{Acronyms}

\begin{tabular}{|c|c|}
\hline IAEA & International Atomic Energy Agency \\
\hline NNWS & Non-Nuclear Weapon States \\
\hline NWS & Nuclear Weapon States \\
\hline $\mathrm{NUF}_{6}$ & Natural Uranium Hexafluoride \\
\hline INFCIRC & Information Circular \\
\hline NPT & Treaty on the Non-Proliferation of Nuclear Weapons \\
\hline $\mathrm{UF}_{6}$ & Uranium Hexafluoride \\
\hline SQ & Significant Quantity \\
\hline LEU & Low Enriched Uranium \\
\hline HEU & High Enriched Uranium \\
\hline GCEP & Gas Centrifuge Enrichment Plant \\
\hline DU & Depleted Uranium \\
\hline NU & Natural Uranium \\
\hline HSP & Hexapartite Safeguards Project \\
\hline LFUA & Limited Frequency Unannounced Inspections \\
\hline PIV & Physical Inventory Verification \\
\hline NDA & Non-Destructive Assay \\
\hline DA & Destructive Assay \\
\hline ES & Environmental Sampling \\
\hline CEMO & Continuous Enrichment Monitor \\
\hline WGU & Weapon Grade Uranium \\
\hline
\end{tabular}




\section{Chapter 1}

\section{Introduction}

\subsection{Historical Context}

Arguably one of the most dichotomous developments of humankind, harnessing nuclear energy has been the focus of many political debates, scientific research, and dinnertime conversation since the discovery of nuclear fission in 1939. Nuclear fission has been a major contributer to the advancement of humankind through applications in the energy market, medical field, and agriculture. As much benefit as nuclear power has provided, it has also shown to be a shear destructive force that can level whole cities in the blink of an eye. Since the first use of a nuclear weapon in 1945, the fear of a world with wide-spread nuclear war has been the motivation of many efforts to promote only the peaceful uses of nuclear energy and to prevent the spread of nuclear weapons.

In 1953, President Dwight D. Eisenhower delivered a speech to the United Nations entitled "Atoms for Peace," in which he proposed the creation of an organization to "serve the peaceful pursuits of mankind [1]." In the wake of this speech, with unanimous support from the General Assembly, the International Atomic Energy Agency (IAEA) was created in 1957 [2]. The main objectives of the IAEA are to promote the study, development, and application of peaceful nuclear energy by establishing and applying safeguards to verify that nuclear technology is not associated with military purposes.

\subsection{Nuclear Nonproliferation and the IAEA}

The Treaty on the Non-Proliferation of Nuclear Weapons (Non-Proliferation Treaty, NPT) is the most major agreement in the nuclear nonproliferation regime [3]. Entering into 
force in 1970, the NPT was designed to prevent the spread of nuclear weapons, encourage the development of technologies for peaceful uses of nuclear power, and to promote disarmament [3]. The NPT establishes two types of nation States, nuclear weapon States (NWS) and non-nuclear weapon States (NNWS). To be classified as a NWS, a State must have manufactured and detonated a nuclear weapon prior to January 1, 1967 [3]. Of the 190 parties to the treaty, only five States hold this status: China, France, the Russian Federation, the United Kingdom, and the United States [3]. There are currently four countries that are non-signatories: India, Israel, North Korea ${ }^{1}$, and Pakistan [4].

To enforce the goals of the NPT, the treaty establishes a safeguards system under the responsibility of the IAEA. Under the treaty, each NNWS must enter into a safeguards agreement with the IAEA to verify that the State is developing nuclear technologies only for peaceful uses and is not developing or pursuing nuclear weapons. The technical objectives of IAEA safeguards are:

"the timely detection of the diversion of significant quantities of nuclear material from peaceful nuclear activities to the manufacture of nuclear weapons or of other nuclear explosive devices or for purposes unknown, and the deterrence of such diversion by the risk of early detection [3]."

The generic term 'safeguards' refers to all measures applied by the IAEA to prevent the non-peaceful uses of nuclear material and the timely detection of diversion of nuclear material. The types of safeguards agreements between the IAEA and member States can vary in levels of comprehensiveness and verification methods authorized to the IAEA. Under the NPT and INFCIRC/66-type agreements, the IAEA employs verification methods that are largely based on nuclear material accountancy, the practice of verifying the inventory of declared nuclear material [5]. Under this agreement, the IAEA can also apply containment and surveillance measures. These safeguards techniques are applied by carrying out on-site inspections at all declared nuclear facilities in a State. These types

\footnotetext{
${ }^{1}$ North Korea was a member NNWS until January 10, 2003, when it withdrew from the treaty.
} 
Table 1.1: Typical uranium isotope concentrations in commercial natural uranium

\begin{tabular}{cc}
\hline Isotope & Mole Fraction $(\%)$ \\
\hline${ }^{232} \mathrm{U}$ & trace \\
${ }^{234} \mathrm{U}$ & 0.0062 \\
${ }^{235} \mathrm{U}$ & 0.72 \\
${ }^{236} \mathrm{U}$ & trace \\
${ }^{238} \mathrm{U}$ & 99.27 \\
\hline
\end{tabular}

of safeguards are deemed the 'traditional' approach and focus only on verifying nuclear material at declared facilities with significant nuclear activities. The disadvantage of this approach was brought to light with the discovery of Iraq's clandestine nuclear weapons program in 1991 [6].

In 1997, the IAEA Board of Governors approved the Additional Protocol, a framework which strengthens the IAEA's safeguards system through additional verification measures [7]. Under the Additional Protocol, the IAEA is granted broader access to facilities allowing them to verify the absence of undeclared nuclear material and activities in addition to non-diversion of material [7].

\subsection{The Nuclear Fuel Cycle and Uranium Enrichment}

Naturally occurring uranium ore contains two main isotopes ${ }^{235} \mathrm{U}$ and ${ }^{238} \mathrm{U}$ with trace amounts of three other isotopes (see Table 1.1). The most important isotope from a nuclear power perspective is the fissile isotope ${ }^{235} \mathrm{U}$.

For most nuclear reactors, the concentration of ${ }^{235} \mathrm{U}$ in natural uranium is not great enough to create a sustained nuclear reaction, and the concentration must be increased in a process called enrichment. Uranium enrichment can be performed by a variety of methods including: Gaseous Diffusion, Gas Centrifuge, Aerodynamic Methods (Vortex Tube and Separation Nozzle), Chemical and Ion Exchange, Laser Separation (Molecular and Atomic Vapor), and Electromagnetic Separation. Currently, the most popular method 


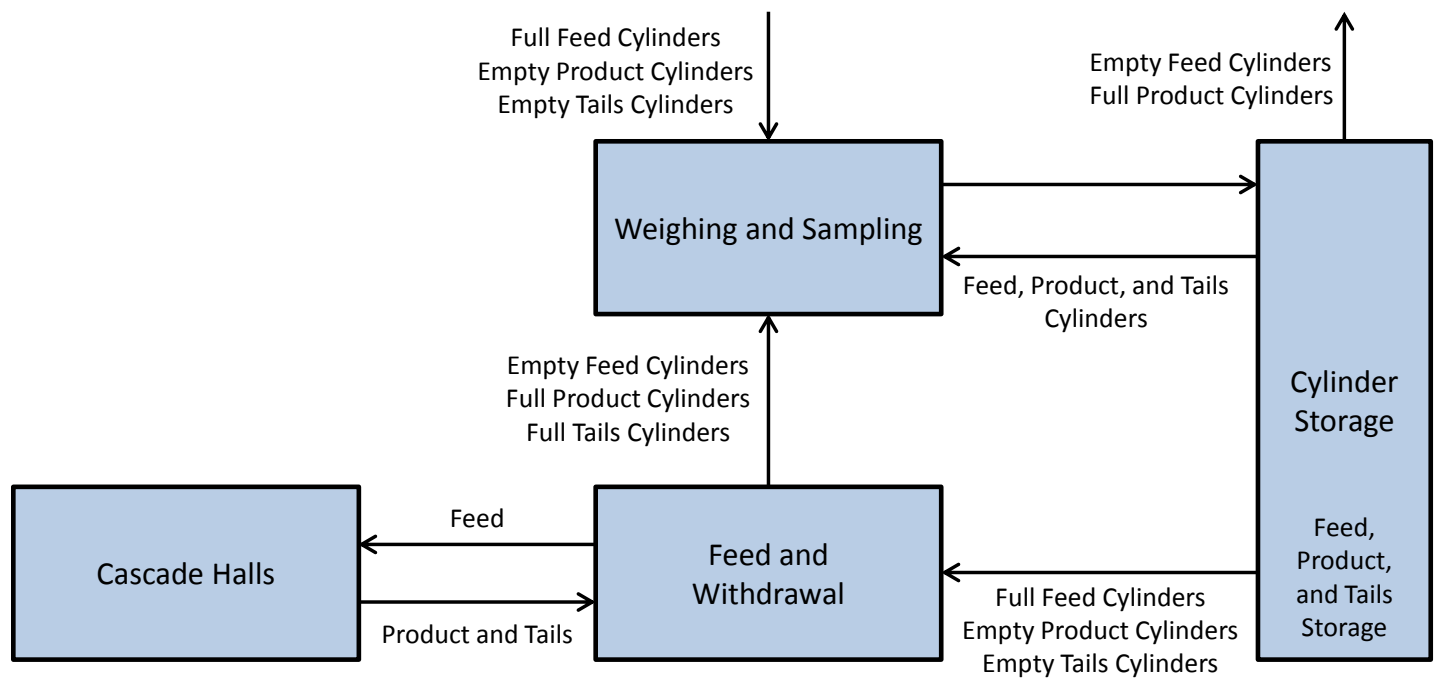

Figure 1.1: Major nuclear flows at a generic gas centrifuge enrichment plant. The flow of material begins by bringing in feed cylinders full of solid uranium hexafluoride $\left(\mathrm{UF}_{6}\right)$. These cylinders are heated to convert the solid to a gas. Next, the gaseous $\mathrm{UF}_{6}$ is fed into the cascades where the material is enriched and collected into product and tails cylinders. The product cylinders are transported off-site and the tails cylinders remain in storage.

for enrichment is the gas centrifuge due to a relatively high separation effect, low inventory, and low energy consumption [8]. For a nuclear power reactor, uranium must be enriched to roughly $3-5 \%{ }^{235} \mathrm{U}$, however, this same process can be used to enrich uranium to a level that can be used in a nuclear weapon $\left(>90 \%{ }^{235} \mathrm{U}\right)$.

Referring to Figure $1.1^{2}$, the general enrichment process at a gas centrifuge enrichment plant can be summarized as

1. Feed cylinders with solid, natural uranium hexafluoride $\left(\mathrm{NUF}_{6}\right)$ are transferred from storage to a heating station in the feed area to convert the solid to gas.

2. Gaseous $\mathrm{NUF}_{6}$ is pumped to a pressure-reduction station and then fed into the cascade hall which contains several centrifuge cascades ${ }^{3}$.

3. The gas enters a cascade at the feed stage and material is dispersed to each centrifuge.

\footnotetext{
${ }^{2}$ This figure has been adapted from Uranium Enrichment Plant Characteristics - a Training Manual for the IAEA by J. M. Whitaker [9].

${ }^{3} \mathrm{~A}$ cascade is an arrangement of centrifuges or other separating elements connected by piping to enhance throughput and enrichment.
} 
4. Gas enriched and depleted in ${ }^{235} \mathrm{U}$ leaves the cascade hall and is collected in the withdrawal area.

5. In the withdrawal area, enriched and depleted material is transferred to product and withdrawal cylinders, respectively.

Full feed cylinders enter the plant and full product cylinders leave the plant to be manufactured into reactor fuel pellets. Full tails cylinders are typically left on-site for long term storage [9].

\subsection{IAEA Safeguards at Gas Centrifuge Plants}

IAEA safeguards at uranium enrichment plants call for the timely detection of the

1. Diversion of significant quantities (SQs) of declared material

2. Excess production of low enriched uranium (LEU) from undeclared feed

3. Production of enriched uranium with greater than declared enrichment, specifically highly enriched uranium (HEU) [8]

As related to gas centrifuge enrichment plants (GCEPs), the IAEA definitions for enrichment classification and timeliness goals are defined in Table 1.2 [3]. The term significant quantity was developed by the IAEA to characterize the approximate amount of nuclear material which is necessary to manufacture a nuclear explosive device [3]. The timeliness goals are used to establish the frequency of on-site inspections at a particular nuclear facility [3].

Traditional safeguards approaches for GCEPs were established by the Hexapartite Safeguards Project (HSP) in the early 1980's [10]. The HSP calls for a combination of nuclear material accountancy outside the cascade hall and limited frequency unannounced access (LFUA) inside the cascade hall [11]. This approach allows inspectors to verify the absence of LEU diversion by verifying material flows and inventories through physical 
Table 1.2: IAEA definitions of significant quantities and timeliness goals

\begin{tabular}{lccc}
\hline Material & $\begin{array}{c}\text { Enrichment Level } \\
\left(\%{ }^{235} \mathrm{U}\right)\end{array}$ & $\begin{array}{c}\mathrm{SQ} \\
\left(\mathrm{kg}{ }^{235} \mathrm{U}\right)\end{array}$ & Timeliness Goal \\
\hline Depleted Uranium (DU) & $0.2-0.3$ & 20 ton & 1 year \\
Natural Uranium (NU) & 0.711 & 10 ton & 1 year \\
Low Enriched Uranium (LEU) & $3-5, \leq 20$ & 75 & 1 year \\
High Enriched Uranium (HEU) & $\geq 20$ & 25 & 1 month \\
\hline
\end{tabular}

inventory verification (PIV) and the absence of HEU production by verifying that the cascade is operating as declared through visual inspection $[8,10]$. The frequency of onsite inspections depends on the specific agreement between the IAEA and nation State, but for facilities up to 1 million SWU/year, inspections outside the cascade hall typically occur 12-15 times per year with 3 working days for routine work and 2 weeks for PIV. LFUA inspections can occur 4-12 times per year with visits lasting 1 to 8 hours per day [8]. Additionally, a physical inventory verification (PIV) is performed once per year, taking roughly 3 weeks. This total effort adds up to nearly 100 days of inspection per plant per year.

As shown in Figure 1.2, the number of SQs of nuclear material under IAEA safeguards has grown to over 150,000 in 2007 and is projected to increase to over 400,000 by 2030 . Over that time, the projected safeguards budget per SQ is projected to decrease from roughly $\$ 15,000$ per SQ to under $\$ 300$ per SQ in 2030 [12]. With the increase of world nuclear technology holders [13], in order for the IAEA to continue providing the same level of confidence in verifying the absence of off-normal enrichment operations, there has been a great emphasis in the field to enhance the efficiency and effectiveness of IAEA safeguards. 


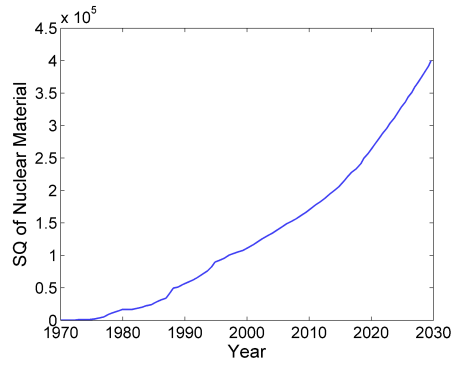

(a)

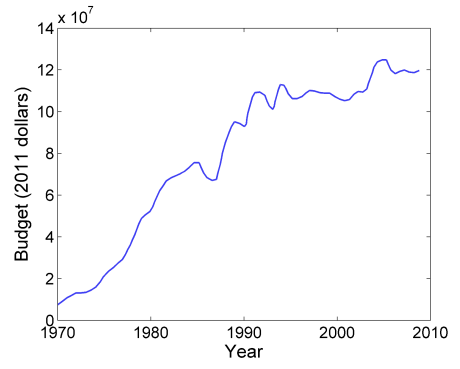

(b)

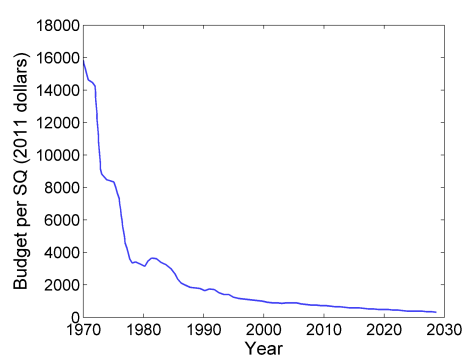

(c)

Figure 1.2: The time evolution of: (a) Significant Quantities (SQs) of nuclear material under IAEA safeguards, (b) the budget of the IAEA, (c) the safeguards budget per SQ. Due to the large increase of nuclear material under safeguards coupled with the stagnant budget of the IAEA, the safeguards budget per SQ has dropped from nearly $\$ 15,000$ per SQ to under $\$ 300$ per SQ. This budgetary constraint has led to an effort to perform more efficient safeguards activities.

\subsubsection{Current Safeguards Technologies}

In an effort to enhance the effectiveness and efficiency of on-site inspections at GCEPs, various safeguards tools have been used or are being developed. Those currently in use include non-destructive assay (NDA) measurements, destructive assay (DA) measurements, and environmental sampling (ES) [8]. NDA detectors rely on gammaray and x-ray fluorescence measurements to determine the enrichment level in GCEP equipment. These detectors are used portably during inspections or have been installed on cascade header piping such as the continuous enrichment monitor (CEMO) [8]. However, these measurements are heavily dependent on equipment geometry, wall thickness, and material buildup on equipment walls. Additionally, the measurement is temperature sensitive and requires extensive calibration [14]. IAEA experience using NDA techniques have shown higher than desired uncertainties in measurements [15]. DA measurements can accurately determine enrichment levels, but can only give information about how the plant is currently operating and is not preferred by the operator $[8,16]$. ES is performed by taking swipe samples of areas where trace amounts of material may exit the cascade, including header pipe connections, sampling stations, and cylinder connections [8]. Samples are 
sent back to the IAEA to be analyzed with mass spectrometry to detect individual micronsize particles. Using this method, IAEA analysts can determine if there are enrichments higher than declared, if there is undeclared feed material, and to a certain extent, changes in operating conditions $[8,17]$. However, it is possible that the measured enrichments may exceed that of the declared values without the GCEP operator attempting to proliferate. This issue will be the focus of a study developed later in this dissertation.

\subsubsection{Next Generation Safeguards Technologies and Approaches}

In an effort to reduce the amount of on-site inspection activities, new, advanced safeguards tools being developed to verify normal operation of a GCEP and to detect diversion and over production of nuclear material. The proposed approaches are designed to complement the current safeguards system and help the IAEA to continue meeting safeguards goals. The so-called next generation of safeguards technologies include flow monitoring $[18,19]$, advanced enrichment monitoring [20-22], cylinder identification and tracking [23], feed and withdrawal process monitoring [24,25], on-site, near-real-time environmental sampling [26], and cylinder assay monitoring [27]. These technologies offer promise for enhancing verification procedures but most rely on cascade external parameters which an operator may be able to change with minimal chance of detection. Additionally, these proposed systems may be costly to implement, may not be implemented in every GCEP, and there are concerns that they may be too intrusive [28].

Of the technologies listed above, a combination of load-cell monitoring of feed and withdrawal cylinders, online enrichment monitoring, and high-accuracy cylinder weight measurements is a preferred method for continuously and independently monitoring the $\mathrm{UF}_{6}$ and ${ }^{235} \mathrm{U}$ mass balance $[29,30]$. Considering this combination of technologies, computational modeling and simulation can offer complementary information. In cases where unattended monitoring is not installed or has failed, a transient simulation of a gas centrifuge cascade can provide information regarding transitions to and from intentional 
off-normal scenarios, such as the time taken to go between the two states and the timeframe for which detectable phenomena remain in the cascade. In the case where the technologies are fully operational, simulation can provide qualitative signatures that can distinguish between an unintentional off-normal scenario, due to such events as centrifuge failure, and intentional events such as material diversion. This type of information may aid in reducing the necessary amount of on-site inspection activities.

\subsubsection{Breakout Scenarios}

In the event that a State decides to withdraw from the NPT, safeguards become obsolete and the concern turns to proliferation of material and the capabilities of a State to produce a weapon. A GCEP designed to produce LEU can be modified to produce HEU in a variety of ways called breakout scenarios [31-33]. Three main strategies exist for acting out a breakout scenario, batch recycling, partial reconfiguration, and cascade interconnection. In the batch recycling case, the cascade is physically unmodified, and product material from the cascade is stored and reintroduced into the cascade to produce a higher enrichment. Partial or total reconfiguration consists of physically changing the shape of the cascade by altering piping. This case is considered unlikely because it would require a great deal

of effort to modify the cascade piping system and connection. Cascade interconnection involves connecting several cascades in series to produce HEU. This is the case that has been historically used to produce weapon grade material [32] and is considered the most plausible.

\subsection{Outline of Dissertation and Objectives}

Based on the current state of the art and future developments of safeguards technologies and the increase in popularity of the gas centrifuge enrichment process, there is a need for modeling tools to provide qualitative and quantitative safeguards-related information. 
As such, the main goal of this work is to develop computational models to simulate the operation of gas centrifuge enrichment cascades and associated proliferation scenarios. Specifically, this research will focus on the verification objectives of the IAEA, i.e. the diversion of declared material with and without the addition of undeclared feed material and the production of higher than declared enrichments. The methods developed in this dissertation will be complementary to the current and future generation of safeguards technologies. One important aspect in the development of these models is that they are intended to be generic and rely on inputs that would be available to an inspector.

The main motivations and objectives of this work can be summarized as:

Motivation: The classified nature of gas centrifuge development limits the details available to properly analyze the separative performance of a centrifuge.

Objective: Develop a method to characterize the performance of a gas centrifuge over a range of operating parameters based on the knowledge of one operational point.

Motivation: In the event a State withdraws from the NPT, it is necessary to understand the capacity of that State to produce weapons-grade uranium.

Objective: Develop an approach to calculate the proliferation capabilities of an enrichment plant and estimate breakout times.

Motivation: Discrepancies between measured enrichment from environmental sampling and expected enrichments.

Objective: Develop a method to predict the level of enrichments that can be expected in a particular cascade. 
Motivation: Anomalies in measured enrichment from unattended online enrichment monitoring and knowledge of time-frames of detectable phenomena associated with proliferation scenarios.

Objective: Develop a transient fluid dynamics and isotope separation model of a gas centrifuge cascade and study normal and off-normal operating modes.

The remaining chapters of this dissertation are organized in the following manner:

- Chapter 2 - The Gas Centrifuge: Internal Flow and Theory

The fundamental theory of fluid flow and isotope separation in a gas centrifuge is detailed. A brief review of the developmental history of the gas centrifuge is provided and a short review of flow field solution methods is discussed. Additionally, illustrative results of the separative performance of two generic centrifuges are presented.

- Chapter 3 - Cascade Theory

The basic theory of cascades is presented for general and ideal cascades. Some simplifications and limiting conditions are also detailed.

- Chapter 4-GCEPs and Proliferation

Gas centrifuge enrichment plant operation is discussed in more detail and proliferation scenarios are defined.

- Chapter 5 - Quantifying Reasonable Enrichment Levels in a Cascade

A method for predicting the full range of concentrations that may be present in a particular cascade due to startup is developed. A generic cascade is studied to show what a discrepancy between normal operation and intentional misuse might look like.

- Chapter 6 - Semi-Empirical Method of Calculating a Centrifuge Performance Map A semi-empirical method for calculating centrifuge performance based on target separation parameters is detailed. The model is verified by comparing separation 
results with the Pancake code at the centrifuge and cascade level. Good agreement is found between the two models.

- Chapter 7 - A Fixed Plant Method for Quantifying Breakout Times

A fixed-plant, general cascade method is developed to determine the breakout time for an enrichment plant operating in a cascade interconnection scenario. The method is used to study the Fuel Enrichment Plant in Natanz, Iran.

- Chapter 8 - Transient Modeling of Gas Centrifuge Cascades

A transient fluid dynamics and isotope separation model of a generic gas centrifuge cascade is developed. The model is used to study the transient operation of a cascade in normal and off-normal operation. A verification case is provided and predicted flow transient agree well.

- Chapter 9 - Summary, Conclusions, and Future Work

The major results of this dissertation are summarized and the relevance of the contributions developed in this work are discussed. 


\section{Chapter 2}

\section{The Gas Centrifuge:}

\section{Internal Flow and Isotope Transport}

\subsection{Overview}

A gas centrifuge (Figure 2.1 [31]) is essentially a hollow rotor that is rapidly spun about its axis. This rotation imparts a strong centrifugal field on the gas contained in the rotor, creating a high stratification of the gas. In the uranium enrichment process, this mechanism is leveraged to separate the isotopes of uranium with the goal of extracting a product that is more concentrated in the desired isotope. In the uranium enrichment process, $\mathrm{UF}_{6}$ is introduced into the centrifuge near the axis (typically near the midpoint of the centrifuge as well) via a feed pipe. Due to the stratification and pressure diffusion, the heavy isotope,

${ }^{238} \mathrm{U}$, tends towards the wall of the centrifuge and the lighter isotope, ${ }^{235} \mathrm{U}$, tends towards the axis, creating a radial concentration gradient.

The idea of isotope separation by centrifuge was first proposed by Lindemann and Aston in 1919, but it wasn't until 1934 that the first successful separation of isotopes by centrifuge was demonstrated by Jesse Beams at the University of Virginia in 1934 $[31,34,35]$. During the Manhattan Project, the gas centrifuge was investigated as a means to produce weapon grade material for a nuclear weapon. The advantage of the gas centrifuge over other methods of enrichment such as gaseous diffusion was a higher separation effect and lower power consumption. However, due to mechanical issues (mostly insufficient 


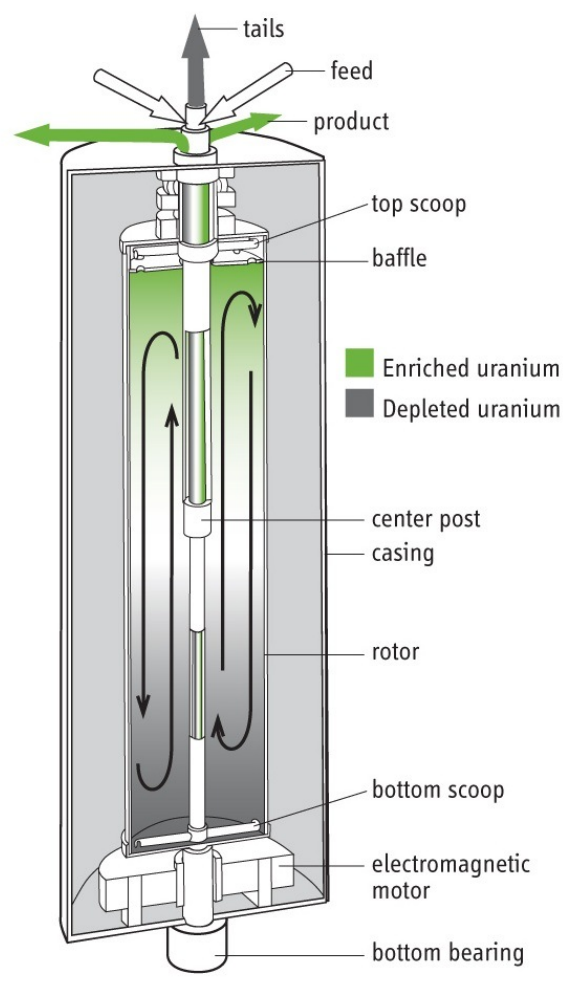

Figure 2.1: A schematic diagram of a countercurrent gas centrifuge. The centrifuge is essentially a hollow cylinder spun rapidly about its axis. This motion induces a rigid body rotation of the gas contained in the cylinder, creating a large pressure gradient between the axis and the wall. The pressure gradient drives a concentration gradient where the lighter isotope tends towards the axis and the heavier isotope towards the wall. This radial separation effect can be enhanced by inducing an axial current.

bearing technology) the program was unsuccessful and terminated in 1943 in favor of gaseous diffusion $[31,35]$. During this time, different types of centrifuges were studied including the evaporative, concurrent, and countercurrent types. It was found that the most preferable method was the countercurrent gas centrifuge because the separation effect could be increased significantly $[34,36]$.

In the countercurrent centrifuge, the radial separation effect is enhanced by perturbing the rigid body rotational flow through a combination of external, mechanical, and thermal drives introducing a convective flow and an axial concentration gradient [37]. The external drive is established through the introduction of material (feed stream) at the axis and withdrawal at the top and bottom of the centrifuge (product and tails stream). The 
mechanical drive can be created by spinning the end caps faster or slower than the periphery of the centrifuge or by keeping the waste scoop open to the separation chamber. The thermal drive is created by establishing a temperature gradient between the top and bottom end caps. These mechanisms drive the axial mass flux in a countercurrent flow pattern, inducing a convective flux that greatly increasing the separation capabilities of the machine [37].

Gas centrifuges can be designed in a variety of aspect ratios and can contain different combinations of mechanical features that help drive the flow and enhance the separative performance. For instance, the Zippe centrifuge (Figure 2.2a [38]) is a subcritical ${ }^{1}$ centrifuge with countercurrent flow driven by the drag caused by the waste scoop near the top of the centrifuge. The flow is protected from the influence of the product scoop by a baffle. The Groth centrifuge (Figure 2.2b [38]) is a supercritical centrifuge with countercurrent flow driven by a maintained axial temperature difference.

\subsubsection{Review of Flow Solution Methods}

Assuming the type of isotope has little effect on the transport properties of the gas, the equations governing the fluid flow and isotope diffusion can be decoupled, with the concentration field solution depending on the flow field [34]. The flow field solutions can be classified [37,39] into three groups: long bowl solutions, boundary layer analyses, and numerical methods. There have been a large number of works related to centrifuge flow modeling. It is not the scope of this dissertation to review all of these works, but a few important models will be introduced and discussed.

Early solution methods (1960's and earlier) relied on the assumption that if the length of the rotor is much larger than the radius, the effects of the end caps would exponentially decay from the ends of the centrifuge and thus a separation of variables approach was taken. This method was independently introduced by Steenbeck (1958) in Germany and

\footnotetext{
${ }^{1}$ A subcritical centrifuge has a length to diameter small enough to avoid flexural modes of vibration.
} 


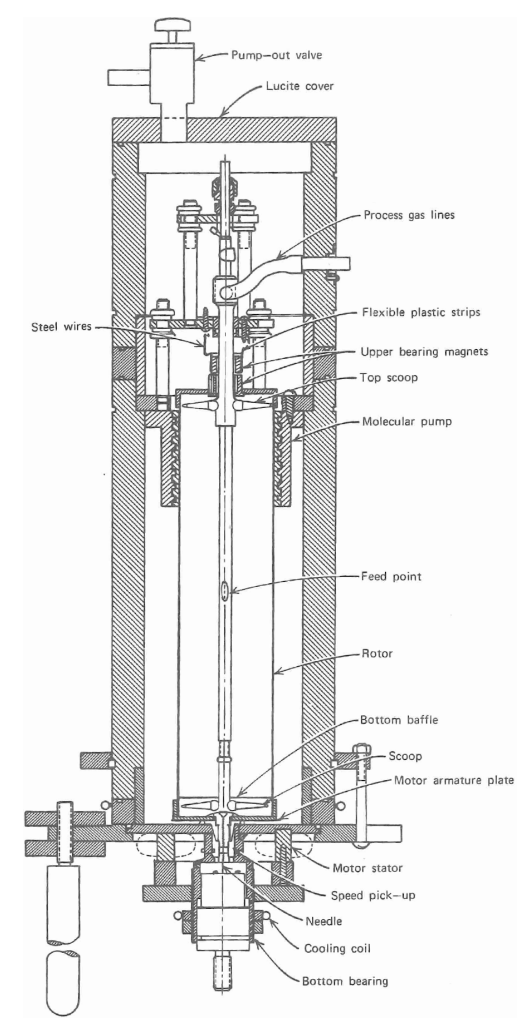

(a) Zippe centrifuge

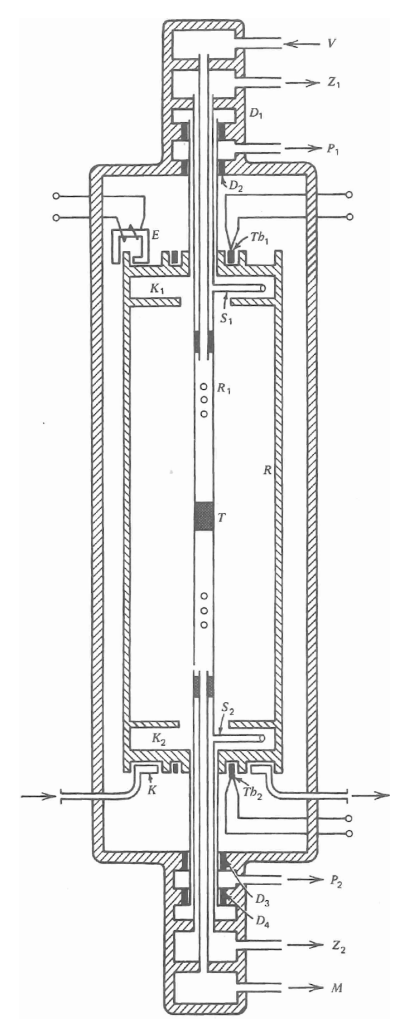

(b) Groth ZG 5 centrifuge

Figure 2.2: Schematic drawings of the (a) Zippe centrifuge and (b) Groth ZG5 centrifuge. The axial countercurrent in the Zippe centrifuge is driven by a thermal gradient between the top and bottom end caps. The flow in the Groth centrifuge is driven by the drag of the waste scoop.

by Parker and Mayo (1963) at the University of Virginia [39]. This method proved to be computationally intensive for the time and other researchers sought methods to simplify the computations. Soubbaramayer (1961) ignored the effects of the endcaps entirely, assuming that the flow field solution only varies in the radial direction [39]. Berman (1963) simplified this further by assuming that the radial and circumferential perturbation velocities were zero [39]. When comparing the axial mass flux in the centrifuge, Olander found good agreement between these models [39]. However, since these models exclude end effects, flow driven by the presence of scoops or mass addition and withdrawal cannot be calculated. A more thorough review of the long bowl solutions can be found in works by Hoglund et. al. [38], Olander [39], and Benedict [40].

The next class of solutions, the boundary layer theory approach, involves separating the 
flow domain into several sub-domains in which certain mechanisms of the flow are more prevalent, typically assuming axisymmetry. This approach was heavily studied from the mid 1960's to mid 1980's and is still considered an important method of solution. Initially, boundary layer theory for rotating flows was studied in the incompressible case and the basic theory of rotating flow in a cylinder was developed by the likes of Stewartson [41], Barcilon and Pedlosky [42], Homsy and Hudson [43,44], and Greenspan [45]. For the gas centrifuge, due to the large radial stratification, incompressible theory is invalid but many of the same principles can be applied. As shown in Figure 2.3a, the flow in the gas centrifuge can be separated into four main flow regions: the inner core, the Ekman boundary layers that form on the top and bottom end caps, the Stewartson boundary layer which forms on the wall, and the extension zones where the Ekman layer and Stewartson layer join ${ }^{2}$.

In the thermally driven gas centrifuge (heated at the bottom), warmer, lighter gas in the bottom Ekman layer is forced towards the axis as cooler heavier gas in the top Ekman layer is forced to the wall. The heavy gas in the top Ekman layer is then forced down the proximity of the wall towards the bottom of the centrifuge and the lighter gas in the bottom Ekman layer is forced up in the inviscid inner core. This circulation flow also creates a circulation region in the viscous Stewartson layer. This is also evident in the axial velocity, gas density, and axial mass flux profiles (Figure 2.3b).

In the literature, two communities have investigated the application of boundary layer theory to the gas centrifuge, the international community and the United States. For the international part, many countries have participated in the development of boundary layer theory for the gas centrifuge including, Japan [46-55], Sweden [56-59], France [60], and The Netherlands [61,62]. Soubbaramayer [37] provides a thorough summary of the international contributions to the application of boundary layer theory to gas centrifuge flow. Efforts by Swedish researchers led to the formation of an international conference dedicated to the field of rotating flows entitled the Workshop on Gases in Strong Rotation

\footnotetext{
${ }^{2}$ Not shown is the division in the Stewartson layer which is necessary for symmetric flow (driven by differentially rotating end caps) [37].
} 


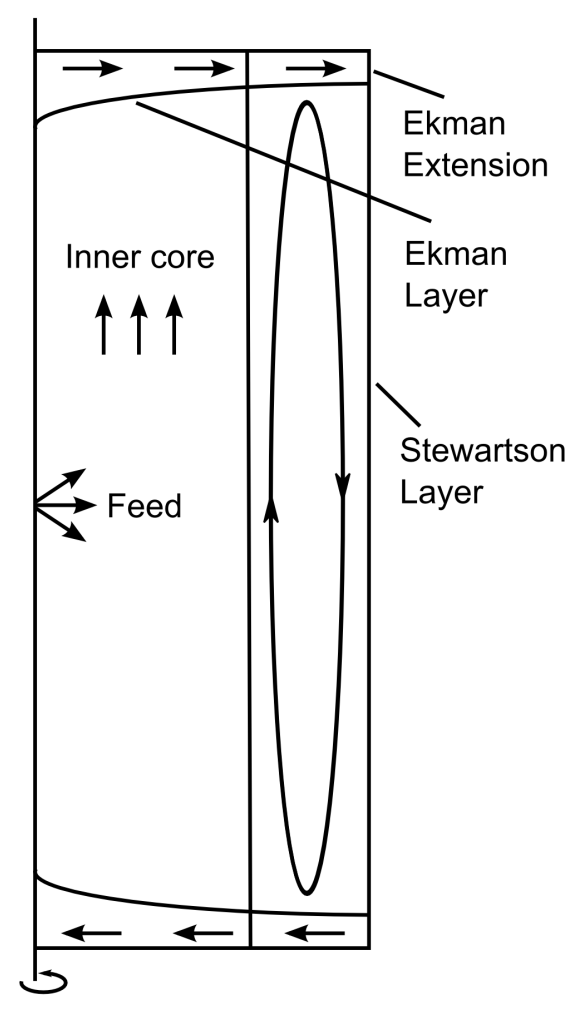

(a) Boundary layers and flow schematic
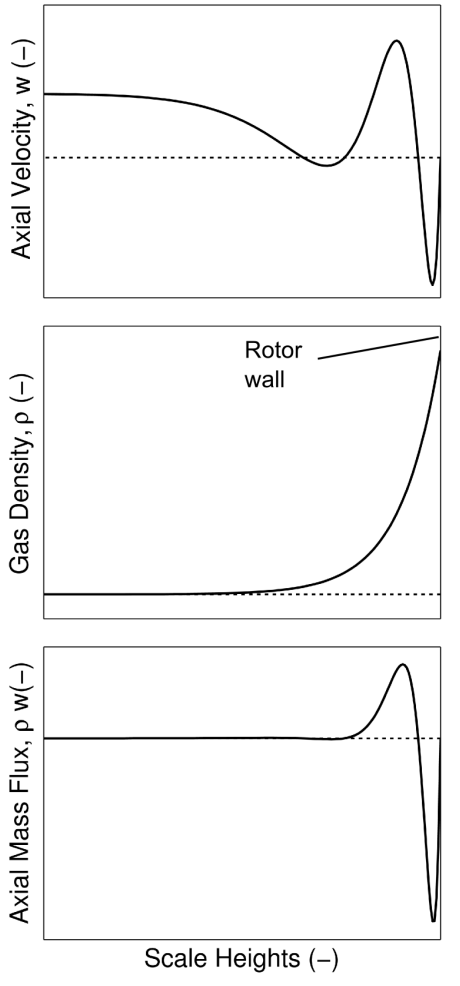

(b) Axial velocity, gas density, and axial mass flux profiles (arb. units)

Figure 2.3: A typical flow diagram in a thermally driven gas centrifuge. Boundary layers form on the top and bottom endcaps (Ekman layers) and the side wall (Stewartson layer). In the top Ekman layer, colder, denser fluid particle are driven to the outside wall and down the centrifuge wall. In the bottom Ekman layer, warmer, lighter fluid particles are driven towards the axis and rise up the centrifuge in the inner, inviscid core. This flow is evident in the axial velocity and mass flux profiles.

beginning in 1975 [63]. This conference is now know as the Workshop on Separation Phenomena in Liquids and Gases and details the current state of the art in the field of separation science.

The United States effort on boundary layer theory in gas centrifuge began in 1961 by a group formed by the United States Atomic Energy Commission led by Lars Onsager [64]. Due to the classified nature of the centrifuge flow group project, this work was not known to the international community at the time and there were some differences in the approaches taken to the problem. The efforts of the group led to a boundary layer method solution for the flow in a gas centrifuge that does not require some of the flow domains that are 
necessary in the models developed by the international community. The details of the Onsager Pancake model with Carrier-Maslen boundary conditions and source-sink flow was first presented by Wood and Morton [64] and will be summarized in Section 2.3.

The third class of flow field solutions involves numerically solving the linearized and nonlinear governing equations. Due to the heavy computational requirements for such a solution, these methods did not begin to appear until 1975. Soubbaramayer [37] provides an excellent summary of the early numerical work in the field. Recent numerical work includes the finite volume approach of Omnes [65] and the nonlinear streamfunction approach of Van Ommen [66]. More advanced work includes exploring transient phenomena in the gas centrifuge [67-69] and the coupling of Direct Simulation Monte Carlo in the rarefied region of the centrifuge [70].

In this chapter, the basic theory of the countercurrent gas centrifuge is discussed. In light of the above discussion, two flow field solution methods will be introduced, the Pancake model (part of the boundary layer theory class) and the pure-axial flow model (part of the long bowl solution class). The theory developed in this chapter follows closely the work of many important reviews on gas centrifuge theory, including works by Cohen [34], Soubbaramayer [37], Olander [39], Wood [64], and Von Halle [71].

\subsection{Governing Equations}

For the cylindrical coordinate system $(r, \theta, z)$, let $(\hat{u}, \hat{v}, \hat{w})$ be the corresponding velocity components with the $\mathrm{z}$ axis along the axis of a right circular cylinder rotating at speed $\Omega$. Assuming axisymmetry, the complete set of governing equations of fluid dynamics in cylindrical coordinate system $\operatorname{are}^{3}[37,72]$

\footnotetext{
${ }^{3}$ Note that the effects of gravity are not included in the axial momentum equation. This is due to the fact that the countercurrent flow is not driven by the gravitational body force, but by the compression-expansion work term in the energy equation due to large radial stratification. The benefit of neglecting this term is that the solution becomes invariant, i.e. it doesn't matter if the centrifuge is heated/cooled on the top or bottom. This has been confirmed in experiments by Groth [73].
} 


$$
\frac{\partial}{\partial z}[\hat{\rho} \hat{w}]+\frac{1}{r} \frac{\partial}{\partial r}[r \hat{\rho} \hat{u}]=0
$$

$$
\hat{\rho}\left[\hat{w} \frac{\partial \hat{u}}{\partial z}+\hat{u} \frac{\partial \hat{u}}{\partial r}-\frac{\hat{v}^{2}}{r}\right]+\frac{\partial \hat{p}}{\partial r}-\mu\left[\left(\hat{\nabla}^{2}-\frac{1}{r^{2}}\right) \hat{u}+\frac{1}{3} \frac{\partial}{\partial r}(\hat{\nabla} \cdot \hat{\mathbf{V}})\right]=0
$$

$$
\hat{\rho}\left[\hat{w} \frac{\partial \hat{v}}{\partial z}+\hat{u} \frac{\partial \hat{v}}{\partial r}+\frac{\hat{u} \hat{v}}{r}\right]-\mu\left(\hat{\nabla}^{2}-\frac{1}{r^{2}}\right) \hat{v}=0
$$

$$
\hat{\rho}\left[\hat{w} \frac{\partial \hat{w}}{\partial z}+\hat{u} \frac{\partial \hat{w}}{\partial r}\right]+\frac{\partial \hat{p}}{\partial z}-\mu\left[\hat{\nabla}^{2} \hat{w}+\frac{1}{3} \frac{\partial}{\partial z}(\hat{\nabla} \cdot \hat{\mathbf{V}})\right]=0
$$

$$
\hat{\rho} c_{v}\left[\hat{w} \frac{\partial \hat{T}}{\partial z}+\hat{u} \frac{\partial \hat{T}}{\partial r}\right]+\hat{p}(\hat{\nabla} \cdot \hat{\mathbf{V}})-\kappa \hat{\nabla}^{2} \hat{T}=0
$$

$$
\hat{p}=\hat{\rho} R_{g} \hat{T}
$$

These equations represent conservation of mass, radial momentum, circumferential momentum, axial momentum, energy ${ }^{4}$ and the state equation. The divergence and Laplacian in cylindrical coordinates are defined as

$$
\begin{aligned}
& (\hat{\nabla} \cdot \hat{\mathbf{V}})=\frac{\partial \hat{w}}{\partial z}+\frac{1}{r} \frac{\partial}{\partial r}(r \hat{u}) \\
& \hat{\nabla}^{2}=\frac{\partial^{2}}{\partial z^{2}}+\frac{1}{r} \frac{\partial}{\partial r}\left(r \frac{\partial}{\partial r}\right)
\end{aligned}
$$

\footnotetext{
${ }^{4}$ It should be noted that viscous dissipation has not been included because these terms will drop out in the perturbation analysis.
} 


\subsubsection{Linearization Process}

Because these full set of equations are nonlinear and complex, exact solutions are not tractable and thus approximations must be made. One simplification is to assume that the flow can be separated into a base state, rigid body rotational flow and a linearly perturbed flow. This can be written for the primitive flow variables as

$$
\hat{\xi}=\bar{\xi}+\varepsilon \xi^{\prime}
$$

where $\hat{\xi}$ represents the primitive flow variables $\{\hat{u}, \hat{v}, \hat{w}, \hat{p}, \hat{\rho}, \hat{T}\}, \bar{\xi}$ represents the zeroth order terms of the primitive flow variables, $\xi^{\prime}$ represents the perturbed state of the flow, and $\varepsilon$ is the Rossby number which measures the magnitude of the perturbation. The definition of the Rossby number depends on the type of flow perturbation. For example, the Rossby number for a wall thermal drive is defined as

$$
\varepsilon=\frac{\Delta T}{2 T_{0}}
$$

where $\Delta T$ is the temperature difference between the bottom and top of the centrifuge and $T_{0}$ is the average temperature of the gas.

Substituting $\hat{\xi}$ into Equations (2.1) - (2.6) and collecting terms of order $\varepsilon^{0}$ and $\varepsilon^{1}$ yields the zeroth order and first order governing equations.

In the absence of perturbations to the flow (i.e. disturbances such as temperature variation, scoop drag, and introduction and withdrawal of mass), the fluid will rotate as an isothermal rigid body for which the base velocity components are

$$
\bar{u}=0, \quad \bar{v}=\Omega r, \quad \bar{w}=0, \quad \bar{T}=T_{0}
$$

Substituting Equations (2.11) into the zeroth order form of Equations (2.1) - (2.5) yields a force balance between the centrifugal and pressure forces 


$$
\frac{d \bar{p}}{d r}=\bar{\rho} r \Omega^{2}
$$

Substituting the ideal gas law, Equation (2.6), into Equation (2.12), the base state pressure distribution in the centrifuge is found to be

$$
\bar{p}=p_{w} \exp \left\{A^{2}\left[\left(\frac{r}{a}\right)^{2}-1\right]\right\}
$$

where $p_{w}$ is the pressure of gas at the wall, $a$ is the radius of the cylinder, and $A^{2}$ is the stratification parameter defined as

$$
A^{2}=\frac{(\Omega a)^{2}}{2 R_{g} T_{0}}
$$

The stratification parameter defines the number of scale heights (e-folding heights) in the centrifuge. The zeroth order equation of state becomes

$$
\bar{p}=\bar{\rho} R_{g} T_{0}
$$

The first order governing equations are then

$$
\begin{gathered}
\frac{\partial}{\partial z}\left[\bar{\rho} w^{\prime}\right]+\frac{1}{r} \frac{\partial}{\partial r}\left[r \bar{\rho} u^{\prime}\right]=0 \\
-r \Omega^{2} \rho^{\prime}-2 \Omega \bar{\rho} v^{\prime}+\frac{\partial p^{\prime}}{\partial r}-\mu\left\{\frac{\partial^{2} u^{\prime}}{\partial z^{2}}+\frac{4}{3}\left[\frac{1}{r} \frac{\partial}{\partial r}\left(r \frac{\partial u^{\prime}}{\partial r}\right)-\frac{u^{\prime}}{r^{2}}\right]+\frac{1}{3} \frac{\partial^{2} w^{\prime}}{\partial r \partial z}\right\}=0 \\
2 \Omega \bar{\rho} u^{\prime}-\mu\left\{\frac{\partial^{2} v^{\prime}}{\partial z^{2}}+\frac{1}{r} \frac{\partial}{\partial r}\left(r \frac{\partial v^{\prime}}{\partial r}\right)-\frac{v^{\prime}}{r^{2}}\right\}=0 \\
\frac{\partial p^{\prime}}{\partial z}-\mu\left\{\frac{4}{3} \frac{\partial^{2} w^{\prime}}{\partial z^{2}}+\frac{1}{r} \frac{\partial}{\partial r}\left(r \frac{\partial w^{\prime}}{\partial r}\right)+\frac{1}{3} \frac{1}{r} \frac{\partial^{2}}{\partial z \partial r}\left(r u^{\prime}\right)\right\}=0
\end{gathered}
$$




$$
\begin{gathered}
\bar{p}\left[\frac{\partial w^{\prime}}{\partial z}+\frac{1}{r} \frac{\partial}{\partial r}\left(r u^{\prime}\right)\right]-\kappa\left[\frac{\partial^{2} T^{\prime}}{\partial z^{2}}+\frac{1}{r} \frac{\partial}{\partial r}\left(r \frac{\partial T^{\prime}}{\partial r}\right)\right]=0 \\
p^{\prime}=\bar{\rho} R_{g} T^{\prime}+\rho^{\prime} R_{g} \bar{T}
\end{gathered}
$$

Equations (2.17), (2.19), and (2.20) can be further manipulated, giving

$$
\begin{gathered}
-r \Omega^{2} \rho^{\prime}-2 \Omega \bar{\rho} v^{\prime}+\frac{\partial p^{\prime}}{\partial r}-\mu\left\{\hat{\nabla}^{2} u^{\prime}-\frac{u^{\prime}}{r^{2}}-\frac{\Omega^{2}}{3 R_{g} T_{0}} \frac{\partial}{\partial r}\left(r u^{\prime}\right)\right\}=0 \\
\frac{\partial p^{\prime}}{\partial z}-\mu\left\{\hat{\nabla}^{2} w^{\prime}-\frac{\Omega^{2} r}{3 R_{g} T_{0}} \frac{\partial u^{\prime}}{\partial z}\right\}=0 \\
r \Omega^{2} \bar{\rho} u^{\prime}+\kappa\left[\frac{\partial^{2} T^{\prime}}{\partial z^{2}}+\frac{1}{r} \frac{\partial}{\partial r}\left(r \frac{\partial T^{\prime}}{\partial r}\right)\right]=0
\end{gathered}
$$

Equations (2.16), (2.18), (2.21), (2.22), (2.23), and (2.24) are now the full set of linearized governing equations.

\subsubsection{Non-Dimensionalization}

It is often useful to nondimensionalize the set of linearized governing equations and so the following terms are defined

$$
\begin{gathered}
\eta=\frac{r}{a}, \quad y=\frac{z}{a}, \quad \rho_{0}=\frac{\bar{\rho}}{\rho_{w}}, \quad \operatorname{Re}=\frac{\rho_{w} \Omega a^{2}}{\mu} \\
u=\frac{u^{\prime}}{\Omega a}, \quad w=\frac{w^{\prime}}{\Omega a}, \quad v=\frac{v^{\prime}}{\Omega r}, \quad \operatorname{Pr}=\frac{c_{p} \mu}{\kappa} \\
T=\frac{T^{\prime}}{T_{0}}, \quad \rho=\frac{\rho^{\prime}}{\rho_{w}}, \quad p=\frac{p^{\prime}}{p_{w}}, \quad \mathrm{~S}=1+\frac{\operatorname{Pr} A^{2}(\gamma-1)}{2 \gamma}
\end{gathered}
$$


where $\rho_{w}$ is the gas density at the wall of the centrifuge. Substituting Equations (2.25) into the set of linearized governing equations yields

$$
\begin{gathered}
\frac{\partial}{\partial \eta}\left(\eta \rho_{0} u\right)+\eta \rho_{0} \frac{\partial w}{\partial y}=0 \\
-\eta \rho-2 \eta \rho_{0} v+\frac{1}{2 A^{2}} \frac{\partial p}{\partial \eta}-\frac{1}{\operatorname{Re}}\left\{\nabla^{2} u-\frac{u}{\eta^{2}}-\frac{2 A^{2}}{3} \frac{\partial}{\partial \eta}(\eta u)\right\}=0 \\
2 \rho_{0} u-\frac{1}{\operatorname{Re}}\left[\nabla^{2}(\eta v)-\frac{v}{\eta}\right]=0 \\
\frac{\partial p}{\partial y}-\frac{2 A^{2}}{\operatorname{Re}}\left[\nabla^{2} w-\frac{2 A^{2}}{3} \eta \frac{\partial u}{\partial y}\right]=0 \\
4 \operatorname{Re}(S-1) \eta \rho_{0} u+\nabla^{2} T=0 \\
p=\rho+\rho_{0} T
\end{gathered}
$$

where $\nabla^{2}=a^{2} \hat{\nabla}^{2}$.

\subsection{Onsager's Model}

Similar to the other boundary layer approaches, the Onsager model separates the flow into two regions, the internal flow region and the Ekman layers on the top and bottom of the centrifuge. Referring back to Figure 2.3a, in the Onsager model, the internal flow region covers the Stewartson layer and the inner core region. In the internal flow region, axial diffusion is negligible compared to convective flow and terms associated with axial diffusion of momentum and energy are ignored. In the Ekman boundary layers, axial diffusion plays an important role and can have a significant effect on the internal flow. 
It will be shown that the effect of the Ekman layer on the internal flow can be applied as a boundary condition to the governing equations of the internal flow region.

For the internal flow region, the Onsager model [64] is derived by making the following assumptions:

1. Neglect terms involving axial diffusion of heat and momentum.

2. Retain only the viscous terms that are most highly differentiated in the radial direction.

3. For $A^{2} \gg 1$, most of the gas is retained very close to the wall of the centrifuge and curvature may be neglected by setting $\eta=1$ where it appears algebraically. This assumption is called the 'pancake' approximation and is not necessary.

Introducing a new radial variable which measures the distance from the rotor wall in scale heights (e-folding heights)

$$
x=A^{2}\left(1-\eta^{2}\right)
$$

and including source terms, the Onsager model for the fluid dynamics in the internal region of the centrifuge is represented by the following system of equations

$$
\begin{gathered}
e^{-x} \frac{\partial w}{\partial y}-2 A^{2} \frac{\partial}{\partial x}\left[e^{-x} u\right]=\mathscr{M} \\
\phi-\frac{\partial}{\partial x}\left[e^{x} p\right]=e^{x} \mathscr{U} \\
\frac{\partial^{2} \phi}{\partial x^{2}}+\frac{\operatorname{Re} S}{A^{4}} e^{-x} u=2 \mathscr{V}-\mathscr{T} \\
\frac{\partial p}{\partial y}-\frac{8 A^{6}}{\operatorname{Re}} \frac{\partial^{2} w}{\partial x^{2}}=\mathscr{W}
\end{gathered}
$$




$$
-4 A^{4} \frac{\partial^{2} h}{\partial x^{2}}-\frac{\partial^{2} h}{\partial y^{2}}=\mathscr{T}+2(S-1) \mathscr{V}
$$

where $\phi=T-2 v$ and $h=T+2(S-1) v$. Here, $\mathscr{M}, \mathscr{U}, \mathscr{V}, \mathscr{W}, \mathscr{T}$ are sources/sinks of mass, radial momentum, angular momentum, axial momentum, and energy. These terms can be used to account for the presence of a scoop and the addition and withdrawal of gas.

Following Wood and Morton [64], these equations can be manipulated to form a single partial differential equation in terms of a master potential function

$$
\frac{\partial^{2}}{\partial x^{2}}\left[e^{x} \frac{\partial^{2}}{\partial x^{2}}\left(e^{x} \frac{\partial^{2} \chi}{\partial x^{2}}\right)\right]+B^{2} \frac{\partial^{2} \chi}{\partial y^{2}}=F(x, y)
$$

where

$$
\begin{aligned}
F(x, y)=\frac{B^{2} A^{2}}{2 \operatorname{Re} S} \int_{x}^{x_{T}}\left[\frac{\partial \mathscr{T}}{\partial y}-2 \frac{\partial \mathscr{V}}{\partial y}\right] d x^{\prime} & -\frac{B^{2}}{4 A^{4}} \int_{x}^{x_{T}} \int_{0}^{x^{\prime}} \frac{\partial \mathscr{M}}{\partial y} d x^{\prime} d x^{\prime \prime} \\
& -\frac{B^{2} A^{2}}{2 \operatorname{Re} S}\left[\frac{\partial}{\partial x}\left(e^{x} \frac{\partial \mathscr{U}}{\partial y}\right)+\frac{\partial^{2}}{\partial x^{2}}\left(e^{x} \mathscr{W}\right)\right]
\end{aligned}
$$

and

$$
B=\frac{\operatorname{Re} S^{1 / 2}}{4 A^{6}}
$$

Equation (2.38) is a sixth-order, linear, partial differential equation requiring six radial boundary conditions and two axial boundary conditions. The six radial boundary conditions and mathematical representations are given in Table 2.1. In the near wall region of the top and bottom of the centrifuge, Ekman boundary layers develop. In these regions, the Onsager model is not valid because axial diffusion plays an important role in transport. In lieu of retaining these terms in the linearized governing equations and solving for the flow in the Ekman layers, Carrier and Maslen [74,75] developed a boundary condition for the Pancake equation that when imposed, has the same effect on the internal flow as does 
resolving the Ekman layer flow. The axial boundary conditions are found to be

$$
\begin{aligned}
& B^{2} \frac{\partial \chi}{\partial y}(x, 0)=-2 A B^{3 / 2} \frac{\partial}{\partial x}\left[e^{x / 2} \frac{\partial \chi}{\partial x}(x, 0)\right]+g_{0}(x) \\
& B^{2} \frac{\partial \chi}{\partial y}\left(x, y_{T}\right)=2 A B^{3 / 2} \frac{\partial}{\partial x}\left[e^{x / 2} \frac{\partial \chi}{\partial x}\left(x, y_{T}\right)\right]+g_{1}(x)
\end{aligned}
$$

where

$$
\begin{gathered}
g_{0}(x)=\frac{B^{3 / 2}}{2 \pi A} \frac{d}{d x}\left[e^{x / 2} \dot{m}_{0}(x)\right]-\frac{B}{8 A^{4} S^{1 / 2}} \frac{d \bar{\phi}_{0}}{d x}(x) \\
\dot{m}_{0}=\frac{\pi}{A^{2}} \int_{0}^{x} \rho_{0} \bar{w}_{0}\left(x^{\prime}\right) d x^{\prime} \\
g_{1}(x)=-\frac{B^{3 / 2}}{2 \pi A} \frac{d}{d x}\left[e^{x / 2} \dot{m}_{1}(x)\right]-\frac{B}{8 A^{4} S^{1 / 2}} \frac{d \bar{\phi}_{1}}{d x}(x) \\
\dot{m}_{1}=\frac{\pi}{A^{2}} \int_{0}^{x} \rho_{0} \bar{w}_{1}\left(x^{\prime}\right) d x^{\prime}
\end{gathered}
$$

Here $\bar{\phi}_{0}, \bar{\phi}_{1}$ are prescribed functions on the bottom and top end cap respectively (combination of temperature and circumferential velocity perturbation) and $\hat{w}_{0}, \hat{w}_{1}$ are the prescribed axial velocity perturbations at the bottom and top end caps.

There have been many works that have focused on solving the Pancake equation. Wood and Morton [64] solved the homogenous Onsager model using separation of variables and eigenfunction expansion. Wood and Sanders [76] extended this solution to include the effects of source and sink terms. Gunzburger and Wood [77] solved the nonhomogenous Onsager model using a finite element method. Ribando [78] and more recently DeStadler [79] solved the homogeneous Onsager equations using a finite difference method. 
Table 2.1: Radial boundary conditions for the Onsager Pancake equation.

\begin{tabular}{|c|c|c|}
\hline Boundary Condition & Mathematical Statement & \\
\hline no axial velocity at rotor wall & $\frac{\partial \chi}{\partial x}(0, y)=0$ & \\
\hline no radial velocity at rotor wall & $\frac{\partial^{2} \chi}{\partial x^{2}}(0, y)=0$ & \\
\hline temperature gradient on rotor wall & $\frac{\partial^{2}}{\partial x^{2}}\left[e^{x} \frac{\partial^{2}}{\partial x^{2}}\left(e^{x} \frac{\partial^{2}}{\partial x^{2}} \chi(0, y)\right)\right.$ & $=\frac{\operatorname{Re}}{32 A^{10}} \frac{\partial f}{\partial y}$ \\
\hline $\begin{array}{l}\text { no radial velocity at the top of the } \\
\text { atmosphere }\end{array}$ & $\frac{\partial \chi}{\partial y}\left(x_{T}, y\right)=0$ & \\
\hline $\begin{array}{l}\text { no radial gradient of axial velocity at } \\
\text { top of atmosphere }\end{array}$ & $\frac{\partial \chi}{\partial x}\left(x_{T}, y\right)=0$ & \\
\hline $\begin{array}{l}\text { no radial gradient of temperature and } \\
\text { circumferential velocity at the top of } \\
\text { atmosphere }\end{array}$ & $\frac{\partial}{\partial x}\left[e^{x} \frac{\partial^{2} \chi}{\partial x^{2}}\right]=0$ & \\
\hline
\end{tabular}

\subsection{The Pure-Axial Flow Model}

The pure-axial flow or rod-like flow model $[71,80]$ is included in the class of solutions deemed the long bowl solutions because of the assumption that if the length of the centrifuge is much larger than the radius, a large portion of the velocity field will be independent of end effects and thus the axial component. These types of models cannot take into effect flow drives such as scoop drag, differentially rotating end caps, or sources and sinks of mass. The pure-axial flow model an be found two ways, by assuming $u, v, w$ are independent of $z$, or by assuming $u=v=0$.

Substituting $u=v=0$ into the Equation (2.26) gives

$$
\frac{\partial w}{\partial y}=0 \rightarrow w=w(\eta)
$$


thus the axial velocity is only a function of the radial direction. The other nondimensional linearized governing equations (see Section 2.2.2) reduce to

$$
\begin{gathered}
\frac{\partial p}{\partial \eta}=2 A^{2} \eta \rho \\
\frac{\partial p}{\partial y}=\frac{2 A^{2}}{\operatorname{Re}}\left[\frac{1}{\eta} \frac{\partial}{\partial \eta}\left(\eta \frac{\partial w}{\partial \eta}\right)\right] \\
\frac{\partial^{2} T}{\partial y^{2}}+\frac{1}{\eta} \frac{\partial}{\partial \eta}\left[\eta \frac{\partial T}{\partial \eta}\right]=0
\end{gathered}
$$

Taking $\frac{\partial}{\partial y}$ of Equation (2.48) and $\frac{\partial}{\partial \eta}$ of Equation (2.49) and combining yields

$$
\eta \frac{\partial \rho}{\partial y}=\frac{1}{\operatorname{Re}} \frac{\partial}{\partial \eta}\left[\frac{1}{\eta} \frac{\partial}{\partial \eta}\left(\eta \frac{\partial w}{\partial \eta}\right)\right]
$$

The perturbation density can be removed by substituting Equation (2.31) into Equation (2.49) and substituting this result into Equation (2.51) to get

$$
2 A^{2} \eta\left[\frac{1}{\eta} \frac{\partial}{\partial \eta}\left(\eta \frac{\partial w}{\partial \eta}\right)\right]-\operatorname{Re} \rho_{0} \eta \frac{\partial T}{\partial y}=\frac{d}{d \eta}\left[\frac{1}{\eta} \frac{\partial}{\partial \eta}\left(\eta \frac{\partial w}{\partial \eta}\right)\right]
$$

Changing to the scale height coordinate, Equations (2.52) and (2.50) become

$$
\begin{aligned}
\frac{d^{2}}{d x^{2}}\left[\left(1-\frac{x}{A^{2}}\right) \frac{d w}{d x}\right]+\frac{d}{d x}\left[\left(1-\frac{x}{A^{2}}\right) \frac{d w}{d x}\right] & =\frac{\operatorname{Re}}{8 A^{2}} e^{-x} \frac{\partial T}{\partial y} \\
\frac{\partial^{2} T}{\partial y^{2}}+4 A^{2} \frac{\partial}{\partial x}\left[\left(1-\frac{x}{A^{2}}\right) \frac{\partial T}{\partial x}\right] & =0
\end{aligned}
$$

Here Von Halle [71] makes the thin film approximation, i.e. $\left[1-\frac{x}{A^{2}}\right] \approx 1$. Following Hu et. al. [80], this restriction will not be made. Examining Equation (2.53), it can be seen that $\frac{\partial T}{\partial y}=f(x)$, thus $\frac{\partial^{2} T}{\partial y^{2}}=0$ and Equation (2.54) becomes 


$$
\frac{\partial}{\partial x}\left[\left(1-\frac{x}{A^{2}}\right) \frac{\partial T}{\partial x}\right]=0
$$

which can be integrated to find

$$
T(x, y)=a_{1}(y)-a_{2}(y) A^{2} \ln \left(A^{2}-x\right)
$$

The temperature field must be finite at the axis $\left(x=A^{2}\right)$, so $a_{2}(y) \rightarrow 0$, giving $T(y)=$ $a_{1}(y)$. Let $a_{1}(y)=\lambda y+\kappa$, then Equation (2.53) becomes

$$
\frac{d^{2}}{d x^{2}}\left[\left(1-\frac{x}{A^{2}}\right) \frac{d w}{d x}\right]+\frac{d}{d x}\left[\left(1-\frac{x}{A^{2}}\right) \frac{d w}{d x}\right]=\zeta e^{-x}
$$

where $\zeta=\frac{\lambda \operatorname{Re}}{8 A^{6}}$. The boundary conditions for this third order, nonhomogeneous, ordinary differential equation are

$$
\begin{array}{cc}
w(0)=0 & \text { no-slip condition } \\
\left.\frac{d w}{d x}\right|_{x=\varepsilon A^{2}} & \text { symmetry at axis } \\
\frac{P^{*} A^{2}}{\pi \rho_{w} \Omega a^{3}}=Q=\int_{0}^{\varepsilon A^{2}} w e^{-x} d x & \text { net axial flux }
\end{array}
$$

where $P^{*}=L_{c}$ in the enriching section of the centrifuge and $P^{*}=-D_{c}$ in the stripping section of the centrifuge. Here, $L_{c}$ is the centrifuge product rate and $D_{c}$ is the centrifuge tails rate. By the change of variable, $w^{*}=\left(1-\frac{x}{A^{2}}\right) \frac{d w}{d x}$, Equation (2.57) can be solved to find

$$
w=-\zeta A^{2} e^{-x}+c_{1} \operatorname{Ei}\left[A^{2}\left(1-\frac{x}{A^{2}}\right)\right]+c_{2} \ln \left[1-\frac{x}{A^{2}}\right]+c_{3}
$$

where 


$$
\operatorname{Ei}(x)=-\int_{-x}^{\infty} \frac{e^{-x}}{x} d x
$$

and the coefficients $c_{1}, c_{2}, c_{3}$ are determined by applying the boundary conditions and can be found in Appendix A.

\subsection{Isotope Transport}

In order to determine the separative performance of a gas centrifuge, the concentration gradient solution must be developed. In this section, the diffusion equation for the gas centrifuge is developed and then simplified using the radial-averaging approach of Cohen $[34,37]$.

Considering a gas mixture of two components, the conservation equation of the desired isotope at steady-state (taking convective and diffusive transport into account) is

$$
\frac{1}{r} \frac{\partial}{\partial r}\left[r \hat{\rho} \hat{u} N+r J_{r}\right]+\frac{\partial}{\partial z}\left[\hat{\rho} \hat{w} N+J_{z}\right]=0
$$

where $N$ is the local concentration of the desired isotope and $J_{r}$ and $J_{z}$ are the radial and axial components of the diffusive flux and are defined as

$$
\begin{gathered}
J_{r}=-\hat{\rho} D\left[\frac{\Delta M \Omega^{2} r}{R_{u} T_{0}} N(1-N)+\frac{\partial N}{\partial r}\right] \\
J_{z}=-\hat{\rho} D \frac{\partial N}{\partial z}
\end{gathered}
$$

Here, $D$ is the self-diffusion coefficient and based on kinetic theory, the product $\hat{\rho} D$ is assumed constant. Recalling the overall conservation of mass equation (Equation (2.1)) and combining with continuity equation of the desired isotope (Equation (2.61)), the governing equation for the axisymmetric, steady-state concentration field is 


$$
\hat{\rho} D \frac{\partial^{2} N}{\partial z^{2}}-\hat{\rho} \hat{w} \frac{\partial N}{\partial z}+\hat{\rho} D \frac{1}{r} \frac{\partial}{\partial r}\left[r \frac{\partial N}{\partial r}+\frac{\Delta M \Omega^{2}}{R_{u} T_{0}} r^{2} N(1-N)\right]+\hat{\rho} \hat{u} \frac{\partial N}{\partial r}=0
$$

Equation (2.64) is a nonlinear partial differential equation which can be solved through a radial averaging procedure which reduces the partial differential equation to an ordinary differential equation for the radial averaged concentration along the length of the centrifuge [37].

\subsubsection{Deriving the Gradient Equation Through Radial Averaging}

The derivation of the radial averaged gradient equation follows that of Von Halle [81]. The goal is to simplify the diffusion equation through a similar method used by Furry, Jones, and Onsager for thermal diffusion columns [82]. The first step is to develop an expression for the net axial transport of the desired component by integrating the axial current over the cross section of the centrifuge

$$
\tau=\int_{0}^{a} 2 \pi r \hat{\rho} \hat{w} N d r-\int_{0}^{a} 2 \pi r \hat{\rho} D \frac{\partial N}{\partial z} d r
$$

where, $\tau=L_{c} N_{c, P}$, in the enriching section of the centrifuge and $\tau=-D_{c} N_{c, W}$ in the stripping section. Here, $N_{c, P}$ is the concentration of the key isotope in the product stream, and $N_{c, W}$ is the concentration in the tails stream. Next, define a flow function of the net axial transport of the gas between radius, $r$, and the rotor wall

$$
\mathscr{G}(r, z)=\int_{r}^{a} 2 \pi \hat{\rho} \hat{w} r d r
$$

where 


$$
\begin{aligned}
\mathscr{G}(0, z) & =\tau^{*} \\
\mathscr{G}(a, z) & =0 \\
\frac{\partial \mathscr{G}}{\partial r} & =-2 \pi r \hat{\rho} \hat{w}
\end{aligned}
$$

Here, $\tau^{*}=L_{c}$ in the enriching section of the centrifuge and $\tau^{*}=-D_{c}$ in the stripping section. Next, rewrite Equation (2.65) in terms of the flow function

$$
\tau=-\int_{0}^{a} \frac{\partial \mathscr{G}}{\partial r} N d r-\int_{0}^{a} 2 \pi r \hat{\rho} D \frac{\partial N}{\partial z} d r
$$

and integrate the first term by parts to find

$$
\tau=\tau^{*} N(0, z)+\int_{0}^{a} \mathscr{G} \frac{\partial N}{\partial r} d r-\int_{0}^{a} 2 \pi r \hat{\rho} D \frac{\partial N}{\partial z} d r
$$

Integrating the continuity equations (Equations (2.1) and (2.61)) with respect to $r$ and utilizing the fact that the radial transport of the process gas and the desired component must vanish at the rotor wall gives

$$
\begin{gathered}
r \hat{\rho} \hat{u}=\int_{r}^{a} \frac{\partial}{\partial z}(\hat{\rho} \hat{w}) r d r \\
r \hat{\rho} \hat{u} N+r J_{r}=\int_{r}^{a} \frac{\partial}{\partial z}\left(\hat{\rho} \hat{w} N+J_{z}\right) r d r
\end{gathered}
$$

Combining the previous two equations to eliminate the $r \hat{\rho} \hat{u}$ term gives

$$
r J_{r}=\int_{r}^{a} \frac{\partial}{\partial z}\left(\hat{\rho} \hat{w} N+J_{z}\right) r d r-N \int_{r}^{a} \frac{\partial}{\partial z}(\hat{\rho} \hat{w}) r d r
$$

Assuming that the term $\frac{\partial J_{z}}{\partial z}$ which contains the second partial derivative of $N$ with respect to $z$ is sufficiently small and substituting in for $J_{r}$ gives 


$$
\frac{\partial N}{\partial r}=-\frac{1}{r \hat{\rho} D} \int_{r}^{a} \frac{\partial}{\partial z}(\hat{\rho} \hat{w} N) r d r+\frac{N}{r \hat{\rho} D} \int_{r}^{a} \frac{\partial}{\partial z}(\hat{\rho} \hat{w}) r d r-\frac{\Delta M \Omega^{2} r}{R_{u} T_{0}} N(1-N)
$$

Substituting Equation (2.73) into Equation (2.69) gives

$$
\begin{array}{r}
\tau-\tau^{*} N(0, z)-\int_{r}^{a} \mathscr{G}\left[\frac{1}{r \hat{\rho} D} \int_{r}^{a}\left(\hat{\rho} \hat{w} \frac{\partial N}{\partial z}+N \frac{\partial(\hat{\rho} \hat{w})}{\partial z}\right) r d r+\frac{N}{r \hat{\rho} D} \int_{r}^{a} \frac{\partial(\hat{\rho} \hat{w})}{\partial z} r d r\right] d r \\
-\int_{0}^{a} \mathscr{G} \frac{\Delta M \Omega^{2} r}{R_{u} T_{0}} N(1-N) d r-\int_{0}^{a} 2 \pi r \hat{\rho} D \frac{\partial N}{\partial z} d r
\end{array}
$$

Because the radial concentration gradient is small compared to the axial concentration gradient, $N$ can be considered independent of $r$. Applying this assumption and letting $\bar{N}$ be the radially averaged concentration gives

$$
\left[\int_{0}^{a} \frac{\mathscr{G}^{2}}{2 \pi r \hat{\rho} D} d r+\int_{0}^{a} 2 \pi r \hat{\rho} D d r\right] \frac{d \bar{N}}{d z}=-\left[\int_{0}^{a} \mathscr{G} \frac{\Delta M \Omega^{2} r}{R_{u} T_{0}} d r\right] \bar{N}(1-\bar{N})-\left(\tau-\tau^{*} \bar{N}\right)
$$

Because the centrifuge is essentially isothermal the term $\hat{\rho} D$ can be considered a constant, giving

$$
\left[\frac{1}{2 \pi \hat{\rho} D} \int_{0}^{a} \frac{\mathscr{G}^{2}}{r} d r+\pi a^{2} \hat{\rho} D\right] \frac{d \bar{N}}{d z}=-\frac{\Delta M \Omega^{2}}{R_{u} T_{0}}\left[\int_{0}^{a} \mathscr{G} r d r\right] \bar{N}(1-\bar{N})-\left(\tau-\tau^{*} \bar{N}\right)
$$

For the case where $\tau^{*}<<L$, the circulation rate can be defined as

$$
L=\frac{1}{2} \int_{0}^{a} 2 \pi r|\hat{\rho} \hat{w}| d r
$$

Dividing Equation (2.76) by Equation (2.77) gives the final gradient equation 
Table 2.2: Separation terms in the gradient equation (Equation (2.78))

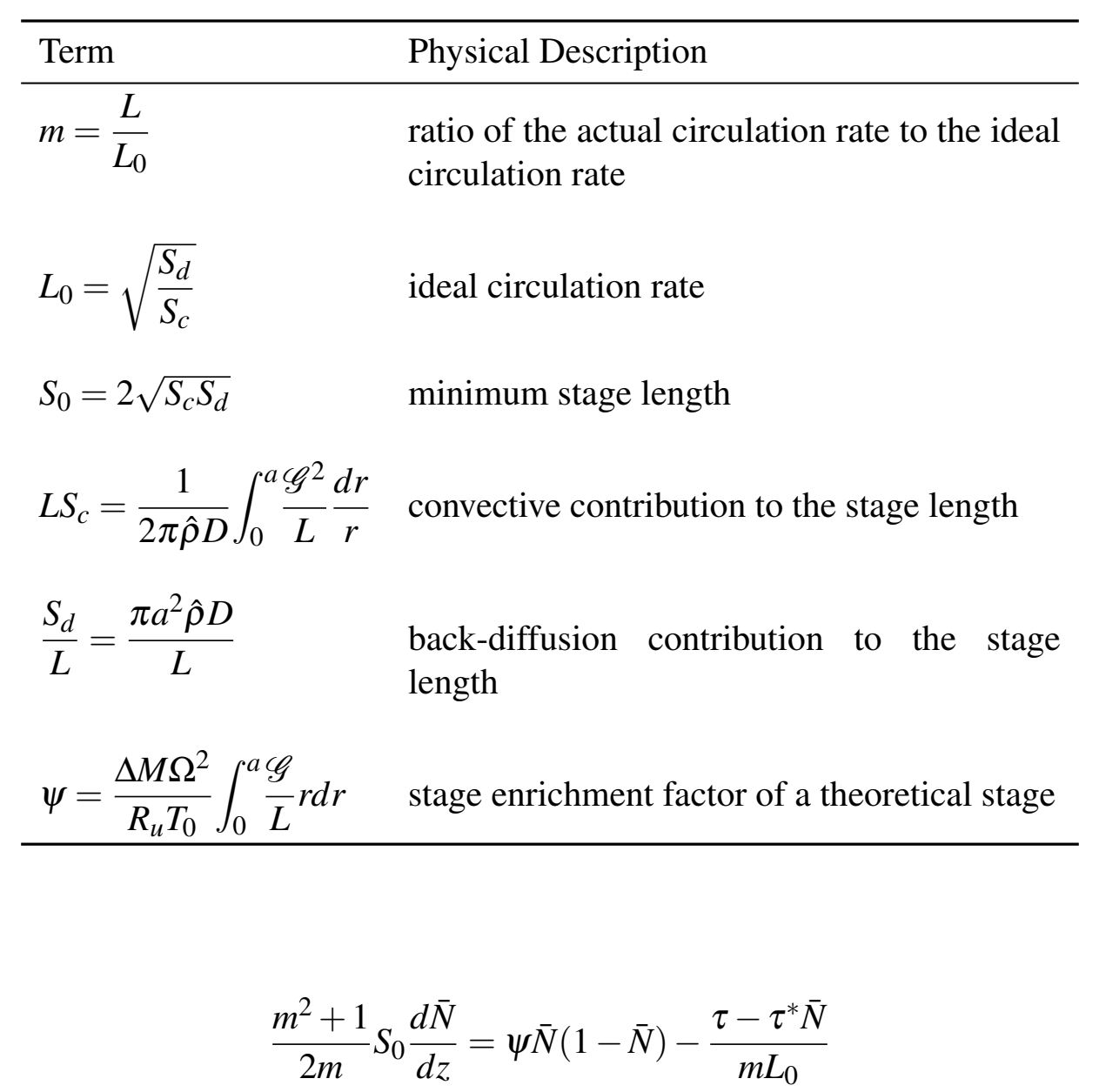

where the terms are defined in Table 2.2. In order to determine the concentration gradient over the entire centrifuge, Equation (2.78) must be solved for the enriching section and stripping section of the centrifuge.

The boundary conditions for Equation (2.78) are

$$
\begin{aligned}
\text { Enriching section: } & z=z_{F}, \quad \bar{N}=N_{c, 0} \\
z & =z_{P}, \quad \bar{N}=N_{c, P}
\end{aligned}
$$

Stripping section: $\quad z=0, \quad \bar{N}=N_{c, W}$

$$
z=z_{F}, \quad \bar{N}=N_{c, 0}
$$

where $N_{c, 0}$ is the concentration of the key isotope at the feed location of the centrifuge 
which may not be equal to the feed concentration.

\subsubsection{Separation Parameters}

The performance of a gas centrifuge can be classified by two parameters, the separation factor and the separative power. The overall separation factor is a measure of the enrichment gained across the centrifuge and is defined as

$$
\gamma=\frac{N_{c, P}}{1-N_{c, P}} \cdot \frac{1-N_{c, W}}{N_{c, W}}
$$

The separative power, $\delta U$, defined as the amount of useful work done by the centrifuge is

$$
\delta U=L_{c} V\left(N_{c, P}\right)+D_{c} V\left(N_{c, W}\right)-G_{c} V\left(N_{c, F}\right)
$$

where $G_{c}$ is the centrifuge feed rate, $N_{c, F}$ is the centrifuge feed concentration, and $V$ is the value function defined as

$$
V(N)=(2 N-1) \ln \left[\frac{N}{1-N}\right]
$$

The value function measures the value of a given concentration and the separative power represents the useful separative work per unit time performed by the centrifuge [38].

The theoretical maximum separative power that a gas centrifuge can achieve can be calculated by the formula derived by Paul Dirac [34]

$$
\delta U_{\max }=\frac{\pi \hat{\rho} D H}{2}\left[\frac{\Delta M(a \Omega)^{2}}{2 R_{u} T_{0}}\right]^{2}
$$

where $H$ is the separative length of the centrifuge and $\Delta M$ is the mass difference between the binary gas mixture [34]. In practice, the maximum separative power is never achieved, and the actual separative power can be determined by the separative efficiency [38] 


$$
E=\frac{\delta U_{\exp }}{\delta U_{\max }}
$$

The separative efficiency of a countercurrent gas centrifuge is the product of four factors: the flow pattern efficiency $\left(e_{F}\right)$, the circulation efficiency $\left(e_{C}\right)$, the ideality efficiency $\left(e_{I}\right)$, and the experimental efficiency $\left(e_{E}\right)$ [38].

The flow pattern efficiency depends on the shape of the axial velocity profile and is determined by

$$
e_{F}=\frac{4}{a^{4}} \frac{\left[\int_{0}^{a \mathscr{G}} \frac{\bar{L}}{L} d r\right]^{2}}{\int_{0}^{a} \frac{\mathscr{G}^{2}}{L^{2}} \frac{d r}{r}}
$$

The circulation efficiency represents the loss of separative capacity due to axial diffusion working against axial convection and is defined as

$$
e_{C}=\frac{m^{2}}{1+m^{2}}
$$

The ideality efficiency represents the difference between the shape of the square cascade representation of the centrifuge and an ideal cascade. It accounts for mixing of concentrations and suboptimal operation of the centrifuge. It can be shown that the maximum value for this efficiency is 0.81 [38]. The experimental efficiency includes phenomena not captured in the flow model, diffusion model, or other efficiencies.

Often in the literature, because gas centrifuge technology is mostly proprietary or classified, it is useful to develop theoretically designed centrifuge machines that researchers can compare models with. Two generic centrifuges have been developed at the Workshop on Gases in Strong Rotation and Workshop on Separation Phenomena in Liquids and Gases, the "Rome" centrifuge [83] and the "Iguaçu" centrifuge [84]. The Rome centrifuge represents a large, supercritical, high separative power machine that is derived from the United State design. The Iguaçu centrifuge is a smaller, subcritical machine which is based 
Table 2.3: Geometric and operating details of the Rome and Iguaçu centrifuges

\begin{tabular}{lcccc}
\hline Parameter & Variable & Unit & Rome & Iguaçu \\
\hline Radius & $a$ & $\mathrm{~cm}$ & 25 & 6 \\
Height & $H$ & $\mathrm{~cm}$ & 500 & 48 \\
Wall speed & $\Omega$ & $\mathrm{m} / \mathrm{s}$ & 600 & 600 \\
Average gas temperature & $T_{0}$ & $\mathrm{~K}$ & 320 & 300 \\
Wall pressure & $p_{w}$ & torr & 100 & 60 \\
Stratification parameter & $A^{2}$ & - & 23.82 & 25.40 \\
Maximum separative power & $\delta U_{\max }$ & $\mathrm{SWU} / \mathrm{yr}$ & 164.7 & 16.98 \\
Actual optimal separative power & $\delta U_{a c t, 0}$ & $\mathrm{SWU}_{\mathrm{yr}}$ & 53.54 & 4.41 \\
Efficiency & $E$ & $\%$ & 32.51 & 25.97 \\
Optimal feed rate & $G_{c, 0}$ & $\mathrm{mgUF}_{6} / \mathrm{s}$ & 281.21 & 28.81 \\
Optimal overall separation factor & $\gamma_{0}$ & - & 1.3069 & 1.2743 \\
\hline
\end{tabular}

on Russian design. The geometric and operating details of theses centrifuges are shown in Table 2.3.

For this dissertation, centrifuge performance is calculated using the Pancake code developed by Houston Wood. The Pancake code solves the nonhomogeneous Onsager equation (Equation (2.38)) using a separation of variables and eigenfunction expansion approach. The presence of perturbations such as feed material entering the centrifuge, product and tails material exiting the centrifuge, the scoop acting on the flow, and an applied temperature gradient, are modeled using sources and sinks. The Pancake code solves four linear flow perturbations

1. feed with all material removed through the top scoop

2. feed with all material removed through the bottom scoop

3. linear wall temperature gradient

4. scoop force

The code then combines these flows depending on the specified magnitude of the individual perturbations and solves the gradient equation (Equation (2.78)). An optimization routine in the code can combine the four flows in a proportion to maximize 
the centrifuge separative power. A driver for the Pancake code was developed by the author and William C. Witt for use in MATLAB. The new driver allows the user to completely run the Pancake code from MATLAB and specify a range of feed rates and cuts for developing performance maps.

Performance maps are typically used to show the separative performance of a gas centrifuge at different feed rates and cuts. A performance map can be developed in one dimension (performance versus feed rate) or two dimensions (performance versus feed rate and cut). The cut $(\theta)$ is the ratio of the centrifuge product rate to the centrifuge feed rate. These maps are used to characterize a particular centrifuge much like operative curves for pumps. The performance map is developed in Pancake using a two step procedure. First, the optimized operating point is found by specifying the centrifuge geometry and allowing the flow perturbations (feed rate, cut, wall temperature gradient, and scoop force) to vary until the separative power is maximized. Next, the wall temperature gradient and scoop force are fixed and the separative parameters are calculated over a range of centrifuge feed rate and cut.

Figure 2.4 shows the 1D performance maps for the Rome (Figure 2.4a) and the Iguaçu (Figure 2.4b) centrifuge as calculated using the Pancake code. As seen in Figure 2.4, the performance for both centrifuge follows the same trend. The separation factor peaks as the centrifuge feed rate goes to zero and decreases as the feed rate increases. The separative power approaches zero as the feed rate goes to zero, rises to a maximum value as the feed rate increases, and then begins to decrease with further increasing feed rate. The feed rate where the separative power is maximum is deemed the optimal point. The $2 \mathrm{D}$ performance maps for the Rome centrifuge are shown in Figure 2.5 and for the Iguaçu centrifuge in Figure 2.6. 


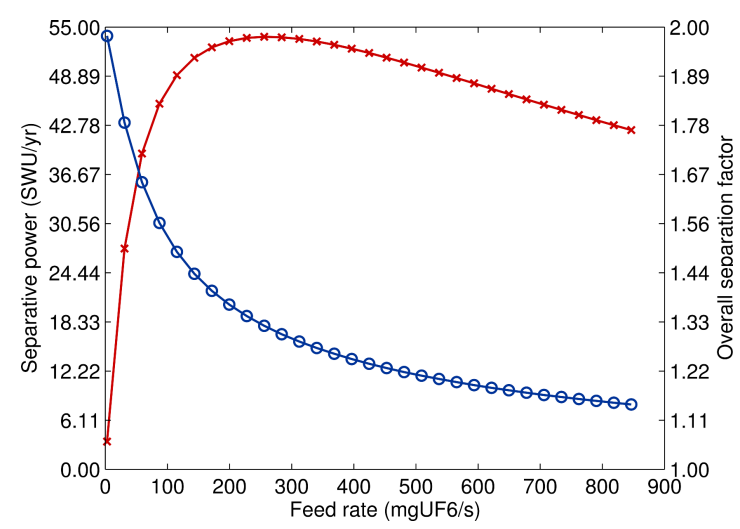

(a) Rome centrifuge

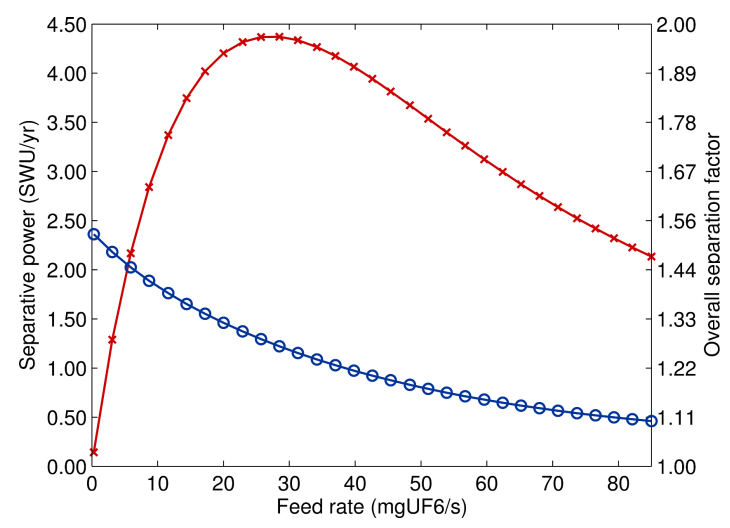

(b) Iguaçu centrifuge

Figure 2.4: 1D performance maps for the Rome and Iguaçu centrifuge. 1D performance maps detail the dependence of the separative power (red $\mathrm{x}$ ) and the separation factor (blue circle) versus the feed rate. For this case, the centrifuge cut is $\theta=0.5$. Typically, the separative power of the gas centrifuge approaches zero as the feed rate goes to zero, increases with increasing feed rate to a maximum point and then tails off. The feed rate where the separative power is maximized is deemed the optimal point. The separation factor reaches a maximum as the feed rate goes to zero and decreases with increasing feed rate.

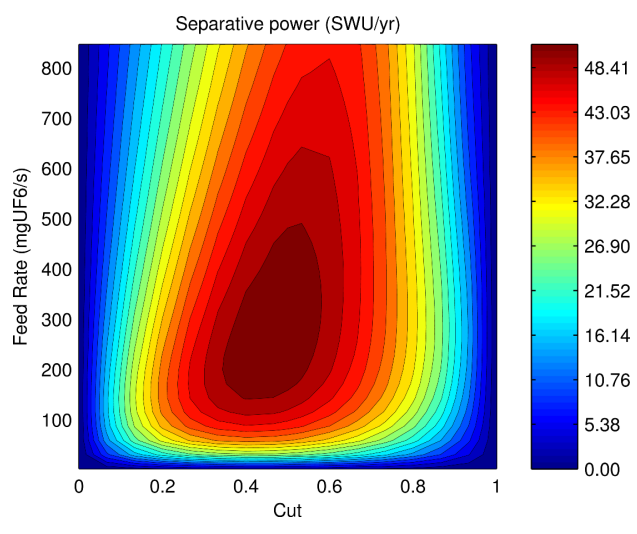

(a) $\delta U$ vs. $G_{c}, \theta$

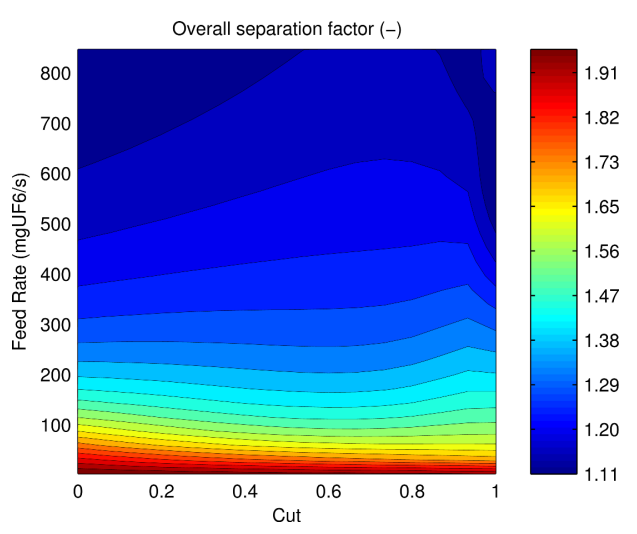

(b) $\gamma$ vs. $G_{c}, \theta$

Figure 2.5: 2D performance maps for the Rome centrifuge. 2D performance maps detail the separative performance of the centrifuge over a range of feed rates and cuts. The combination of feed rate and cut that maximize the centrifuge separative power is deemed the optimal operating point. 


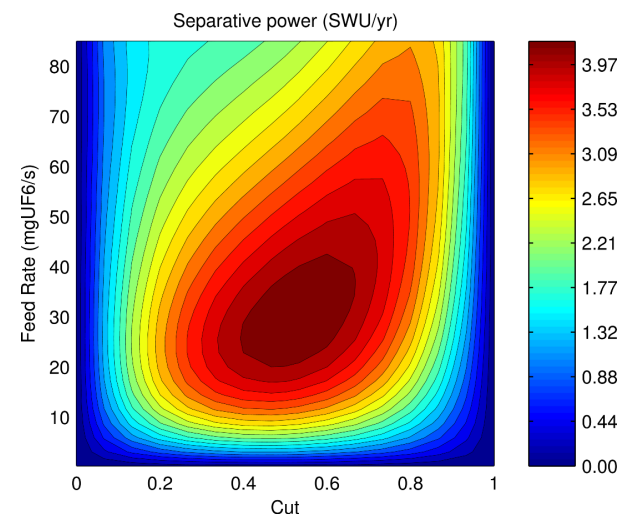

(a) $\delta U$ vs. $G_{c} \& \theta$

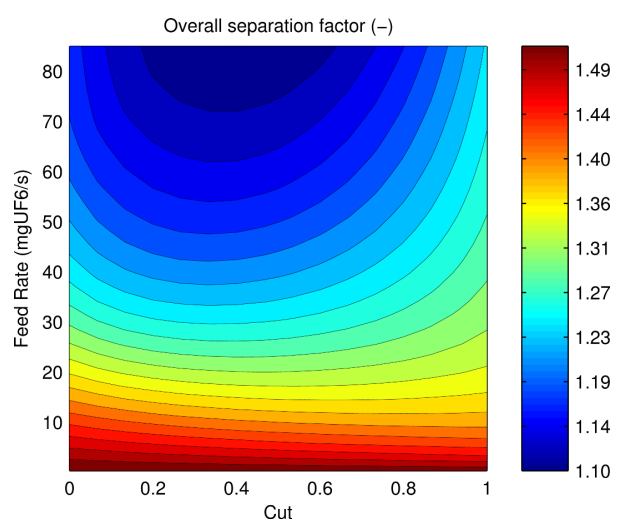

(b) $\gamma$ vs. $G_{c} \& \theta$

Figure 2.6: 2D performance maps for the Iguaçu centrifuge 


\section{Chapter 3}

\section{Cascade Theory}

\subsection{Overview}

Because the separative power of one centrifuge is relatively small in terms of mass producing fuel grade enrichments, centrifuges are often linked together in parallel and series to increase material throughput and enrichment level. Centrifuges linked in parallel are collectively referred to as a stage. As seen in Figure 3.1a, each stage is composed of several centrifuges and header piping for feed flow (blue), upflow (green), and downflow (red) which transport the collective flows of all the centrifuges to the next stage. Stages are connected in series to form the cascade. Stages can be connected in various ways, but the most practical configuration for a gas centrifuge cascade is the symmetric, countercurrent type (see Figure 3.1b). In this type of cascade, feed material enters the stage and leaves through an enriched upflow stream and a depleted downflow stream. The upflow stream is fed into the next stage and the downflow stream is fed into the previous stage. The cascade is divided into two sections, the enriching section and stripping section. The enriching section includes the feed stage and all stages above. The stripping section includes all stages below the feed stage.

The general theory of cascades has been developed in many classic references by Cohen [34], Hoglund et. al [38], Olander [39], Benedict et. al. [40], and Brigoli [85] and the theory presented in the chapter will closely follow these references. 


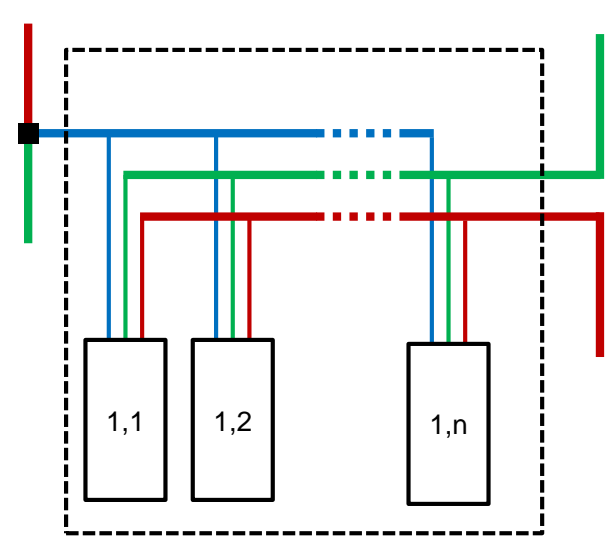

(a) Cascade stage flow diagram

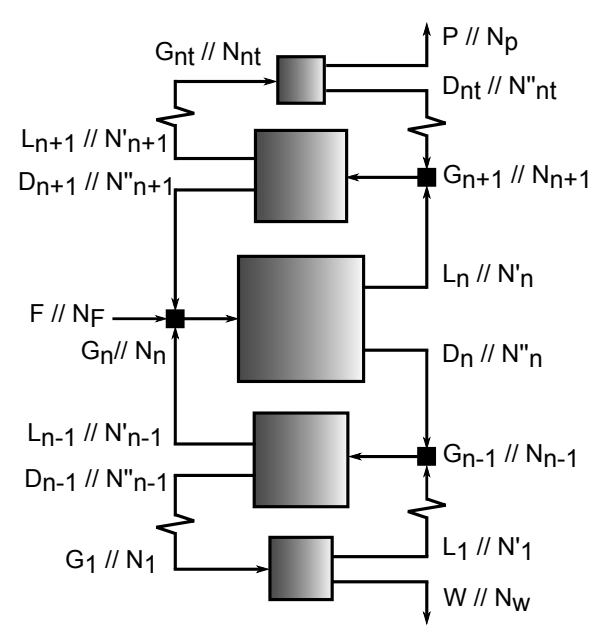

(b) Cascade stage diagram

Figure 3.1: Flow diagrams for a countercurrent cascade. Centrifuges are connected in parallel into stages where the individual centrifuge flows are collected into header piping. Stages are connected in series with the product stream of a stage feeding the next stage and the tails stream of a stage feeding the previous stage. The enriching section of the cascade includes the feed stage and all stages above. The stripping section includes all stages below the feed stage.

\subsection{General Cascades}

Consider a cascade with, $n=1 \cdots n_{E}$, stages in the enriching section and, $m=1 \cdots m_{E}$, in the stripping section. Assuming no losses, steady-state operation, and a binary mixture of isotopes, the overall and isotopic material balances across the entire cascade are

$$
\begin{gathered}
F=P+W \\
F N_{F}=P N_{P}+W N_{W}
\end{gathered}
$$

where $F$ is the cascade feed rate, $P$ is the cascade product rate, $W$ is the cascade tails rate, $N_{F}$ is the concentration of the desired isotope $\left({ }^{235} U\right.$ in the case of uranium enrichment) in the feed stream, $N_{P}$ is the concentration in the product stream, and $N_{W}$ is the concentration in the tails stream. Similarly for any stage in the cascade, 


$$
G_{n}=L_{n}+D_{n}
$$

$$
G_{n} N_{n}=L_{n} N_{n}^{\prime}+D_{n} N_{n}^{\prime \prime}
$$

where $G_{n}$ is the stage feed rate, $L_{n}$ is the stage upflow rate, $D_{n}$ is the stage downflow rate, $N_{n}$ is the stage feed concentration, $N_{n}^{\prime}$ is the stage upflow concentration, $N_{n}^{\prime \prime}$ is the stage downflow concentration, and the subscript $n$ represents the stage number. These equations are appropriate for stages in both the enriching section and stripping section of the cascade. The flow rates defined here are the collective flow rates of all of the centrifuges in the stage.

The stage cut can be defined as the ratio of upflow rate to feed rate

$$
\theta_{n}=\frac{L_{n}}{G_{n}}=1-\frac{D_{n}}{G_{n}}
$$

Substituting Equation (3.5) into Equation (3.4) yields a relationship between the cut and the stage concentrations

$$
\theta_{n}=\frac{N_{n}-N_{n}^{\prime \prime}}{N_{n}^{\prime}-N_{n}^{\prime \prime}}
$$

Three separation factors can be defined for the stage, the overall $(\gamma)$, heads $(\alpha)$, and tails $(\beta)$

$$
\begin{aligned}
& \gamma_{n}=\frac{R\left(N_{n}^{\prime}\right)}{R\left(N_{n}^{\prime \prime}\right)}=\frac{N_{n}^{\prime}}{1-N_{n}^{\prime}} \cdot \frac{1-N_{n}^{\prime \prime}}{N_{n}^{\prime \prime}} \\
& \alpha_{n}=\frac{R\left(N_{n}^{\prime}\right)}{R\left(N_{n}\right)}=\frac{N_{n}^{\prime}}{1-N_{n}^{\prime}} \cdot \frac{1-N_{n}}{N_{n}} \\
& \beta_{n}=\frac{R\left(N_{n}\right)}{R\left(N_{n}^{\prime \prime}\right)}=\frac{N_{n}}{1-N_{n}} \cdot \frac{1-N_{n}^{\prime \prime}}{N_{n}^{\prime \prime}}
\end{aligned}
$$


where $R(N)$ is the abundance ratio. From Equations (3.7) - (3.9) it follows that $\gamma_{n}=\alpha_{n} \cdot \beta_{n}$.

The stage concentrations can be calculated by way of the gradient equation, which is obtained by combining the equilibrium-line equations and the operating-line equations (cascade material balances) [86]. The equilibrium-line equations are determined by rearranging the definition of the overall separation factor to find

$$
N_{n}^{\prime}-N_{n}^{\prime \prime}=\frac{\psi_{n} N_{n}^{\prime \prime}\left(1-N_{n}^{\prime \prime}\right)}{1+\psi_{n} N_{n}^{\prime \prime}}
$$

where, $\psi_{n}=\gamma_{n}-1$, is the stage enrichment factor. In the enriching section of the cascade, the operating-line equations are found by taking a material balance around the top of the cascade and just about the $n^{\text {th }}$ stage. The overall material balance is

$$
L_{E, n}=D_{E, n+1}+P
$$

and the isotopic material balance is

$$
L_{E, n} N_{E, n}^{\prime}=D_{E, n+1} N_{E, n+1}^{\prime \prime}+P N_{P}
$$

The previous two equations can be combined to give the operating-line equation in the enriching section of the cascade

$$
L_{E, n} N_{E, n}^{\prime}=\left(L_{n}-P\right) N_{E, n+1}^{\prime \prime}+P N_{P}
$$

For the stripping section of the cascade, a similar approach yields the stripping section operating-line equations

$$
\left(W+L_{S, m-1}\right) N_{S, m}^{\prime \prime}=W N_{W}+L_{S, m-1} N_{S, m-1}^{\prime}
$$

The operating-line equations and equilibrium-line equations can be combined to give the exact finite difference form of the gradient equations for the enriching section of the 
cascade

$$
N_{E, n+1}^{\prime \prime}-N_{E, n}^{\prime \prime}=\frac{L_{E, n}}{L_{E, n}-P}\left[\frac{\psi_{E, n} N_{E, n}^{\prime \prime}\left(1-N_{E, n}^{\prime \prime}\right)}{1+\psi_{E, n} N_{E, n}^{\prime \prime}}-\frac{P}{L_{E, n}}\left(N_{P}-N_{E, n}^{\prime \prime}\right)\right]
$$

and the stripping section of the cascade

$$
N_{S, m+1}^{\prime \prime}-N_{S, m}^{\prime \prime}=\frac{L_{S, m}}{L_{S, m}+W}\left[\frac{\psi_{S, m} N_{S, m}^{\prime \prime}\left(1-N_{S, m}^{\prime \prime}\right)}{1+\psi_{S, m} N_{S, m}^{\prime \prime}}-\frac{W}{L_{S, m}}\left(N_{S, m}^{\prime \prime}-N_{W}\right)\right]
$$

Equations (3.1), (3.2), (3.15) and (3.16) combined with knowledge of the number of stages, stage upflow rates (or cuts), cascade feed rate, cascade feed concentration, and the centrifuge separation factor (as a function of feed rate and cut) are sufficient to determine the concentration gradient in the cascade. This type of calculation will be referred to as a productivity analysis in subsequent chapters in this dissertation.

In addition to the stage concentrations, the separative power of the cascade and each stage are important quantities. For the entire cascade, the separative power can be found as

$$
\Delta U=P \cdot V\left(N_{P}\right)+W \cdot V\left(N_{W}\right)-F \cdot V\left(N_{F}\right)
$$

where $V(N)$ is the value function defined as

$$
V(N)=(2 N-1) \ln \left[\frac{N}{1-N}\right]
$$

Similarly, the separative power for a general stage is

$$
\delta U_{n}=L_{n} \cdot V\left(N_{n}^{\prime}\right)+D_{n} \cdot V\left(N_{n}^{\prime \prime}\right)-G_{n} \cdot V\left(N_{n}\right)
$$




\subsubsection{Approximations to the Gradient Equations}

There are several conditions for which simplifying approximations can be made to the gradient equations. The first condition, when the enrichment along the cascade is small and gradual, the concentration and stage number can be considered continuous, and the term $N_{n+1}-N_{n}$ can be replaced by $\frac{d N}{d n}[85,86]$. This assumption gives the differential form of the gradient equations

$$
\begin{aligned}
& \frac{d N_{E}^{\prime \prime}}{d n}=\frac{L_{E}}{L_{E}-P}\left[\frac{\psi N_{E}^{\prime \prime}\left(1-N_{E}^{\prime \prime}\right)}{1+\psi N_{E}^{\prime \prime}}-\frac{P}{L_{E}}\left(N_{P}-N_{E}^{\prime \prime}\right)\right] \\
& \frac{d N_{S}^{\prime \prime}}{d m}=\frac{L_{S}}{L_{S}+W}\left[\frac{\psi N_{S}^{\prime \prime}\left(1-N_{S}^{\prime \prime}\right)}{1+\psi N_{S}^{\prime \prime}}-\frac{W}{L_{S}}\left(N_{S}^{\prime \prime}-N_{W}\right)\right]
\end{aligned}
$$

Next, in the case where the stage separation factor is nearly one, both the cascade product rate and tails rate must also be much smaller than the stage upflow rate everywhere in the cascade except near the withdrawal points [86]. This is justified by considering the minimum upflow rate, which will be discussed later. The assumption of a small separation factor also gives rise to an approximate form of the equilibrium-line equation. Expanding the denominator in Equation (3.10) in a power series, one finds

$$
N^{\prime}-N^{\prime \prime} \approx(\gamma-1) N^{\prime \prime}\left(1-N^{\prime \prime}\right)
$$

thus giving approximate forms of the differential gradient equations

$$
\begin{aligned}
& \frac{d N_{E}^{\prime \prime}}{d n}=\psi_{E} N_{E}^{\prime \prime}\left(1-N_{E}^{\prime \prime}\right)-\frac{P}{L_{E}}\left(N_{P}-N_{E}^{\prime \prime}\right) \\
& \frac{d N_{S}^{\prime \prime}}{d m}=\psi_{S} N_{S}^{\prime \prime}\left(1-N_{S}^{\prime \prime}\right)-\frac{W}{L_{S}}\left(N_{S}^{\prime \prime}-N_{W}\right)
\end{aligned}
$$

where $\gamma, N^{\prime \prime}$, and $L$ are functions of $n$ and $m$. 


\subsubsection{Limiting Conditions}

For a given cascade product concentration and slope of the operating-line, there exists a minimum value of achievable concentration at the intersection of the operating-line and equilibrium-line [86]. This intersection is called the pinch-point, and to reach a specified value of $N$ in the cascade, there is a minimum value of the slope of the operating-line required [86]. This can also be expressed as a minimum upflow rate for a given cascade product rate. At the pinch-point, the enrichment per stage will go to zero, and thus the minimum upflow rate can be found by setting, $\frac{d N}{d n}$, or, $N_{n+1}^{\prime \prime}-N_{n}^{\prime \prime}$, to zero. Considering Equations (3.15) and (3.16), the minimum upflow rates in the enriching section and stripping section of the cascade are

$$
\begin{gathered}
L_{E, n, \min }=\frac{P\left(N_{P}-N_{E, n}^{\prime \prime}\right)\left(1+\psi_{E, n}\right)}{\psi_{E, n} N_{E, n}^{\prime \prime}\left(1-N_{E, n}^{\prime \prime}\right)} \\
L_{S, n, \min }=\frac{W\left(N_{S, m}^{\prime \prime}-N_{W}\right)\left(1+\psi_{S, m}\right)}{\psi_{S, m} N_{S, m}^{\prime \prime}\left(1-N_{S, m}^{\prime \prime}\right)}
\end{gathered}
$$

Examining the previous two equations, the upflow rates will be much larger than the cascade product rate and tails rate everywhere in the cascade except near the withdrawal points.

\subsection{The Ideal Cascade}

An ideal cascade is defined as the most efficient possible cascade (not necessarily the most cost effective). In order to achieve this, there needs to be no losses in separative power due to mixing, and every centrifuge must be operating at the same optimal design point. In terms of stage variables, this can be expressed as 


$$
\begin{gathered}
N_{n}=N_{n-1}^{\prime}=N_{n+1}^{\prime \prime} \\
\alpha_{n}=\beta_{n}=\sqrt{\gamma_{n}}=\alpha_{0}=\beta_{0}=\sqrt{\gamma_{0}}
\end{gathered}
$$

Applying the assumptions in Equations (3.27) and (3.28), the feed concentration gradient in the enriching section of the cascade can be found to be

$$
N_{E, n}=\frac{R\left(N_{F}\right) \alpha_{0}^{n-1}}{1+R\left(N_{F}\right) \alpha_{0}^{n-1}}
$$

Similarly, for the stripping section

$$
N_{S, n}=\frac{R\left(N_{F}\right) \alpha_{0}^{n-n_{s}-1}}{1+R\left(N_{F}\right) \alpha_{0}^{n-n_{s}-1}}
$$

These equations are useful if the number of stages in the cascade are known. Suppose there are target enrichments for the product and tails streams of the cascade, Equations (3.29) and (3.30) can be rearranged to find an expression for the number of stages needed

$$
\begin{gathered}
n_{E}=\ln \left[\frac{R\left(N_{P}\right)}{R\left(N_{F}\right)}\right] \cdot \frac{1}{\ln \left[\alpha_{0}\right]}+1 \\
n_{S}=\ln \left[\frac{R\left(N_{F}\right)}{R\left(N_{W}\right)}\right] \cdot \frac{1}{\ln \left[\alpha_{0}\right]}
\end{gathered}
$$

A relationship between the cut and the separation factor can be found as

$$
\theta_{n}=\frac{1+\left(\alpha_{0}-1\right) N_{n}}{\alpha_{0}+1}
$$

which is valid for both the enriching and stripping sections of the cascade. In the enriching section, taking a material balance around the top of the cascade and a general stage, $n$, gives 


$$
L_{E, n} N_{E, n}^{\prime}=\left(L_{E, n}-P\right) N_{E, n}+P N_{P}
$$

Combining with Equations (3.5) and (3.33) gives an expression for the stage feed rates in the enriching section of the cascade

$$
G_{E, n}=P \cdot \frac{\alpha_{0}+1}{\alpha_{0}-1} \cdot \frac{N_{P}-N_{E, n}}{N_{E, n}\left(1-N_{E, n}\right)}
$$

A similar expression can be derived for the stripping section of the cascade

$$
G_{S, n}=W \cdot \frac{\alpha_{0}+1}{\alpha_{0}-1} \cdot \frac{N_{S, n}-N_{W}}{N_{S, n}\left(1-N_{S, n}\right)}
$$

The number of centrifuges in each stage can then be found as

$$
M_{n}=G_{n} / G_{0}
$$

The ideal cascade can now be determined through a combination of specified parameters and the above equations. For instance, one combination is: the cascade feed rate, feed concentration, optimal centrifuge separation factor, optimal centrifuge feed rate, and number of stages combined with Equations (3.28), (3.31), (3.32), (3.33), (3.35), (3.36), and (3.37). 


\section{Chapter 4}

\section{GCEPs and Proliferation}

\subsection{Components of a GCEP}

In Chapter 1, a brief introduction into the enrichment process at a gas centrifuge plant was given. In this chapter, the components and operation of a gas centrifuge plant is further discussed and proliferation scenarios are defined. The fine details of a gas centrifuge enrichment plant vary from facility to facility, but there are major components that are necessary at every plant. From a modeling perspective, the most important sections of the enrichment plant are the cascade hall and feed and withdrawal point, for which a generic flow diagram is shown in Figure 4.1. The flow of material begins by physically transporting feed cylinders that are full of solid $\mathrm{NUF}_{6}$ from the cylinder storage area to the feed area. The cylinders are placed in an autoclave which heats the cylinder to convert the solid $\mathrm{UF}_{6}$ to a gas. The gas is then pumped through an optional sampling station and impurity removal station where an operator can check the quality of the feed entering the cascade hall and remove impurities such as light gases. The gas then enters a holdup drum to ensure continuous flow to the cascades when there are cylinder changes and flows into the cascade hall at a controlled rate. Feed material travels through a main header pipe and is dispersed to the cascades. A grouping of cascades is called a production unit. Larger enrichment plants may have multiple production units with multiple cascades, providing flexibility to plant operations and allowing the operator to meet various customer demands [8]. The flow to and from each cascade can be controlled by valving. Flexible cascades allow the 


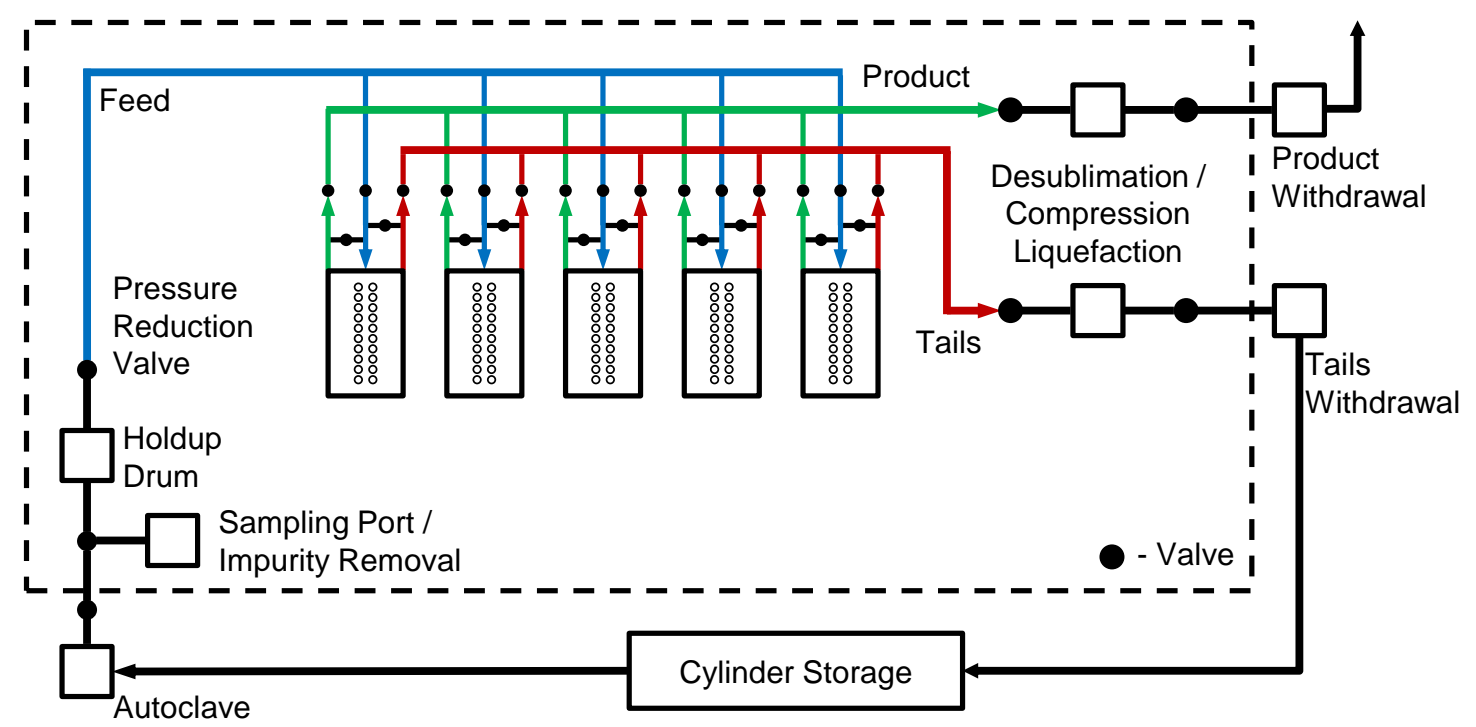

Figure 4.1: Flow diagram of a generic gas centrifuge enrichment plant. Feed cylinders are transported from the cylinder storage area to an autoclave where solid $\mathrm{UF}_{6}$ is heated and converted to a gas. The gaseous $\mathrm{UF}_{6}$ is pumped though a sampling port and impurity removal station and into a holdup drum. The pressure of the gas is reduced and then fed into the cascades. Cascades are typically joined together in production units. Depending on the size of the plant, there can be one or several production units. The product flows of the cascades are transported through a product header and collected in product cylinders. Similarly, the tails flows are collected and stored on-site. ${ }^{1}$

operator to recycle product and tails flow back into the feed stream. Once the material travels through the cascade, enriched material is pumped into the production unit product header and depleted material into the tails header. The flow is then collected by either desublimation or compression/liquefaction and is ultimately transferred to cylinders [8].

Figure 4.2 shows the flow diagram inside an example symmetric, countercurrent cascade with 8 stages. In a real cascade, centrifuges are often laid out in two parallel rows with header piping connecting the centrifuge into stages [8]. Feed flow from the production header pipe enters the cascade at the feed point (blue square) of the feed stage and is dispersed into each centrifuge in that stage. Figure 4.3 shows the flow in a particular stage of the cascade. The feed stream enters the centrifuge and enriched product is withdrawn at the top and depleted tails at the bottom. The product stream of each centrifuge is

\footnotetext{
${ }^{1}$ This figure has been derived from H. A. Elayat et. al., Gas Centrifuge Enrichment Plant Safeguards System Modeling [87] and Nuclear Material Safeguards for Uranium Enrichment Plants [8].
} 


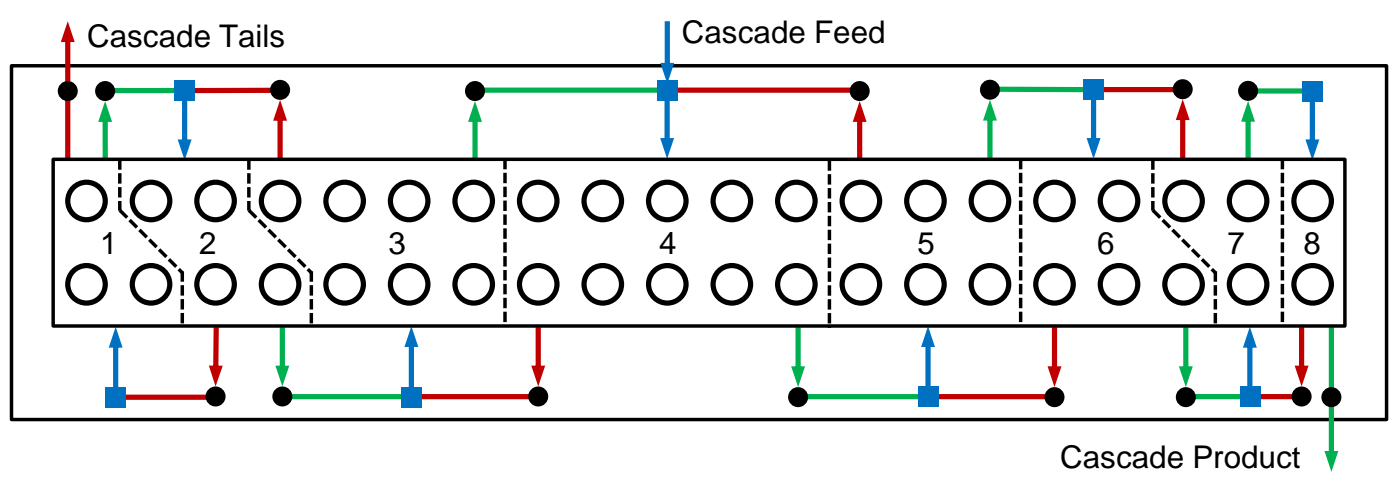

Figure 4.2: Flow diagram of a real, one-up, one-down symmetric cascade. Typically, centrifuges are arranged in rows with the stages created by piping. In this example, an 8 stage cascade is formed with 40 centrifuges. ${ }^{2}$

collected in the stage product header and flows to the next stage feed point. Along this flow path, there may be service access connections that allow the operator to withdraw (or add) material using a portable system. Also along this flow path is some means of actively or passively controlling the flow rate (such as a valve). Both these features are represented by a black circle on the diagram. Similarly, the tails stream is collected and flows to the previous stage feed point. In the event of centrifuge failure, the piping to the centrifuge may be crimped, isolating the malfunctioning centrifuge from the rest of the stage.

\subsection{Proliferation and Misuse of a GCEP}

There are many possible proliferation scenarios that can be considered in a GCEP, but most can be categorized into two general cases, the production of material with higher than declared enrichment, and the diversion or overproduction of declared material. Some of these proliferation scenarios are shown in Figure 4.4.

A cascade and cascade hall can be manipulated in many ways to produce material with higher than declared enrichments. These operations can either be achieved by modifying existing piping, adding new piping, or by adjusting flow controls.

\footnotetext{
${ }^{2}$ This figure has been derived from Nuclear Material Safeguards for Uranium Enrichment Plants [8].
} 


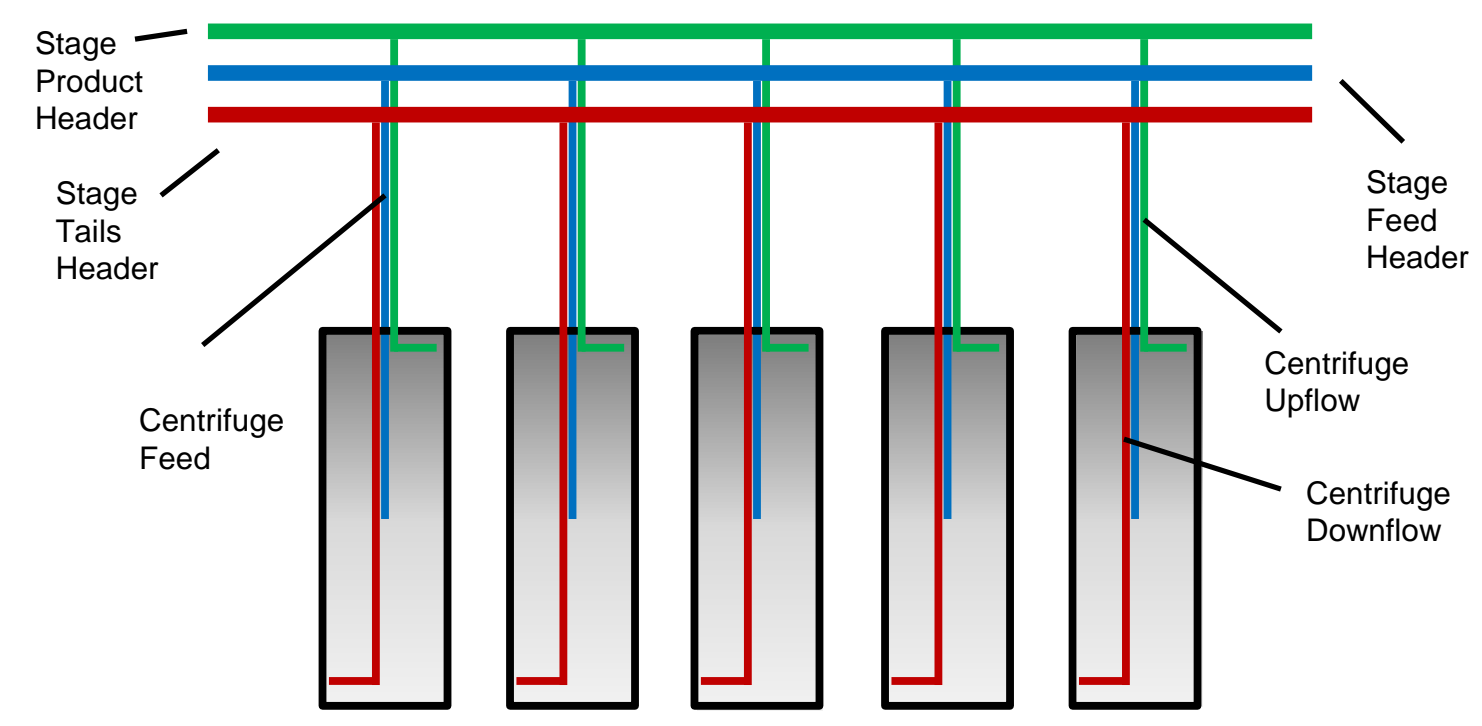

Figure 4.3: Flow diagram of a generic stage in a cascade. Centrifuges are linked to header piping by small pipes. The feed material enters the stage and is distributed to each centrifuge. The product and tails header pipes collect the individual product and tails streams from each centrifuge.

Cascade reconfiguration, depicted in Figure 4.4a, is a method by which the piping in the cascade is physically modified in a such a way to lengthen the cascade. The greater the number of stages in the cascade, the greater the enrichment. This can be achieved by reducing the number of centrifuges in some stages and adding them to the top of the cascade or by adding centrifuges to the cascade.

Partial or total recycling of the cascade product, shown in Figure 4.4b, can be achieved by closing off the valve from the cascade product header to the production unit product header and opening the valve between the cascade product header and cascade feed header. This allows more enriched material to be introduced at the feed stage, increasing the concentration of ${ }^{235} \mathrm{U}$ at the feed point. This method is inefficient due to the large mismatch between the cascade product enrichment and feed enrichment.

In the above situations, ES is considered the best deterrent and best means for detecting the over enrichment of material. However, in the event that enrichments greater than declared are found at a plant, it is difficult to know for sure if the operator is intentionally 


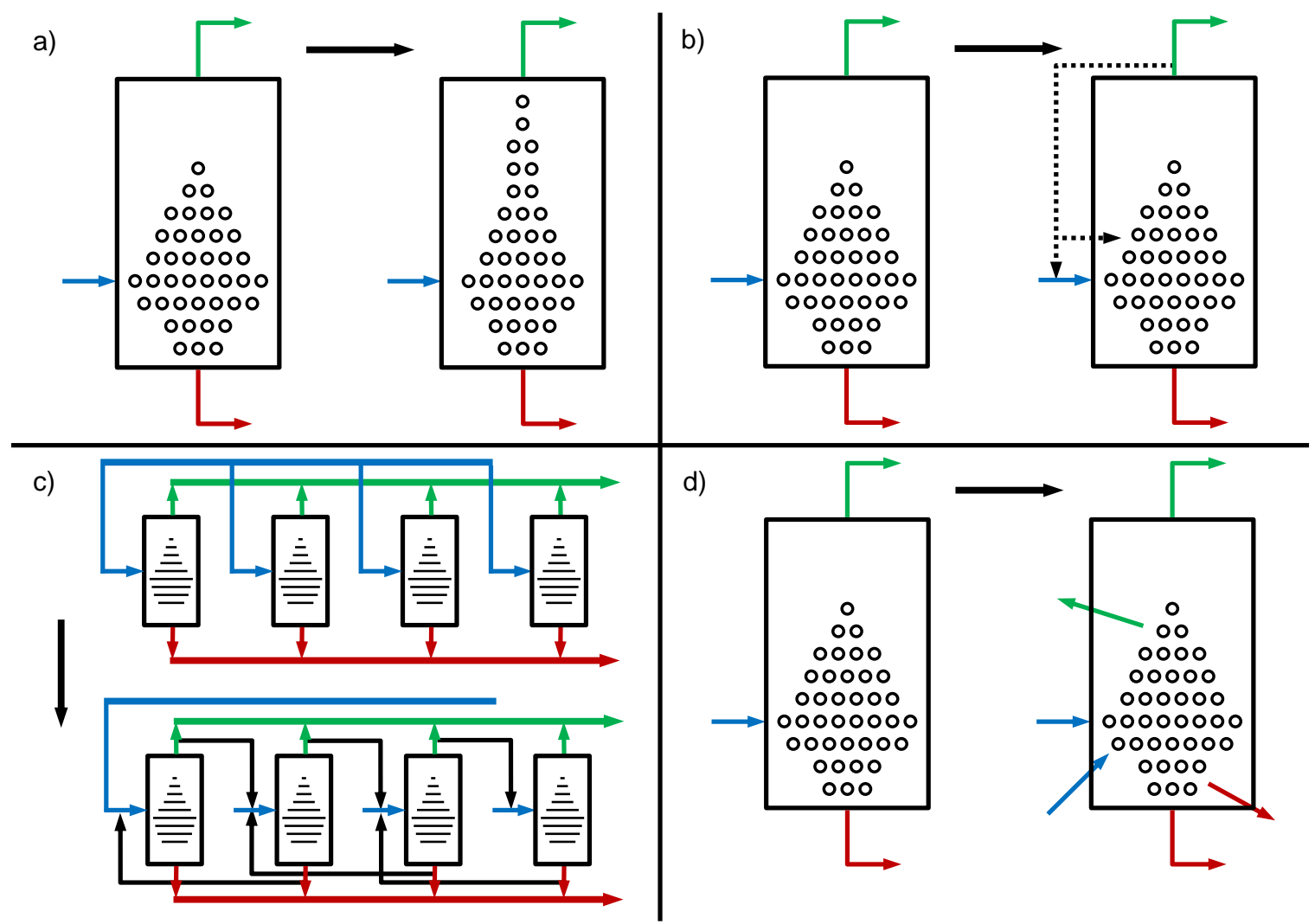

Figure 4.4: Generic proliferation scenarios that may occur in a GCEP. a) One way to increase the product enrichment out of the cascade is to lengthen the cascade by reconfiguring the cascade. Centrifuges can be taken from the middle stages of the cascade and used to add stages to the top of the cascade. b) Another way to increase the cascade product enrichment is to partially or fully recycle the product stream into either the feed stage or some other stage of the cascade. c) The most efficient way to produce a higher enrichments in a cascade hall is to connect cascades in parallel and series. d) Overproduction or diversion of material can be achieved by introducing side-streams through sampling ports in a stage.

over-enriching or if the discrepancy is due to quasi-transient phenomena such as cascade startup.

Cascade interconnection, shown in Figure 4.4c, involves configuring several cascades in a production unit to operate in a cascade-like manner. Cascades can be grouped together in parallel to form different steps of the enrichment process similar to centrifuges in a stage. This procedure begins by selecting a group of cascades to operate in the first step. For this grouping, keep the valve between the production unit feed header pipe open and close off the valve for the other cascades. Next, feed the collective product from the first 
step cascades into the next grouping of cascades (step 2). The product of this grouping can be used to feed the next grouping and so on. This scenario can also involve feeding the tails from a group back into the previous grouping, although matching enrichments is difficult. Since this scenario involves intensive reconfiguration, it would most likely be feasible only for producing weapons-grade uranium (WGU) in a breakout scenario.

Another scenario that doesn't involve modifying the cascade is flow reduction. In this case, the operator may adjust the valving in the cascade to reduce the upflow rates in the cascade. This has the effect of reducing the centrifuge feed rates in the enriching section of the cascade, thus increasing the product enrichment. This operation can be performed with and without modifying the centrifuge to enhance separative performance at lower flow rates.

The second general proliferation scenario, the over production or diversion of material at declared enrichments can be achieved by falsifying material flows or by introducing undeclared feed material into a cascade, shown in Figure 4.4d. Undeclared feed can be introduced in the sampling port between the autoclave and holdup drum or in the sampling ports in the cascade. In an effort to remain undetected, the operator can also withdraw material from the top and bottom of the cascade in the correct proportion to keep the cascade material balance satisfied. The diverted product material can then be used to feed a clandestine enrichment plant to further achieve WGU.

\subsection{Relevant Work}

Due to the sensitive nature of enrichment research and intelligence, there are very few studies in the literature that deal with modeling and simulation of gas centrifuge cascades.

Glaser [32] developed a method to simulate important breakout scenarios including batch recycling and cascade interconnection. Glaser used the Rätz analytical model to characterize the performance of the centrifuge and assumes a constant cut of 0.46 in his 
cascade model. Glaser shows that cascade interconnection is much more efficient than batch recycling for cases where natural feed is only available.

Delbeke [88] developed a method to analyze the separative power loss of a cascade when external cascade parameters are changed. He shows how this loss can be minimized by redistributing centrifuges in the cascade allowing a more realistic assessment of proliferation potential. Delbeke [89] used this method to assess the validity of open source data related to the Natanz Fuel Enrichment Plant in Iran.

Elayat et. al. [87] developed a flow model of a generic enrichment facility using the commercial software Extend. They study the scenario of skimming product material from one of the cascades in a production unit. Isotope separation in the plant is not considered.

Delbeke et. al. $[90,91]$ developed a real-time mass balance model (RTMES) to analyze load cell information continuously collected from feed and withdrawal stations. They show signatures of misuse for several diversion scenarios in which the cascade external flows are altered. Cleary and Carchon [92] extend the RTMES model of Delbeke et. al. to include plant reconfiguration and batch recycling. These studies do not model the isotope separation in the cascade making them unable to determine enrichment signatures of offnormal operation.

Rousseau et. al. [93] developed a fluid dynamics and isotope separation model of a gas centrifuge cascade to simulate the transient operation of a gas centrifuge plant. The fluid model was constructed using the commercial code Flownex, a thermal-fluid network simulation tool. A simple, lumped parameter model was used to incorporate the centrifuge base state flow physics. The homogeneous Onsager equation with linear wall drive and the gradient equation were used to characterize the separation performance of the centrifuge. No detail is provided about the cascade isotope separation model. This simulation tool was developed to gain an understanding of cascade design and to study normal operating conditions in a centrifuge plant, no safeguards or nonproliferation issues were considered. The flow model developed by Rousseau et. al. is more refined than the one that will 
be presented in this dissertation, but in good agreement is found between the two in a verification case in Chapter 8. The work of Rousseau et. al. was performed concurrent and independent of this work. 


\section{Chapter 5}

\section{Quantifying Reasonable Enrichment Levels in a Cascade}

\subsection{Overview}

As discussed in Chapter 1, one of the safeguards technologies that IAEA inspectors employ during inspections at gas centrifuge enrichment plants is environmental sampling. Environmental sampling is used to provide assurance that undeclared enrichment activities, such as over-enrichment, are not taking place. As shown in Chapter 2, the separative performance of a gas centrifuge is heavily dependent on the centrifuge feed rate.

During startup of a cascade, as material is initially fed into the cascade, the stage flow rates and centrifuge feed rate will increase incrementally until the designed flow rates are reached. When the centrifuge feed rate is below the designed operating point, the centrifuge separation factor will be greater than during normal operation and in turn, the concentrations found in the cascade will be greater than expected. If material leaks out of the cascade during startup, this transient enrichment spike can make interpreting environmental sampling results difficult. For instance, if during a swipe test material is found with enrichments higher than declared, it is difficult to know if the discrepancy is reasonable or if the operator is attempting to over-enrich material.

Therefore, it is imperative to quantify what a reasonable discrepancy between measured and expected values should be. In this chapter, a method for predicting the full range 
of concentrations that may be present in a particular cascade due to startup is detailed. To demonstrate what a discrepancy might look like, two types of centrifuge models are considered, one case representing normal operation and one which might represent intentional misuse of the centrifuge cascade. In the event that an operator is over-enriching intentionally, this method can also be used to predict how much material can be obtained at a particular concentration, an important quantity for use in nonproliferation studies. A large portion of this chapter appears in "A Study of Multicomponent Streams in Off-Design Centrifuge Cascades" in Separation Science and Technology [94].

\subsection{Cascade Design and Off-Normal Operation}

In order to predict the full range of concentrations that may be present in a particular cascade, details of the cascade structure and flow rates are required. This may be known in practice, but for this study, one must be developed. To create a cascade, first an ideal cascade calculation is performed by specifying the cascade product rate, target product concentration, and target tails concentration (see Section 3.3 for the details of this calculation). It is assumed that the cascade feed is natural uranium and the performance of the centrifuge is known by way of a performance map. To create a more realistic cascade, the cascade is squared-off by rounding the number of centrifuges per stage to the nearest integer. This slightly changes the centrifuge feed rate, altering the performance of the machine. The stage concentrations for the squared-off cascade can be calculated by performing a productivity analysis.

In Chapter 3, cascade theory was developed under the assumption that the composition of the material in the cascade was binary. In practice, this is generally not the case and there are several minor isotopes of uranium that can play an important role in the analysis of environmental sampling results. There are two types of feed stock available for enriching uranium, commercial natural or reprocessed (i.e. uranium that is recovered from nuclear 
Table 5.1: Typical uranium isotope concentrations in commercial natural uranium

\begin{tabular}{cccc}
\hline Isotope & i & $\begin{array}{c}\text { Commercial Natural Uranium } \\
\text { (at. frac.) }\end{array}$ & $\begin{array}{c}\text { Reprocessed Uranium } \\
\text { (at. frac.) }\end{array}$ \\
\hline${ }^{232} \mathrm{U}$ & 1 & $1.000 \times 10^{-11}$ & $5.000 \times 10^{-10}$ \\
${ }^{234} \mathrm{U}$ & 2 & $6.200 \times 10^{-5}$ & $2.200 \times 10^{-4}$ \\
${ }^{235} \mathrm{U}$ & 3 & $7.200 \times 10^{-3}$ & $1.220 \times 10^{-2}$ \\
${ }^{236} \mathrm{U}$ & 4 & $2.000 \times 10^{-5}$ & $4.200 \times 10^{-4}$ \\
${ }^{238} \mathrm{U}$ & 5 & $9.927 \times 10^{-1}$ & $9.872 \times 10^{-1}$ \\
\hline
\end{tabular}

waste). The difference between the isotopic composition of these two feed types is shown in Table 5.1.

Therefore, the productivity analysis will be extended to include the multicomponent case [95-97]. Consider a material containing $J=5$ isotopes as detailed in Table 5.1, a number of overall separation factors can be defined as

$$
\gamma_{i}=\gamma_{u}^{238-M_{i}}
$$

where $M_{i}$ is the mass number of isotope $i$ and $\gamma_{u}$ is the unity overall separation factor which can be determined from the binary overall separation factor ${ }^{2}$ as

$$
\gamma_{u}=\gamma^{(238-235)^{-1}}=\gamma^{1 / 3}
$$

Equation (5.1) is a well-known empirical relationship that has shown to be in agreement with both computational and experimental results [98]. Additionally, the sum of the isotopes in the mixture must equal one and the following condition is made

$$
\sum_{i=1}^{J} N_{i}^{\prime}=\sum_{i=1}^{J} N_{i}^{\prime \prime}=1
$$

The solution procedure for the multicomponent productivity analysis is an iterative

\footnotetext{
${ }^{1}$ These values are specified in ASTM C787-06 Standard Specification for Uranium Hexafluoride for Enrichment found in Nuclear Material Safeguards for Uranium Enrichment Plants [8].

${ }^{2}$ This is the separation factor between ${ }^{235} \mathrm{U}$ and ${ }^{238} \mathrm{U}$ and is typically displayed in a performance map.
} 
process [97] beginning with an initial guess for the cascade product concentrations. The initial guess is calculated by using the ideal multicomponent gradient solution [97].

Consider a countercurrent, one-up, one-down cascade with $n=n_{E}$ stages in the enriching section of the cascade and $m=m_{S}$ stages in the stripping section. The initial guess for the cascade product concentration can be calculated by

$$
\left(N_{P, i}\right)_{G}=\frac{\left(P N_{P, i}\right)_{G}}{\sum_{i=1}^{J}\left(P N_{P, i}\right)_{G}}
$$

where

$$
\left(P N_{P, i}\right)_{G}=N_{F, i} \cdot\left[1+\frac{1-\bar{\gamma}_{i}^{-n_{E}}}{\bar{\gamma}_{i}^{\left(m_{S}+1\right)}-1}\right]^{-1}
$$

and

$$
\bar{\gamma}_{i}=\frac{\gamma_{i, n_{E}}}{\sqrt{\gamma_{3, n_{E}}}}
$$

where the terms bracketed by ()$_{G}$ denote the initial guess [97].

Next, marching from the product stage of the cascade to the feed stage, the stage upflow concentrations in the enriching section of the cascade can be calculated by taking a material balance about the top stage of the cascade and the $\mathrm{n}^{\text {th }}$ stage for each component

$$
N_{E, n, i}^{\prime}=\frac{\left(L_{n}-P\right) N_{E, n+1, i}^{\prime \prime}+P N_{P, i}}{L_{n}}
$$

and the stage downflow concentrations can be calculated from the separation factors

$$
N_{E, n, i}^{\prime \prime}=\frac{N_{E, n, i}^{\prime}}{\gamma_{n, i}} \cdot\left[\sum_{i=1}^{J} \frac{N_{E, n, i}^{\prime}}{\gamma_{n, i}}\right]^{-1}
$$

Similarly for the stripping section of the cascade, marching from the bottom stage of the cascade to the feed stage $\left(m=m_{S}+1\right)$, the stages downflow concentrations can be 
calculated by taking a material balance about the bottom stage and the $\mathrm{m}^{\text {th }}$ stage

$$
N_{S, m, i}^{\prime \prime}=\frac{L_{m-1} N_{S, m-1, i}^{\prime}+W N_{W, i}}{L_{m-1}+W}
$$

and the stage upflow concentrations can be calculated from the separation factors

$$
N_{S, m, i}^{\prime}=\frac{N_{S, m, i}^{\prime \prime}}{\gamma_{n, i}} \cdot\left[\sum_{i=1}^{J} \frac{N_{S, m, i}^{\prime \prime}}{\gamma_{n, i}}\right]^{-1}
$$

At the feed stage of the centrifuge, a residual is calculated as

$$
\Delta_{i}=\left|N_{E, 1, i}^{\prime \prime}-N_{S, m_{S}+1, i}^{\prime \prime}\right|
$$

If the residual is smaller than a specified tolerance, the solution is considered converged. If not, a new guess is taken for the cascade product concentrations using a Newton-Raphson method and the solution procedure of Equations (5.7) - (5.10) is repeated. This calculation fully defines the multicomponent concentrations in the normal operation of the designed cascade.

The multicomponent productivity analysis described above is performed using the subroutine $P C F X 4$ by Ed von Halle. PCFX4 is a fixed plant, four component, productivity program that calculates the cascade concentrations based on stage upflow rates and binary stage separation factors. For this work, PCFX4 was modified to include six components and ported to Matlab.

To study the quasi-transient behavior of the cascade, the flow rates can be incrementally reduced. This is done by specifying a flow-scale-factor which reduces the cascade product rate and stage upflow rates. For this work, it is assumed that the cascade feed rate remains constant in off-normal operation and the cascade tails rate varies to account for the change in the cascade product rate.

By reducing the stage upflow rates, the stage feed and downflow rates also change and are calculated by material balances across the stage and at the stage feed point. The 
Table 5.2: Iguaçu centrifuge optimal operating conditions

\begin{tabular}{lcc}
\hline Parameter & Unit & Value \\
\hline Feed Rate & $\mathrm{mgUF}_{6} / \mathrm{s}$ & 31.81 \\
Cut (fixed) & - & 0.5 \\
Wall Temperature Gradient & $\mathrm{K} / \mathrm{m}$ & 24.35 \\
Scoop Force & dynes & 854 \\
Separative Power & $\mathrm{SWU} / \mathrm{yr}$ & 4.34 \\
Separation Factor & - & 1.254 \\
\hline
\end{tabular}

centrifuge performance at the new feed rate is determined from the performance map and the new cascade concentrations are calculated by the multicomponent productivity analysis detailed in Equations (5.7)-(5.10).

\subsection{Results}

\subsubsection{Centrifuge Performance}

For this study, the Iguaçu centrifuge is chosen as the operating centrifuge with geometric parameters detailed in Table 2.3. As mentioned in Chapter 2, the Pancake code is used to characterize the separative performance of the centrifuge. For this study, the operating conditions are slightly different from the values used in the illustrative results in Chapter 2 and thus the predicted performance is slightly different. Additionally, the cut is held constant in the centrifuge performance analysis. The optimal operating conditions of the Iguaçu centrifuge used in this study are shown in Table 5.2.

In order to determine if the level of enrichment is due to a transient enrichment spike or intentional off-normal operation, two types of centrifuge performances are considered and two centrifuge performance maps are developed. In Case 1, the optimal design parameters (temperature gradient, and scoop force) are held constant over the range of centrifuge feed rates. In Case 2, the design parameters are re-optimized at each centrifuge feed rate. A noticeable difference can be see between the two performance maps shown in Figure 5.1. 


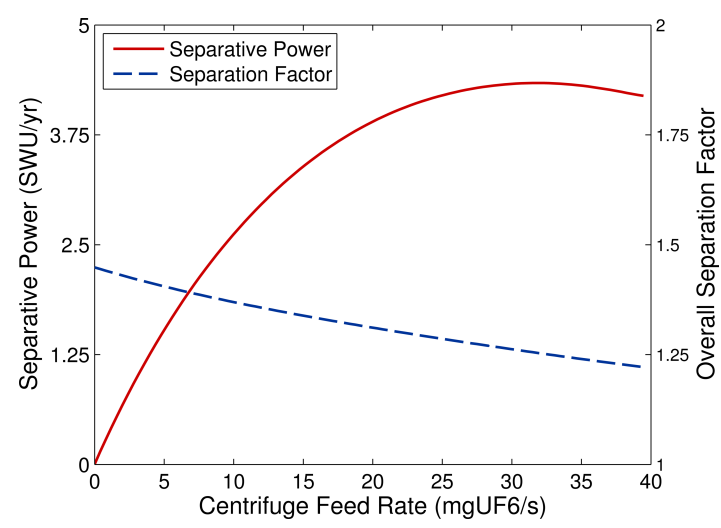

(a) Case 1

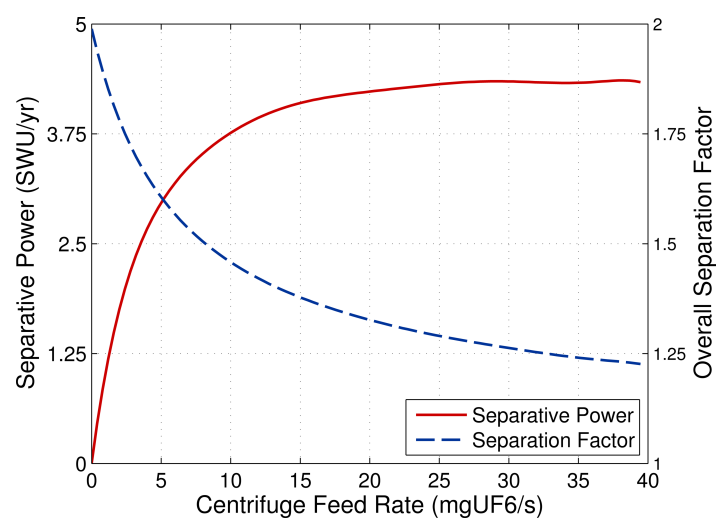

(b) Case 2

Figure 5.1: Performance maps for the Iguaçu centrifuge. Case 1 represents a normally operating centrifuge that has been optimized at the designed feed rate. Case 2 represents a centrifuge that is intentionally operated off-normally. At each feed rate, the scoop drag and temperature gradient are varied to optimize the centrifuge separative power at each feed rate.

For Case 2, the centrifuge separative power remains near the optimal value for a greater range of the centrifuge feed rate and the maximum separation factor $\left(\gamma_{\max }=\gamma\left(G_{c} \rightarrow 0\right)\right)$ is 1.98 compared with 1.45 for Case 1 . Case 1 represents the expected performance of the centrifuge during normal operation, whereas Case 2 represents intentional off-normal operation of the cascade by the operator. Case 2 can also be described as the "worst case scenario" in a proliferation analysis.

\subsubsection{Designed Cascade Normal Operation}

The cascade and normal operating conditions are determined from the procedure described in Section 7.2 by specifying nominal target parameters: $P=5000 \mathrm{kgU} / \mathrm{yr}, N_{P, 3}=0.050$, and $N_{W, 3}=0.00260$. The details of the cascade operating parameters are shown in Table 5.3. Because different enrichment plants can use different feed sources, the two types detailed in Table 5.1 are both considered. The cascade product concentrations for the normal operation of the designed cascade are shown in Table 5.4. 
Table 5.3: Designed cascade, normal operating parameters

\begin{tabular}{lcc}
\hline Parameter & Unit & Value \\
\hline Feed Rate & $\mathrm{kgU} / \mathrm{yr}$ & 50,198 \\
Product Rate & $\mathrm{kgU} / \mathrm{yr}$ & 5,000 \\
Tails Rate & $\mathrm{kgU} / \mathrm{yr}$ & 45,198 \\
Stages & - & $18 \mathrm{E}, 8 \mathrm{~S}, 26 \mathrm{~T}$ \\
Separative Power & $\mathrm{SWU} / \mathrm{yr}$ & 38,730 \\
Number of Centrifuges & - & 8,935 \\
\hline
\end{tabular}

Table 5.4: Designed cascade, normal operating product concentrations

\begin{tabular}{lcc}
\hline Isotope & $\begin{array}{c}\text { Natural Uranium } \\
\text { (at. frac.) }\end{array}$ & $\begin{array}{c}\text { Reprocessed Uranium } \\
\text { (at. frac.) }\end{array}$ \\
\hline${ }^{232} \mathrm{U}$ & $9.59 \times 10^{-11}$ & $4.79 \times 10^{-9}$ \\
${ }^{234} \mathrm{U}$ & $5.18 \times 10^{-4}$ & $1.83 \times 10^{-3}$ \\
${ }^{235} \mathrm{U}$ & $4.96 \times 10^{-2}$ & $8.36 \times 10^{-2}$ \\
${ }^{236} \mathrm{U}$ & $9.45 \times 10^{-5}$ & $1.96 \times 10^{-3}$ \\
\hline
\end{tabular}

In the off-normal operation of the cascade, four cases can be defined:

- Case ON: Case 1 (Optimized) Performance Map with Natural Feed

- Case OR: Case 1 (Optimized) Performance Map with Reprocessed Feed

- Case RN: Case 2 (Re-optimized) Performance Map with Natural Feed

- Case RR: Case 2 (Re-optimized) Performance Map with Reprocessed Feed

To determine the range of concentrations that may be present in the cascade due to a transient enrichment spike and by way of intentional off-normal operation, the flow-scalefactor is defined as

$$
\zeta=\frac{L_{n, \text { off-normal }}}{L_{n, \text { designed }}}
$$

Figure 5.2 shows the cascade product concentration of all the isotopes versus the flowscale-factor (also called the scaled product rate). The figure shows several trends for all the isotopes, namely, the product is most enriched in Case RR and least enriched in Case ON as expected. The centrifuges of Case 1 and Case 2 perform equally in the $\zeta=0.6-1.0$ range, 
below this the effect of re-optimizing the centrifuge is clear and leads to a large difference in enrichment.

When comparing measured environmental sampling results to that predicted by the model, as the flow-scale-factor is reduced to zero, Cases ON and OR represent the maximum value that should be seen in the cascade due to cascade startup depending on the type of material feed into the cascade. Cases RN and RR represent cases where the operator may be intentionally misusing the cascade.

In terms of proliferation capabilities, when considering the fissile isotope $\left({ }^{235} \mathrm{U}\right)$, the maximum potential of the cascade to produce the most enrichment at the highest throughput is determined by Case RR. For instance, reducing the flow-scale-factor to 0.2 , the cascade can produce product near $25 \%{ }^{235} \mathrm{U}$ at a rate of $1,000 \mathrm{kgU} / \mathrm{yr}$ which is roughly $1 \mathrm{SQ}$ of HEU material.

When analyzing environmental sampling results, the concentration of the minor isotopes $\left({ }^{232} U,{ }^{234} U,{ }^{236} U\right)$ are often compared with the concentration of ${ }^{235} U$ [17]. Bush et. al. compare the sampling results with expected values determined by

$$
N_{P, i}=\frac{N_{F, i}}{N_{F, j}} \cdot N_{P, j}
$$

This equation plots a straight line with the slope of the line equal to the ratio of the feed concentrations of the two isotopes. The line provides a fair estimate, but does not seem to follow the trend of the sampling results (see Figure 1 in [17]). From the sampling data, the upward facing parabolic trend in the ${ }^{234} U$ results show that the concentration of ${ }^{234} U$ increases 'faster' than ${ }^{235} \mathrm{U}$. The opposite trend is found for ${ }^{236} \mathrm{U}$. Figures 5.3 and ?? show the ratios of ${ }^{234} \mathrm{U}$ and ${ }^{236} \mathrm{U}$ versus ${ }^{235} \mathrm{U}$ respectively. These figures show that the model developed in this chapter predicts the same trends, that is, the greater relative enrichment for ${ }^{234} \mathrm{U}$ and the lower relative enrichment for ${ }^{236} \mathrm{U}$.

These trends can be explained by considering the mass difference between the isotopes and the characteristics of the gas centrifuge. Examining Equation (5.1), it can be seen that 

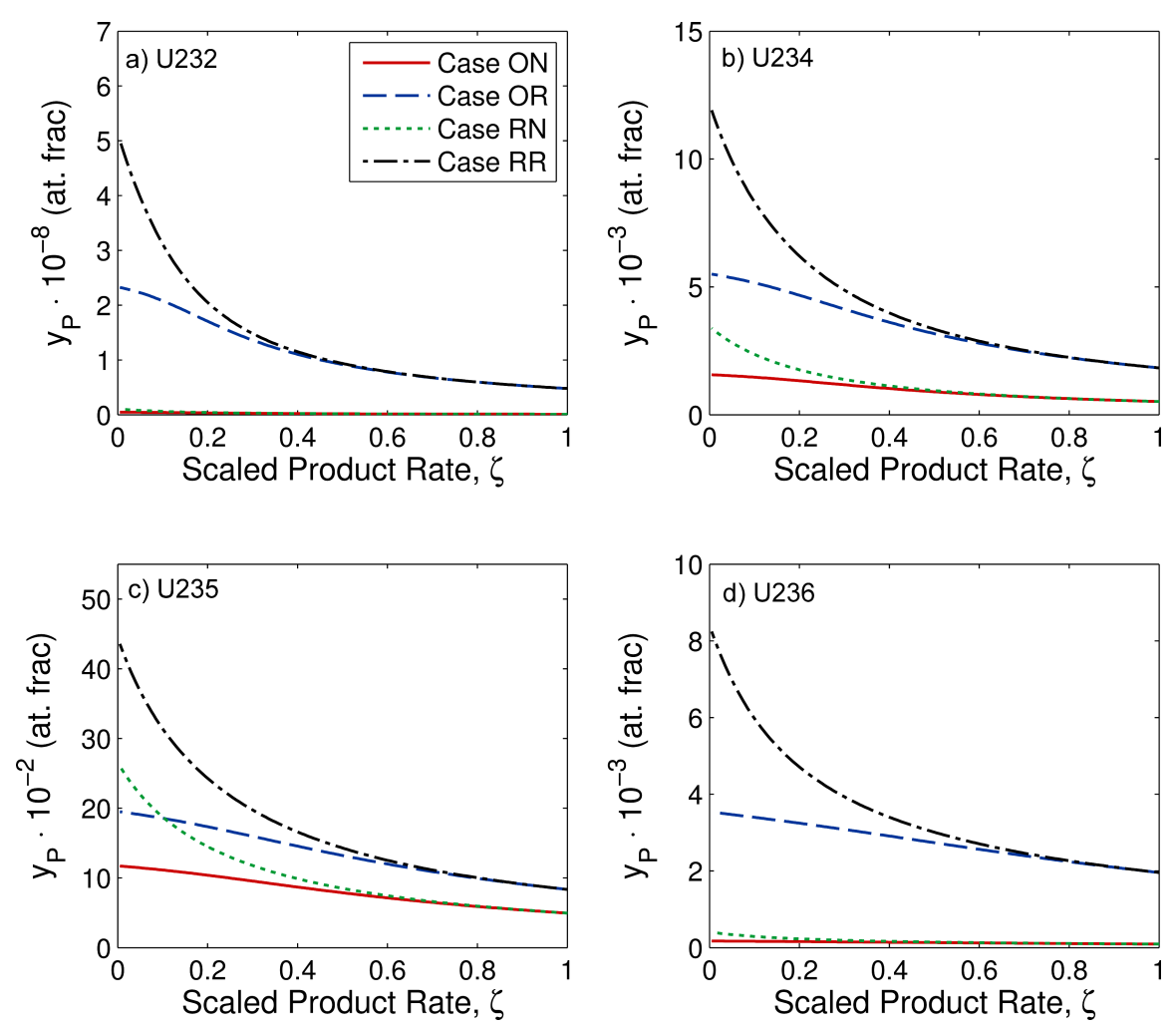

Figure 5.2: Cascade product concentrations versus the scaled product rate for: a) ${ }^{232} U, b$ ) ${ }^{234} \mathrm{U}$, c) $\left.{ }^{235} \mathrm{U}, \mathrm{d}\right){ }^{236} \mathrm{U}$. Cases $\mathrm{ON}$ and OR represent the range of enrichments that can be achieved in a normally operating cascade. The maximum capability of a cascade that has not been reconfigured are represented in Cases $\mathrm{RN}$ and $\mathrm{RR}$.

$\gamma_{2}>\gamma_{3}>\gamma_{4}$. Thus, due to the strong centrifugal field imparted on the gas, the lighter the isotope, the 'faster' it will enrich. Additionally, the effect of centrifuge optimization does not become prominent until higher enrichments are achieved. This indicates that comparing the relative ratios of isotopes may not be the best manner to determine if a cascade is being misused or not.

\subsection{Summary}

In this chapter, a method for determining the range of enrichments that may be present in a cascade is detailed. Studying the effect of centrifuge optimization is used to show the potential differences between cases where over-enrichment is due to a transient enrichment spike or intentional misuse. For a real cascade, this model can be compared with measured 


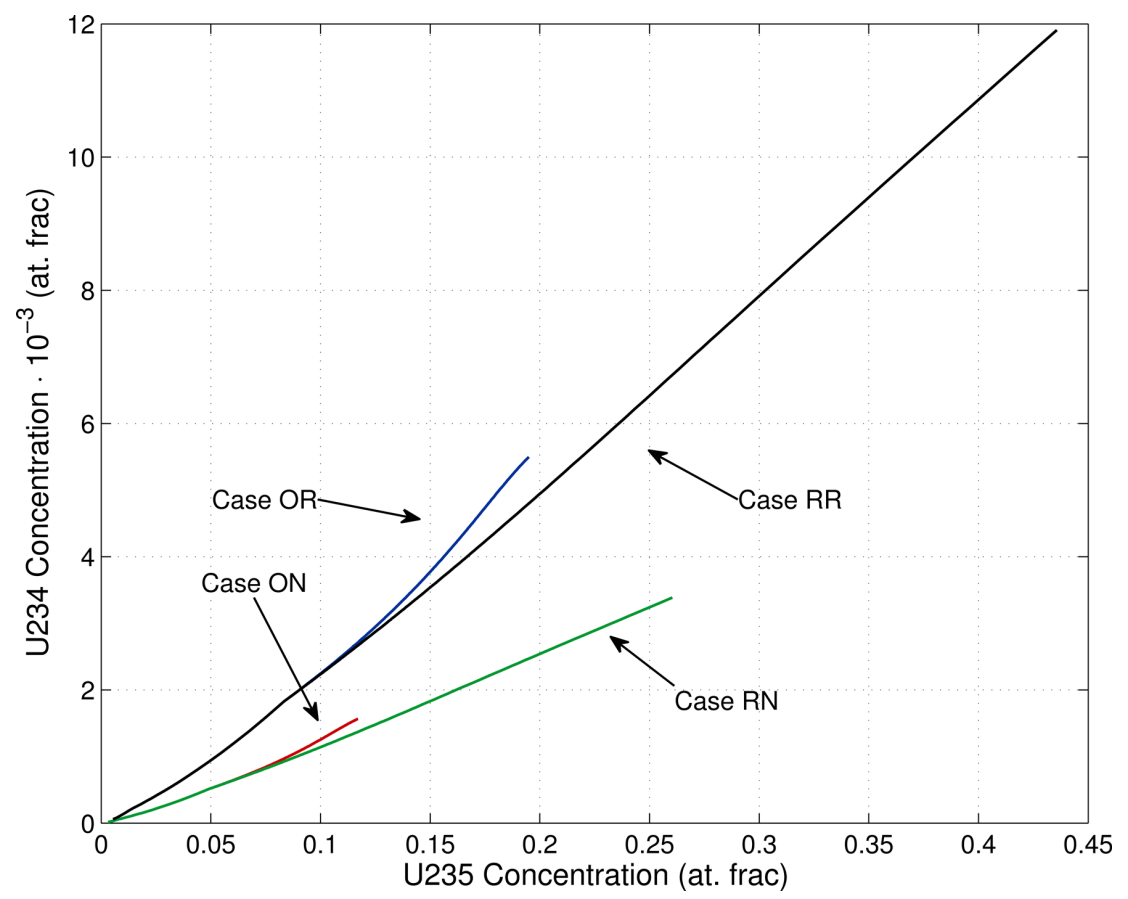

Figure 5.3: ${ }^{234} \mathrm{U}$ concentration versus ${ }^{235} \mathrm{U}$ concentration over the entire range of concentrations achievable. Because the ${ }^{234} U$ is lighter than ${ }^{235} U$, the ${ }^{234} U$ tends to enrich 'faster' than the ${ }^{235} \mathrm{U}$.

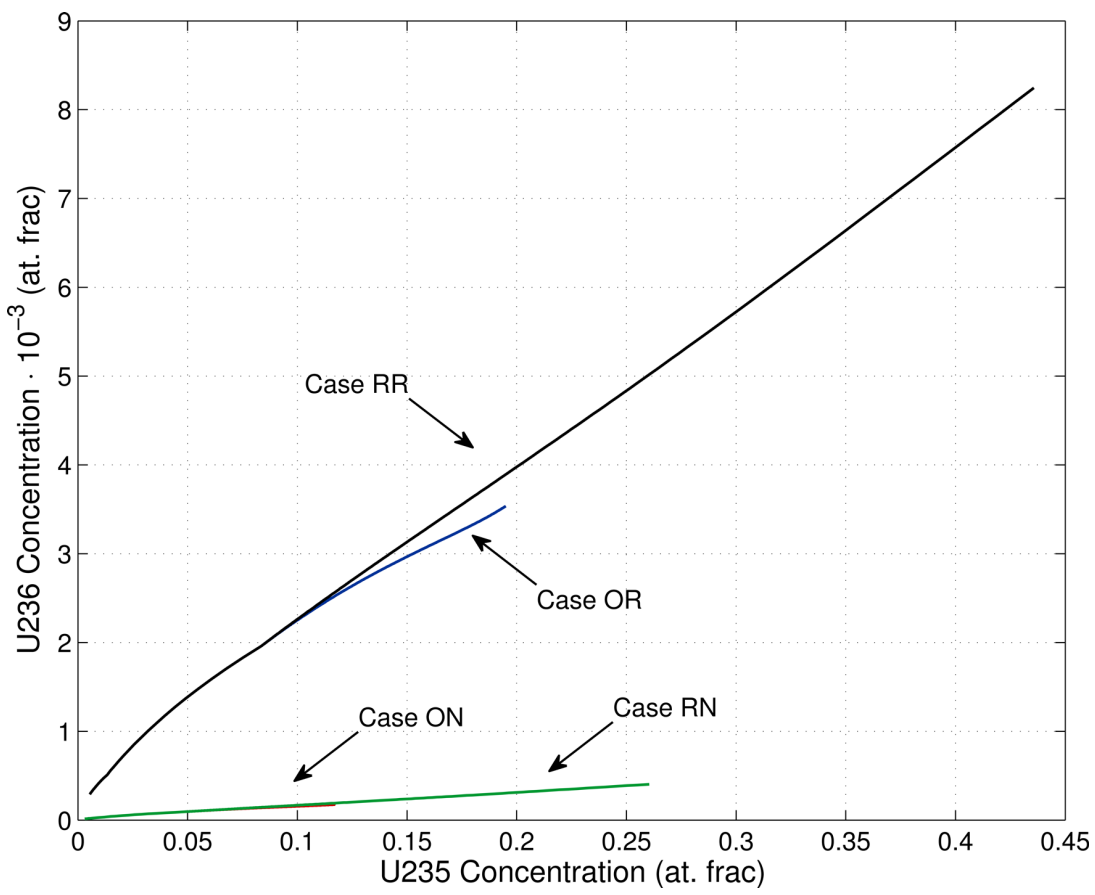

Figure 5.4: ${ }^{236} \mathrm{U}$ concentration versus ${ }^{235} \mathrm{U}$ concentration over the entire range of concentrations achievable. Because the ${ }^{236} \mathrm{U}$ is lighter than ${ }^{235} \mathrm{U}$, the ${ }^{236} \mathrm{U}$ tends to enrich 'slower' than the ${ }^{235} \mathrm{U}$. 
enrichment levels for a given cascade, allowing IAEA inspectors and analysts to decide whether a discrepancy is reasonable or not.

In order to make an assessment of what concentrations in the centrifuge plant are reasonable requires knowledge of the composition of the cascade feed. For a declared GCEP, information from the Design Information Verification would be a way to estimate a cascade design and operating parameters. The knowledge gained from using this model can help reduce the number of on-site inspection activities necessary and reduce the number of false alarms created by anomalies and resulting investigations. 


\section{Chapter 6}

\section{Semi-Empirical Method of Calculating a Centrifuge Performance Map}

\subsection{Overview}

As mentioned in Chapter 2, centrifuge performance maps detail the separative performance of a gas centrifuge over a range of operating parameters, usually feed rate and machine cut. Typically, a performance map is developed by modeling the internal hydrodynamics and isotope diffusion in the centrifuge and then simulating over a range of operating conditions. These models require a detailed knowledge of the geometry and features of a particular gas centrifuge including the rotational speed, length, radius, scoop geometry, baffle geometry, feed location, feed rate, cut, etc. Due to the sensitive nature of centrifuge research and development, many of these parameters are unknown to the general public and one is forced to estimate many of these factors, introducing uncertainty.

In some cases, information about how a centrifuge is operating in a cascade can be inferred from cascade data reported by the IAEA. For example, Albright et. al. [99, 100] calculate the minimal average separative power of the IR- 1 centrifuge at the Natanz Fuel Enrichment Plant based on data obtained from the IAEA Implementation of the NPT Safeguards Agreement in the Islamic Republic of Iran report [101]. Additionally, based on knowledge of the cascade structure at the Natanz plant, product enrichment, and assuming the cascade operates close to ideal, one can infer an average overall separation factor for 
the centrifuge. The separative power and separation factor are enough to characterize the centrifuge for that particular design point, but in a nonproliferation breakout analysis, it is often the case that cascade calculations are made with the centrifuge operating with off-design flow rates. For these cases, performance maps are needed to characterize the centrifuge over a wide range of material flow rates and stage cuts.

In Chapter 2, the gradient equation for the gas centrifuge was found to be

$$
\frac{m^{2}+1}{2 m} S_{0} \frac{d \bar{N}}{d z}=\psi \bar{N}(1-\bar{N})-\frac{\tau-\tau^{*} \bar{N}}{m L_{0}}
$$

for which an alternate form [38] in the enriching section is

$$
S \frac{d \bar{N}}{d z}=\psi \bar{N}(1-\bar{N})-\frac{P}{L}\left(N_{p}-\bar{N}\right)
$$

where $S$ is the stage length in the centrifuge. This form is quite similar to the approximate form of the cascade gradient equation.

In the gradient equation for the countercurrent gas centrifuge, the separation terms $\psi$ and $L$ that characterize the solution are functions of the axial mass flux in the centrifuge. To determine the axial mass flux, one must first solve the fluid dynamics governing equations. In lieu of calculating these parameters, one could assume values and treat the centrifuge as a cascade, solving the exact gradient equations for a range of feed rates and cuts to develop a performance map. In this chapter, the methodology will be developed for this treatment and the model with be verified by comparing to separation results from the Pancake code. A large portion of this chapter has been submitted to Separation Science and Technology under the title "Semi-Empirical Method for Developing a Centrifuge Performance Map." 


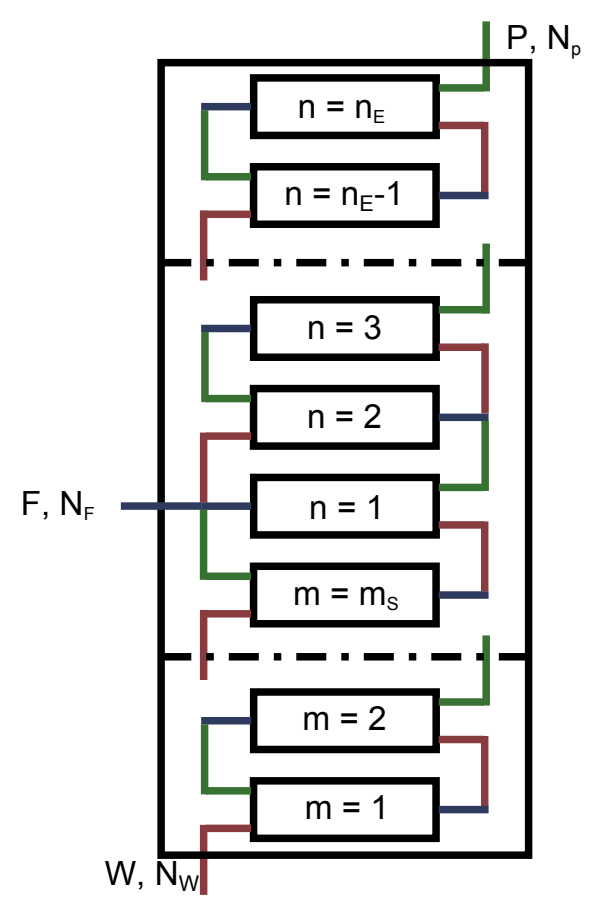

Figure 6.1: Cascade flow diagram for a theoretical cascade model of a gas centrifuge. The theoretical cascade is characterized by a number of stages, stage upflow rate, and stage enrichment factor.

\subsection{Methodology}

The centrifuge is assumed to behave like a countercurrent, symmetric cascade consisting of an enriching section with $n_{E}$ stages and a stripping section with $m_{S}$ stages (see Figure $6.1)$.

The stage concentrations in the centrifuge are calculated by solving the exact gradient equations for a general cascade (see Chapter 3) as a function of the stage upflow rates, stage enrichment factors, number of stages, centrifuge feed concentration, and two of the three centrifuge external flow rates. It is assumed that the centrifuge feed concentration is natural uranium and in order to calculate the centrifuge performance map, a range for the centrifuge feed rate and centrifuge cut are prescribed. The centrifuge product rate can then be calculated as 


$$
P_{c}=\theta_{c} F_{c}
$$

where $P_{c}$ is the centrifuge product rate, $\theta_{c}$ is the centrifuge cut, and $F_{c}$ is the centrifuge feed rate.

To simplify the analysis, it is assumed that the centrifuge operates as a square, general cascade. A square cascade is one where each stage in the cascade has the same feed rates. It is also assumed that the cut in each stage is constant, so that the stage upflow rates and stage enrichment factors are the same in each stage aside from the top stage. In the top stage, the stage upflow rate is equal to the centrifuge product rate. This is written as

$$
\begin{aligned}
& L_{E, n}= \begin{cases}L_{c} & : n<n_{E} \\
P_{c} & : n=n_{E}\end{cases} \\
& L_{S, m}=L_{c} \\
& \psi_{E, n}=\psi_{c} \\
& \psi_{S, m}=\psi_{c}
\end{aligned}
$$

This leaves three parameters to characterize the performance of the centrifuge at each feed rate and cut: the stage upflow rate $\left(L_{c}\right)$, the stage enrichment factor $\left(\psi_{c}\right)$, and the number of stages in the centrifuge $\left(n_{c}=n_{E}+m_{S}\right)$, assuming that the feed is introduced in the middle of the centrifuge.

The performance map is generated by first specifying two target separation parameters, the optimal separative power $\left(\delta U_{0 T}\right)$ and the optimal separation factor $\left(\gamma_{0 T}\right)$. Next, one of the three characterizing centrifuge parameters $\left(L_{c}, \psi_{c}, n_{c}\right)$ is fixed and an iterative procedure is used to find the value of the other two characterizing parameters that yield a solution that is approximately the target parameters. In this study, the number of stages in the centrifuge is fixed and the other two parameters are varied. This procedure is further illustrated in 


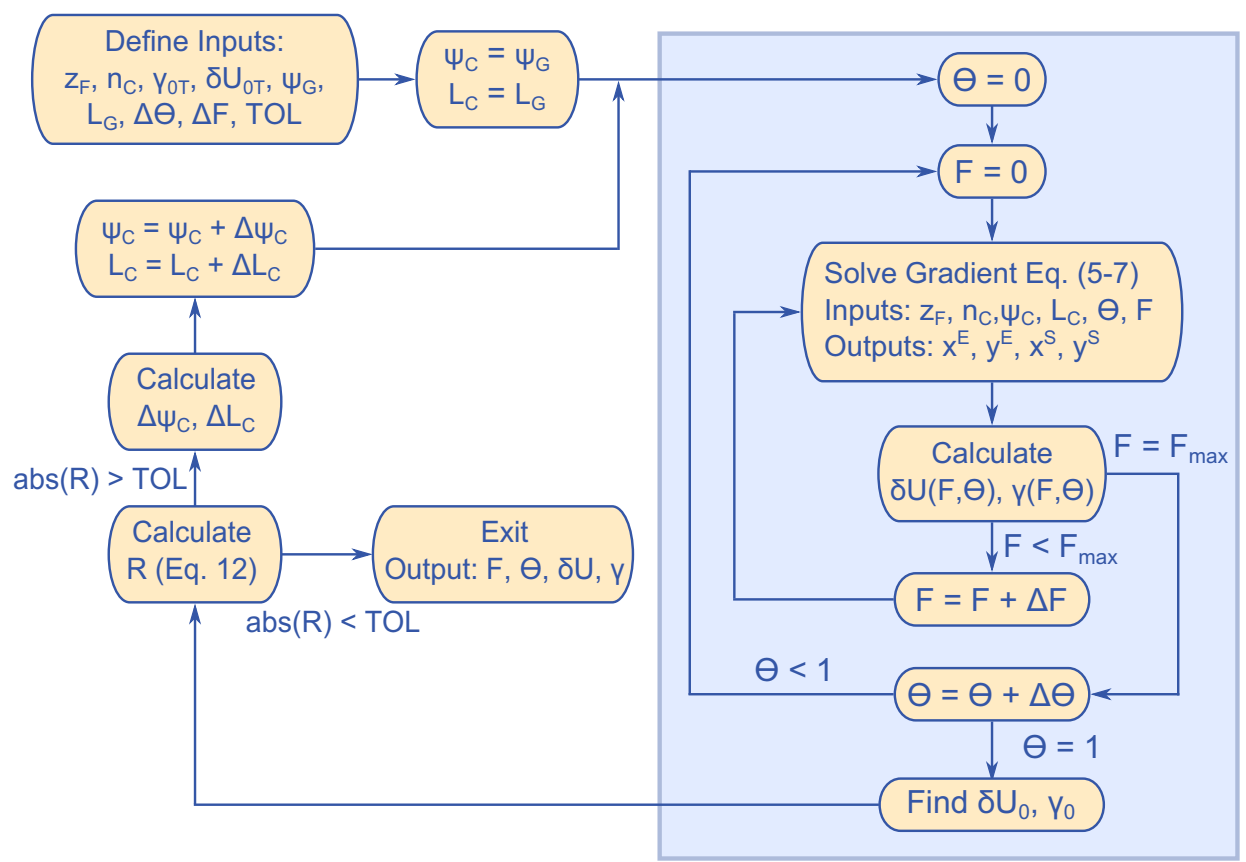

Figure 6.2: Algorithm flow chart for calculating centrifuge performance by treating the centrifuge as a square cascade. Target separation parameters are sought through an iterative procedure by solving the exact gradient equations over a range of feed rates and cuts.

Figure 6.2.

Referring to Figure 6.2, once the inputs are defined, three nested loops are used to calculate the optimal separative power and optimal separation factor. An initial guess is set for the stage upflow rate and stage enrichment factor and the gradient equations are solved over a range of cuts and feed rates to determine the centrifuge separative power and separation factor. Next, the optimal design point is found as the feed rate and cut where the separative power is maximized. The separative power and separation factor at this design point are deemed the optimal values. Two residuals are calculated as the percent difference between the calculated optimal values and the target values as

$$
\begin{aligned}
& R_{1}=\frac{\gamma_{0}-\gamma_{0 T}}{\gamma_{0 T}} \\
& R_{2}=\frac{\delta U_{0}-\delta U_{0 T}}{\delta U_{0 T}}
\end{aligned}
$$


A Newton-Raphson approach is used to iterate on $\psi_{c}$ and $L_{c}$ until both residuals are below a set value, then the program outputs the solution which contains the feed range, cut range, separative power, separation factor, and the stage upflow rate and stage enrichment factor that characterize that separative performance.

\subsection{Results}

In this section, illustrative results are presented and the method is verified by comparing to results from the Pancake code. First, comparisons are made at the centrifuge level for the separative power and separation factor over a range of feed rates and cuts. Next, a sample nonproliferation analysis is performed to compare the two models at the cascade level.

\subsubsection{Case Studies}

For this study, the Iguaçu centrifuge is chosen and optimal separation parameters calculated by the Pancake code are used as the target parameters. As shown in Chapter 2, the optimal parameters are, $\delta U_{0}=4.4082$ and $\gamma_{0}=1.27430$ at a feed rate of $28.22 \mathrm{mgUF}_{6} / \mathrm{s}$ and a cut of 0.470 .

Since the number of stage is fixed and must be arbitrarily chosen, it is prudent to study the effect that the number of stages has on the calculated characterizing parameters and the predicted centrifuge performance. Ten cases with different $n_{c}$ are chosen and the performance is calculated with a residual of $1 \mathrm{E}-4$. The cases and results for the optimal design points are shown in Table 6.1. The optimal design point predicted by the semiempirical model presented in here compares well with results from the Pancake code having a maximum percent difference of all cases of $0.860 \%$ for the optimal feed rate and $8.511 \%$ for the optimal cut. There are a few trends that are clear from these results:

- As the number of stages $\left(n_{c}\right)$ increases, the stage upflow rate $\left(L_{c}\right)$ increases linearly and the stage enrichment factor $\left(\psi_{c}\right)$ decreases exponentially. 
Table 6.1: Case summaries and calculated optimal design points

\begin{tabular}{|c|c|c|c|c|c|c|c|c|c|}
\hline \multirow[b]{2}{*}{ Case } & \multicolumn{3}{|c|}{$\begin{array}{c}\text { Calculated Characterizing } \\
\text { Parameters }\end{array}$} & \multicolumn{4}{|c|}{$\begin{array}{c}\text { Calculated Optimal } \\
\text { Parameters }\end{array}$} & \multicolumn{2}{|c|}{$\begin{array}{l}\% \text { Diff. from } \\
\text { Pancake }(\%)\end{array}$} \\
\hline & $n_{C}$ & $\psi_{C}$ & $L_{C}$ & $\gamma_{0}$ & $\Delta U_{0}$ & $F_{0}$ & $\theta_{0}$ & $F_{0}$ & $\theta_{0}$ \\
\hline $\mathrm{P}$ & - & - & - & 1.2743 & 4.4082 & 28.220 & 0.470 & - & - \\
\hline 1 & 10 & 0.04364 & 54.38 & 1.2743 & 4.4081 & 28.233 & 0.457 & 0.046 & 2.766 \\
\hline 2 & 14 & 0.03095 & 76.95 & 1.2743 & 4.4083 & 28.214 & 0.461 & 0.021 & 1.915 \\
\hline 3 & 15 & 0.02885 & 82.73 & 1.2743 & 4.4086 & 28.463 & 0.430 & 0.860 & 8.511 \\
\hline 4 & 16 & 0.02703 & 88.20 & 1.2743 & 4.4083 & 28.210 & 0.462 & 0.037 & 1.702 \\
\hline 5 & 20 & 0.02155 & 110.69 & 1.2743 & 4.4084 & 28.205 & 0.464 & 0.054 & 1.277 \\
\hline 6 & 35 & 0.01225 & 194.96 & 1.2743 & 4.4081 & 28.261 & 0.452 & 0.146 & 3.830 \\
\hline 7 & 50 & 0.00856 & 278.92 & 1.2743 & 4.4080 & 28.191 & 0.468 & 0.104 & 0.426 \\
\hline 8 & 51 & 0.00839 & 284.66 & 1.2743 & 4.4082 & 28.228 & 0.458 & 0.029 & 2.553 \\
\hline 9 & 80 & 0.00534 & 446.83 & 1.2743 & 4.4078 & 28.177 & 0.469 & 0.154 & 0.213 \\
\hline 10 & 100 & 0.00427 & 559.12 & 1.2743 & 4.4086 & 28.191 & 0.469 & 0.104 & 0.213 \\
\hline
\end{tabular}

- When comparing cases with similar number of stages (i.e. $n_{c}=50$ vs. $n_{c}=51$ ), cases where the number of stages is even are in better agreement with Pancake for the predicted optimal cut.

- Among cases where the $n_{c}$ is even, the agreement in predicted optimal cut between

Pancake and the semi-empirical method increases as the number of stages increases.

There are no clear trends between the number of stages chosen in the centrifuge and the agreement in predicted optimal feed rate.

\subsubsection{Centrifuge Level Comparison}

Figure 6.3 shows the separative power versus feed rate for all cases compared with results from Pancake for a range of cuts. Table 6.2 shows the percent differences between each cases and results from Pancake averaged over all feed rates for the predicted separative power. Overall, there is good agreement amongst each of the cases and fairly good agreement between the cases and results from Pancake with average percent difference in the range of $14-19 \%$ for all feed rates and cuts. Case 10 shows a marginally better agreement with Pancake the other cases. All cases show very good agreement with Pancake 
in the $0-20 \mathrm{mgUF}_{6} / \mathrm{s}$ feed rate range for all centrifuge cuts. Typically, most centrifuges operate with a cut near 0.50 for which both models are in good agreement, within $5 \%$.

Figure 6.4 shows the overall separation factor versus feed rate for all cases compared with results from Pancake for a range of cuts. Table 6.3 shows the percent differences between each case and results from Pancake averaged over all feed rates for the predicted separation factor. There is very little difference between the each case and for most cuts the agreement between the cases and Pancake is very good with average percent differences less than $4 \%$ for all cuts and less than $1 \%$ for cuts greater than 0.275 . The excellent agreement shown between the two models for the separation factor is especially important because this is the separation factor that is typically used in cascade analyses.

\subsubsection{Cascade Level Comparison}

In order to determine what effect the differences in predicted performance between the semi-empirical method and Pancake have on a nonproliferation study, a cascade analysis is performed for a designed cascade and several off-design cases. The designed cascade is determined by squaring-off an ideal cascade with a nominal capacity of 50,000 SWU/yr, a product enrichment of $5 \%{ }^{235} U$, and natural uranium feed. For the designed cascade, the performance map of the Iguaçu machine predicted by Pancake is used to determine the stage separation factor as a function of feed rate and cut. The details of the designed cascade are shown in Table 6.4. The cascade is slightly less efficient than the nominal due to mixing effects.

To develop the off-design cascade operation, flow-scale-factor defined in Chapter 5 is used to reduce the stage upflow rates and cascade product rate while keeping the cascade feed rate constant. The original feed rates and downflow rates in each stage of the cascade are adjusted according to the flow-scale-factor and then the stage separation factors are determined by the performance map for each of the $n_{c}$ cases. The stage concentrations are calculated by performing a productivity analysis (see Chapter 3). 

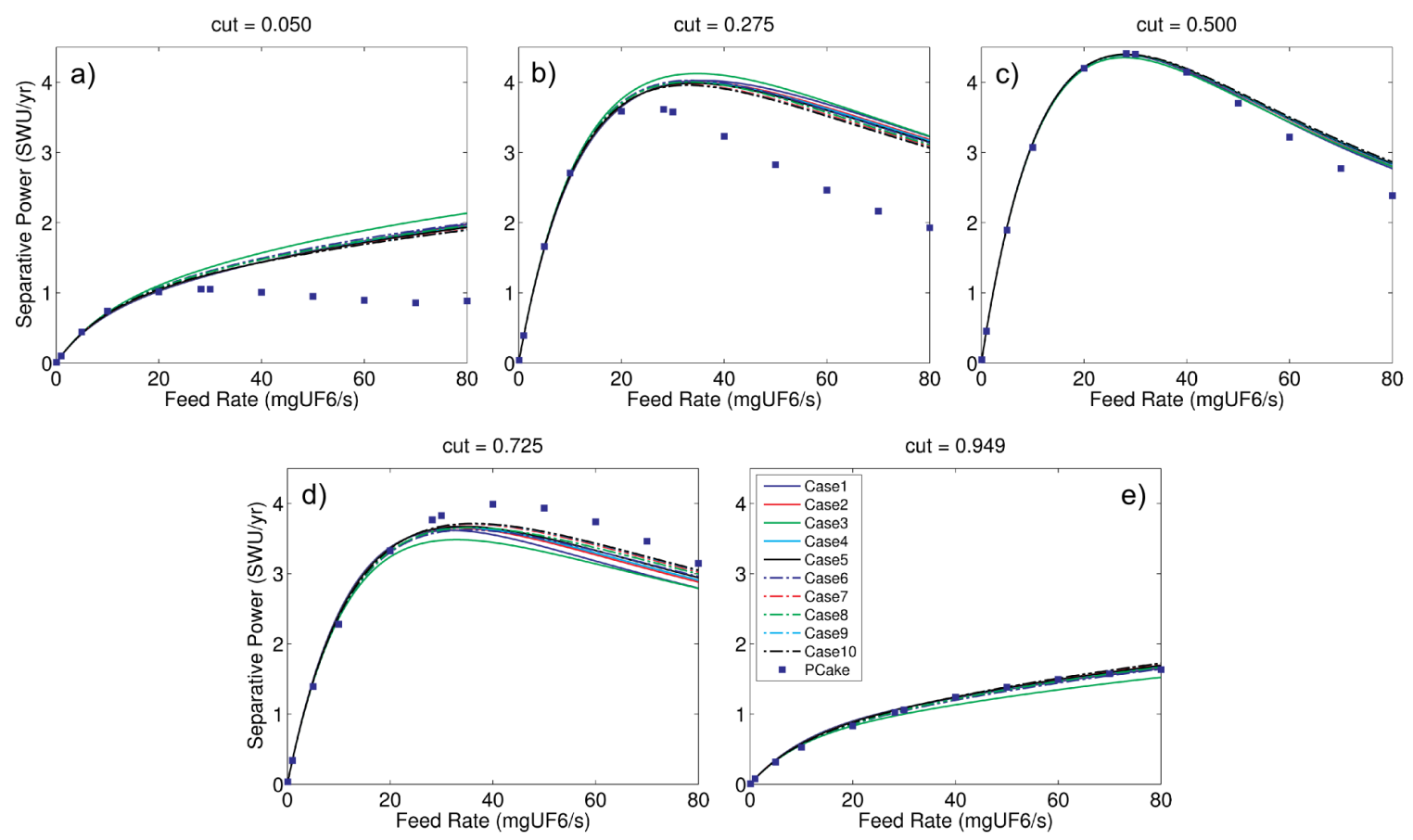

Figure 6.3: Comparison of the predicted centrifuge separative power versus feed rate for: a) $\theta=0.05$, b) $\theta=0.275$, c) $\theta=0.50$, d) $\theta=0.725$, e) $\theta=0.949$. Good agreement is found between the semi-empirical model and Pancake for typically operating conditions $(\theta=0.50)$. Over the entire range, the average percent difference is $14.25-18.30 \%$.

Table 6.2: Percent differences between each case and Pancake averaged overall all feed rates for the predicted separative power (in \%)

\begin{tabular}{|c|c|c|c|c|c|c|c|c|c|c|}
\hline \multirow[b]{2}{*}{ Cut } & \multicolumn{10}{|c|}{ Case } \\
\hline & 1 & 2 & 3 & 4 & 5 & 6 & 7 & 8 & 9 & 10 \\
\hline 0.050 & 41.61 & 41.05 & 49.93 & 40.86 & 40.58 & 43.87 & 39.82 & 42.43 & 39.62 & 39.55 \\
\hline 0.275 & 22.19 & 21.38 & 23.31 & 21.11 & 20.71 & 20.99 & 19.68 & 20.38 & 19.38 & 19.32 \\
\hline 0.500 & 3.68 & 4.15 & 3.90 & 4.27 & 4.39 & 4.43 & 4.57 & 4.51 & 4.58 & 4.61 \\
\hline 0.725 & 7.72 & 6.58 & 8.49 & 6.25 & 5.80 & 5.86 & 4.78 & 5.27 & 4.55 & 4.46 \\
\hline 0.950 & 4.08 & 3.63 & 5.87 & 3.54 & 3.44 & 2.94 & 3.32 & 2.59 & 3.33 & 3.31 \\
\hline All & 15.86 & 15.36 & 18.30 & 15.20 & 14.98 & 15.62 & 14.43 & 15.04 & 14.29 & 14.25 \\
\hline
\end{tabular}



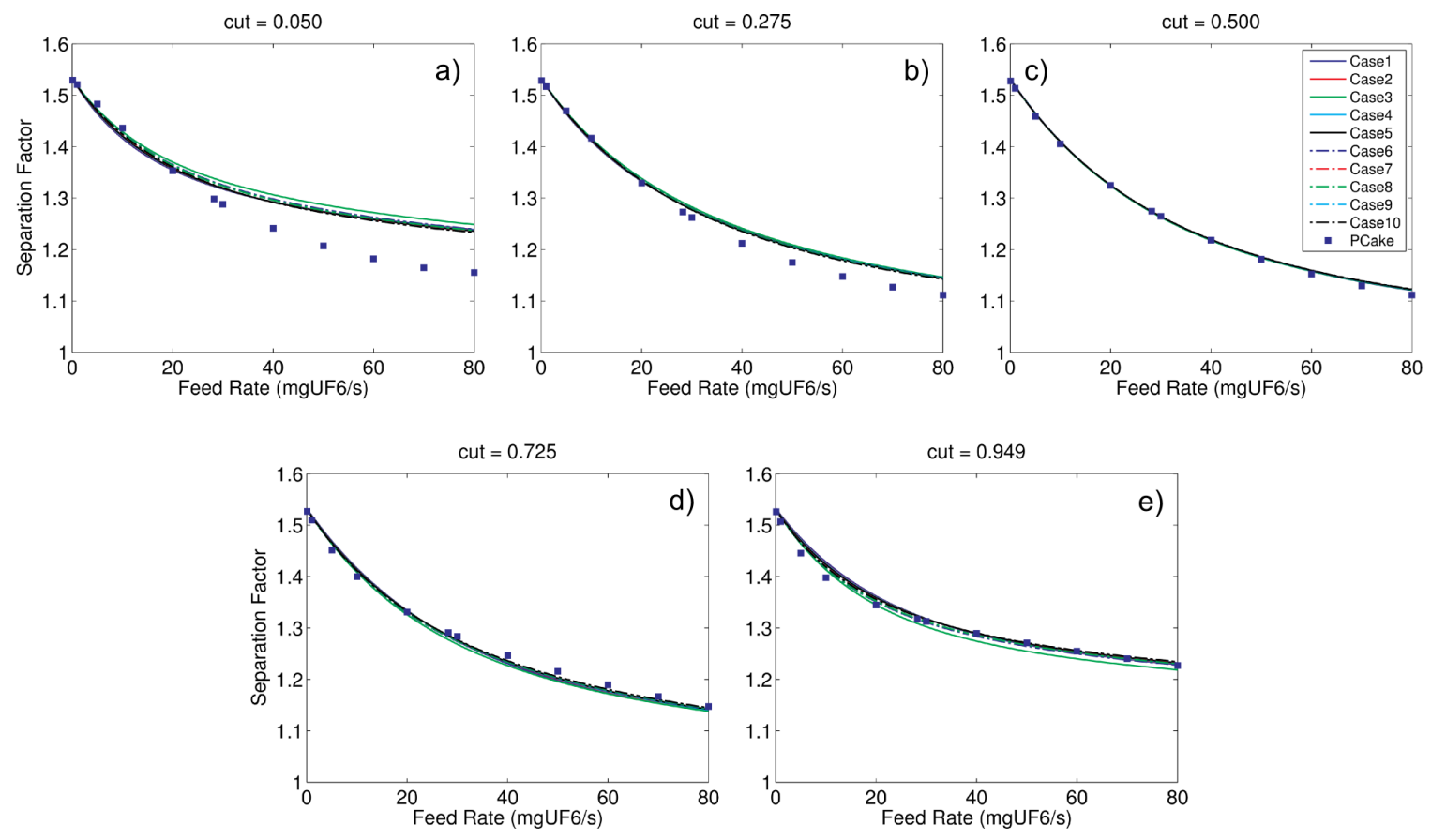

Figure 6.4: Comparison of the predicted centrifuge separation factor versus feed rate for: a) $\theta=0.05$, b) $\theta=0.275$, c) $\theta=0.50$, d) $\theta=0.725$, e) $\theta=0.949$. Very good agreement is found between the two models for the entire range of operating conditions. The average percent difference is $1.15-1.49 \%$.

Table 6.3: Percent differences between each case and Pancake averaged overall all feed rates for the predicted separation factor (in \%)

\begin{tabular}{ccccccccccc}
\hline & \multicolumn{1}{c}{ Case } \\
Cut & 1 & 2 & 3 & 4 & 5 & 6 & 7 & 8 & 9 & 10 \\
\hline 0.050 & 3.17 & 3.13 & 3.74 & 3.11 & 3.09 & 3.32 & 3.04 & 3.22 & 3.02 & 3.02 \\
0.275 & 1.50 & 1.44 & 1.58 & 1.42 & 1.40 & 1.42 & 1.32 & 1.37 & 1.30 & 1.30 \\
0.500 & 0.31 & 0.33 & 0.31 & 0.34 & 0.34 & 0.33 & 0.34 & 0.34 & 0.34 & 0.34 \\
0.725 & 0.90 & 0.78 & 0.95 & 0.74 & 0.69 & 0.68 & 0.58 & 0.62 & 0.56 & 0.54 \\
0.950 & 0.71 & 0.63 & 0.84 & 0.61 & 0.59 & 0.48 & 0.54 & 0.44 & 0.54 & 0.53 \\
All & 1.32 & 1.26 & 1.49 & 1.25 & 1.22 & 1.25 & 1.17 & 1.20 & 1.15 & 1.15 \\
\hline
\end{tabular}


Table 6.4: Design cascade based on Pancake performance map

\begin{tabular}{lcc}
\hline Parameter & Unit & Value \\
\hline Feed Rate & $\mathrm{kgU} / \mathrm{yr}$ & 61,404 \\
Product Rate & $\mathrm{kgU} / \mathrm{yr}$ & 5,692 \\
Tails Rate & $\mathrm{kgU} / \mathrm{yr}$ & 55,712 \\
Feed Concentration & at. frac. & 0.00711 \\
Product Concentration & at. frac. & 0.05319 \\
Tails Concentration & at. frac. & 0.00240 \\
Stages & - & $17 \mathrm{E}, 8 \mathrm{~S}, 25 \mathrm{~T}$ \\
Centrifuges & - & 11,329 \\
Separative Power & $\mathrm{SWU} / \mathrm{yr}$ & 49,943 \\
\hline
\end{tabular}

The comparison of the design and off-design operation between the different cases and Pancake are shown in Table 6.5. In the table, the percent differences from Pancake are shown for the cascade product concentration, the cascade tails concentration, and the separative power of the cascade. The results show very good agreement between all of the cases and Pancake for all parameters with the cascade product concentration being in the best agreement. The largest discrepancies that are found in cascade level study are for the 2.0 flow scale factor and are due to the increased centrifuge flow rate where the differences between the two models are greatest. Flow-scale-factors of this magnitude are rather extreme and are typically not considered. For instance, Witt et. al. [102] restricted the flow scale factor in a range of $0.95-1.05$ for which the difference between the two models is less than $1 \%$.

\subsection{Summary}

In this paper, a semi-empirical method for developing a centrifuge performance map based on knowledge of the machine's operating separative power and separation factor is detailed. This type of information can be inferred from IAEA reports and is used as target parameters for the model. In order to verify the model, separation results are compared to results from the Pancake code at both the centrifuge and cascade level. 
Table 6.5: Percent difference between all cases and Pancake for the predicted cascade product concentration, cascade tails concentration, and separative power

\begin{tabular}{|c|c|c|c|c|c|c|c|c|c|c|c|}
\hline \multirow{3}{*}{$\begin{array}{l}\text { Scale } \\
\text { Factor }\end{array}$} & \multirow{3}{*}{ Term } & \multicolumn{10}{|c|}{ Percent Difference from Pancake (\%) } \\
\hline & & \multicolumn{10}{|c|}{ Case } \\
\hline & & 1 & 2 & 3 & 4 & 5 & 6 & 7 & 8 & 9 & 10 \\
\hline \multirow{3}{*}{0.1} & $N_{P}$ & 0.205 & 0.130 & 0.173 & 0.113 & 0.096 & 0.043 & 0.081 & 0.004 & 0.056 & 0.075 \\
\hline & $N_{W}$ & 0.042 & 0.027 & 0.036 & 0.023 & 0.020 & 0.009 & 0.017 & 0.001 & 0.012 & 0.016 \\
\hline & $\Delta U$ & 0.269 & 0.171 & 0.227 & 0.148 & 0.127 & 0.057 & 0.106 & 0.005 & 0.074 & 0.099 \\
\hline \multirow{3}{*}{0.8} & $N_{P}$ & 0.344 & 0.342 & 0.429 & 0.342 & 0.342 & 0.352 & 0.347 & 0.345 & 0.347 & 0.343 \\
\hline & $N_{W}$ & 0.690 & 0.686 & 0.860 & 0.686 & 0.685 & 0.706 & 0.696 & 0.692 & 0.696 & 0.687 \\
\hline & $\Delta U$ & 0.777 & 0.773 & 0.967 & 0.772 & 0.771 & 0.794 & 0.784 & 0.779 & 0.783 & 0.774 \\
\hline \multirow{3}{*}{1.0} & $N_{P}$ & 0.023 & 0.011 & 0.183 & 0.009 & 0.005 & 0.037 & 0.005 & 0.019 & 0.007 & 0.001 \\
\hline & $N_{W}$ & 0.052 & 0.025 & 0.415 & 0.020 & 0.012 & 0.083 & 0.011 & 0.042 & 0.015 & 0.001 \\
\hline & $\Delta U$ & 0.056 & 0.028 & 0.448 & 0.022 & 0.013 & 0.090 & 0.012 & 0.045 & 0.016 & 0.001 \\
\hline \multirow{3}{*}{1.2} & $N_{P}$ & 0.277 & 0.236 & 0.470 & 0.227 & 0.215 & 0.254 & 0.200 & 0.225 & 0.202 & 0.192 \\
\hline & $N_{W}$ & 0.678 & 0.578 & 1.151 & 0.556 & 0.526 & 0.622 & 0.491 & 0.551 & 0.495 & 0.471 \\
\hline & $\Delta U$ & 0.720 & 0.614 & 1.218 & 0.590 & 0.558 & 0.660 & 0.522 & 0.584 & 0.525 & 0.500 \\
\hline \multirow{3}{*}{2.0} & $N_{P}$ & 0.928 & 1.191 & 0.866 & 1.252 & 1.325 & 1.326 & 1.432 & 1.389 & 1.438 & 1.456 \\
\hline & $N_{W}$ & 2.309 & 2.962 & 2.154 & 3.113 & 3.295 & 3.299 & 3.562 & 3.456 & 3.577 & 3.621 \\
\hline & $\Delta U$ & 2.875 & 3.702 & 2.679 & 3.895 & 4.127 & 4.131 & 4.467 & 4.332 & 4.487 & 4.544 \\
\hline
\end{tabular}

For centrifuge performance prediction, the semi-empirical method agrees very well with Pancake, to within an average of less than $2 \%$ for the overall separation factor and near $15 \%$ for the separative power. It is found that the cases with the largest, even number of stages in the centrifuge show better agreement with Pancake.

At the cascade level, several off-design operation cases are presented to study the how the differences between the two models would affect a nonproliferation analysis. The results show that for the cascade product concentration, the semi-empirical method is in very good agreement with Pancake with percent differences below $2 \%$. The maximum difference between these two models is found in the most unrealistic scenario where the flow-scale-factor is set to 2.0 .

The results of this study show that the semi-empirical model can provide a good estimate of centrifuge performance over a broad range of feed rates and cuts based on very little information about the centrifuge. 


\section{Chapter 7}

\section{A Fixed Plant Method for Quantifying Breakout Times}

\subsection{Overview}

In the marginal chance that a State does withdraw from the NPT and wishes to pursue a nuclear weapon, it is imperative to accurately predict the capabilities of their enrichment program and determine the breakout time, the time it takes to achieve one SQ of WGU. For this study, the IAEA definition will be used, i.e. one SQ of WGU is $25 \mathrm{~kg}^{235} \mathrm{U}$ at $90 \%$ enrichment.

To estimate breakout time, analysts typically employ ideal separative work calculations [103]. In this type of analysis, a cascade hall is treated as a 'black box' (see Figure 7.1) and the separative power of all the centrifuges is lumped together, disregarding cascade structure. As an example, consider a production unit containing 10 cascades with 500 centrifuges each and these centrifuges with the capacity to produce $2 \mathrm{SWU} / \mathrm{yr}$. In the ideal case, the production unit will have a separative power of 10,000 SWU/yr. After some manipulation of Equations (3.1), (3.2), and (3.17), the separative power of the black box production unit can be calculated as

$$
\Delta U=P V\left[N_{P}\right]-F V\left[N_{F}\right]+(F-P) V\left[\frac{N_{F} F-N_{P} P}{F-P}\right]
$$

Solving Equation (7.1) for $P$, assuming a feed rate of $15,000 \mathrm{kgU} / \mathrm{yr}$ of natural uranium 

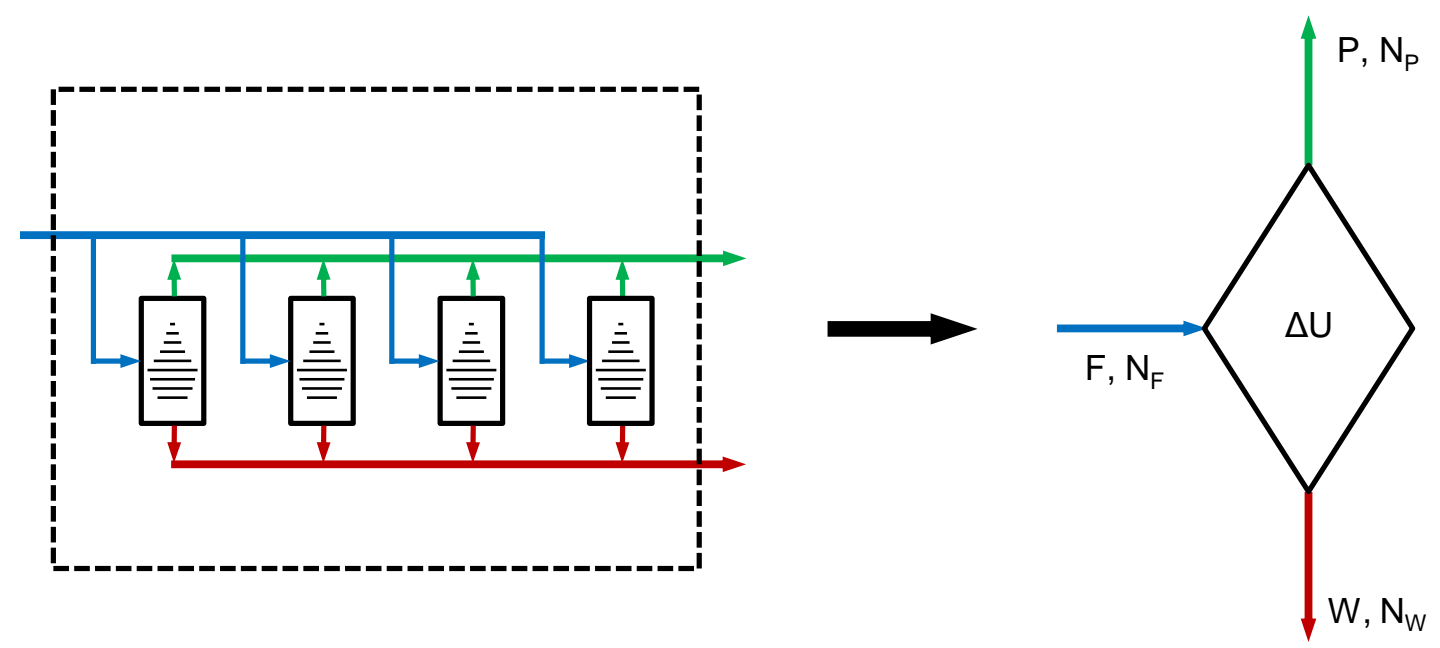

Figure 7.1: Black box treatment of a cascade hall in an ideal separative work analysis. The separative power of all the centrifuges is summed and the separative power equation is used to predict the total product rate. This method neglects mixing inefficiencies that are inherent to real cascade operation.

and a product concentration of $90 \%$, yields a product rate of $56.81 \mathrm{kgU} / \mathrm{yr}$. At this rate, the production unit can produce approximately $1.5 \mathrm{SQ}$ in a year with a breakout time to 1 SQ of 238 days. This ideal analysis ignores many different factors and will be shown to over-predict the production rate by as much as $32 \%$ and under-predict the breakout time by $25 \%$ compared to a fixed plant analysis.

In this chapter, a method for calculating breakout times using a fixed plant analysis is presented and used to analyze the enrichment plant at Natanz. A large portion of this chapter appears in "Fixed Plant Proliferation Analysis of Iran's Natanz Plant" in Proceedings of the INNM 52nd Annual Meeting [104].

\subsection{Multi-Step Enrichment Processes}

Consider a cascade hall with $K$ number of cascades arranged in production units. Assuming that the production units are physically positioned such that they can be joined by piping with relative ease, a multi-step enrichment process can be achieved by grouping the 


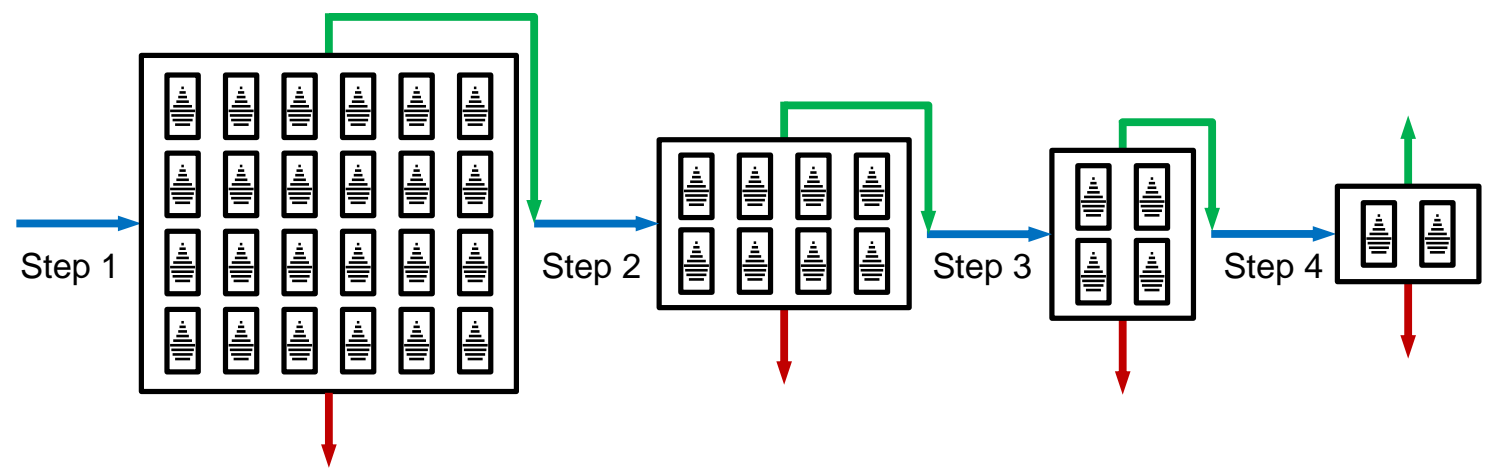

Figure 7.2: Schematic of a multi-step enrichment process via cascade interconnection. Cascades are joined into parallel groupings called steps. Each step is then joined in series much like centrifuges and stages.

production units in series (see Figure 7.2). Additionally, cascades from one production unit can be moved to another so that they are sufficiently distributed. Each grouping will then be referred to as a step, with a maximum of $s=S$ steps. In each step, it is assumed that each cascade operates the same and that the only effect of grouping the cascades is to increase throughput. These specifications are analogous to grouping centrifuges into stages and stages into series.

It is assumed that the multi-step enrichment operation is analogous to a simple cascade, i.e. the product of each step feeds the next step and the tails of each step is not processed as shown in Figure 7.2. This assumption is made because the tails stream concentration of a particular step may not match the concentration of the feed stream of the previous step, introducing mixing. It is assumed that an operator would prefer this method due to its simplicity. To analyze the multi-step enrichment process, a method to calculate the cascade performance in each step is developed. It is assumed that the only knowledge of the cascade is the cascade structure, the type of centrifuge, and target product enrichments for each step. In this case, it is necessary to solve the ideal cascade equations to determine the optimal flow rates in the cascade for each step.

The flow chart for the multi-step enrichment calculation method is shown in Figure 7.3. The calculation begins by defining the inputs: $M_{s}-$ the array containing the number 
of centrifuges in each stage of the cascade for the each step, $F_{1}-$ the cascade feed rate for the first step, $N_{F, 1}-$ the cascade feed concentration for the first step, $C_{S}-$ the number of cascades in each step, and $N_{P T, S}-$ the target product enrichment for each step. Next, the optimal separation parameters for the centrifuge are determined from the performance map and the ideal cascade equations are solved to find the optimal flow rates. The performance map is then called to calculate the stage separation factors and the exact gradient equations are solved to calculate the cascade stage concentrations. If the calculated product enrichment of the cascades matches the target, the product rate is multiplied by the total number of cascades in the step, if not, then the upflow scale factor is adjusted and the previous calculation is repeated until the target is matched. The calculation then proceeds to the next step by feeding the product rate from the previous step to the cascades in the current step. The cascade structure is redefined according to the input specification and the flow and concentration calculation is repeated. The calculation ends when the maximum number of steps is reached.

\subsection{Iran's Natanz Fuel Enrichment Plant}

In August 2002, the National Council of Resistance of Iran disclosed the presence of a secret nuclear facility in Natanz, Iran [105]. Shortly thereafter in December 2002, the Institute for Science and International Security (ISIS) released satellite imagery identifying the facility as a potential gas centrifuge enrichment plant. It wasn't until February 2007, that the first enrichment process at the Fuel Enrichment Plant (FEP) in Natanz began [101].

The enrichment capabilities of the FEP can be determined from IAEA safeguards reports and information delivered by A. Q. Khan, the 'father' of illicit nuclear trade [106]. According to the November 2011 IAEA report on the safeguards status in Iran, the FEP had approximately 8,000 centrifuges installed in 54 cascades. Of these 54, 37 were being fed with $\mathrm{UF}_{6}$ with approximately 6,000 centrifuges [101]. Each cascade is composed of 


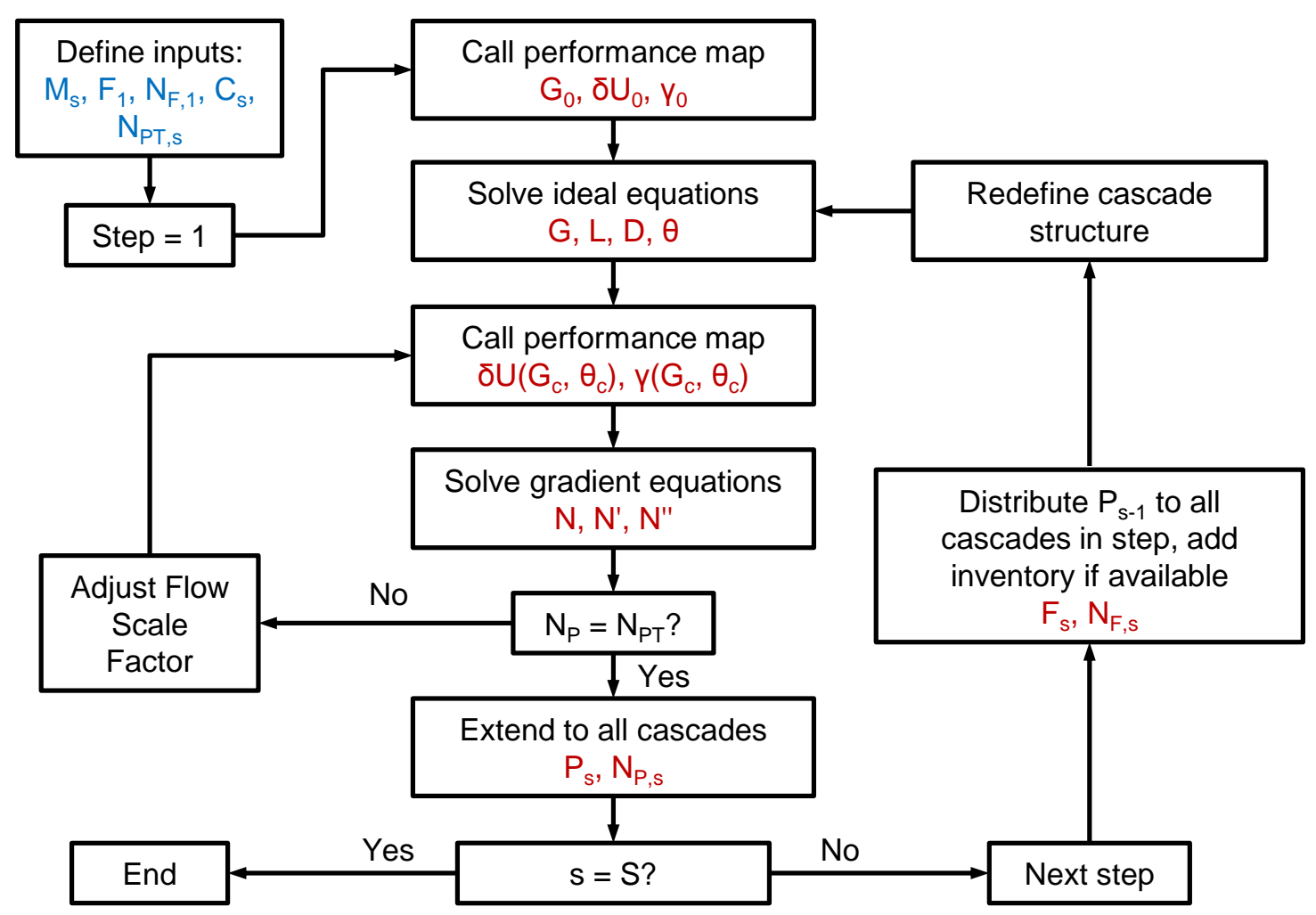

Figure 7.3: Methodology for calculating the performance of a multi-step enrichment process. For a given step and cascade configuration, the exact gradient equations are solved based on target product enrichments for each step. The product rate of each step is used as the feed of the next step. The product rate of the top step is the production rate for the multi-step enrichment process and is used to calculate breakout time.

164 centrifuges for which the structure is given in A. Q. Kahn's confession [106]. This type of cascade was originally designed by URENCO, the enrichment company established by the governments of Germany, the Netherlands, and the United Kingdom. This type of cascade was designed to produce approximately 3.5\% enriched fuel for a power reactor. Khan [106] also described a multi-step enrichment method for which these cascades can be used to produce WGU. The four step process is summarized as:

1. NU is enriched to $3.5 \%{ }^{235} \mathrm{U}$

2. $3.5 \%{ }^{235} \mathrm{U}$ is used as feed to produce $20 \%{ }^{235} \mathrm{U}$

3. $20 \%{ }^{235} \mathrm{U}$ is used as feed to produce $60 \%{ }^{235} \mathrm{U}$

4. $60 \%{ }^{235} \mathrm{U}$ is used as feed to produce $90 \%{ }^{235} \mathrm{U}$ 
In Kahn's plan, for the 15 stage cascade, the number of centrifuges in Steps 3 and 4 were reduced to 114 and 64, respectively.

In an updated safeguards report [107], the IAEA detailed the increase of operational cascades. As of February 2012, 54 cascades were being fed $\mathrm{UF}_{6}$ with roughly 30 cascades modified to contain 174 centrifuges and a total of approximately 9,000 operational centrifuges.

\subsection{IR-1 Centrifuge Performance}

The IR-1 centrifuge is a derivative of the CNOR/SNOR centrifuge, developed by the Netherlands under the URENCO project. The CNOR/SNOR centrifuges were designed to produce roughly 2-3 SWU/yr, however, the performance of the IR-1 centrifuge in cascade has been found to be less than this design point. As determined by Albright and Walrond's analysis of the September 2011 IAEA Iran Safeguards report, the separative power of the IR-1 centrifuge is roughly $0.9 \mathrm{SWU} / \mathrm{yr}$ [100]. As shown in Chapter 5, a performance map of this centrifuge can be generated by specifying a target separative power and separation factor. The separation factor can be estimated by way of ideal cascade analysis. To enrich natural uranium to $3.5 \%{ }^{235} \mathrm{U}$ over 10 enriching stages requires a separation factor of $\gamma=1.3832$. Using these target separation parameters, the semi-empirical method

developed in Chapter 5 is used to calculate the performance of the IR-1 centrifuge over a range of feed rates and cuts. The estimated optimal operating characteristics of the IR-1 centrifuge are shown in Table 7.1. The 2D performance maps for the IR-1 centrifuge are shown in Figure 7.4 with the optimal operating point marked with a white $\mathrm{x}$.

\subsection{Enrichment Plant Capabilities and Breakout Time}

To provide a comprehensive analysis of the enrichment capabilities of the Natanz FEP, the two cascade structures detailed in the previous section are analyzed. The multi-step 
Table 7.1: Estimated operating characteristics for the IR-1 centrifuge

\begin{tabular}{lcc}
\hline Parameter & Unit & Value \\
\hline Number of Stages & - & 24 \\
Stage Enrichment Factor & - & 0.02406 \\
Stage Upflow Rate & $\mathrm{kgU} / \mathrm{yr}$ & 323.3 \\
Optimal Feed Rate & $\mathrm{kgU} / \mathrm{yr}$ & 68.7 \\
Optimal Cut & - & 0.45 \\
Optimal Separative Power & $\mathrm{SWU} / \mathrm{yr}$ & 0.90034 \\
Optimal Separation Factor & - & 1.3832 \\
\hline
\end{tabular}

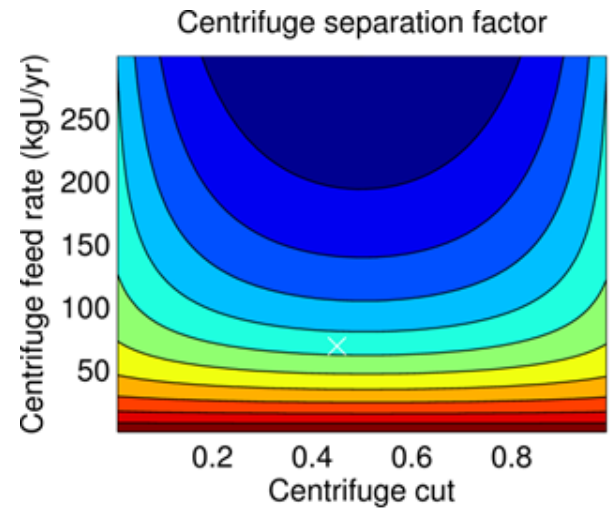

(a) Overall Separation Factor

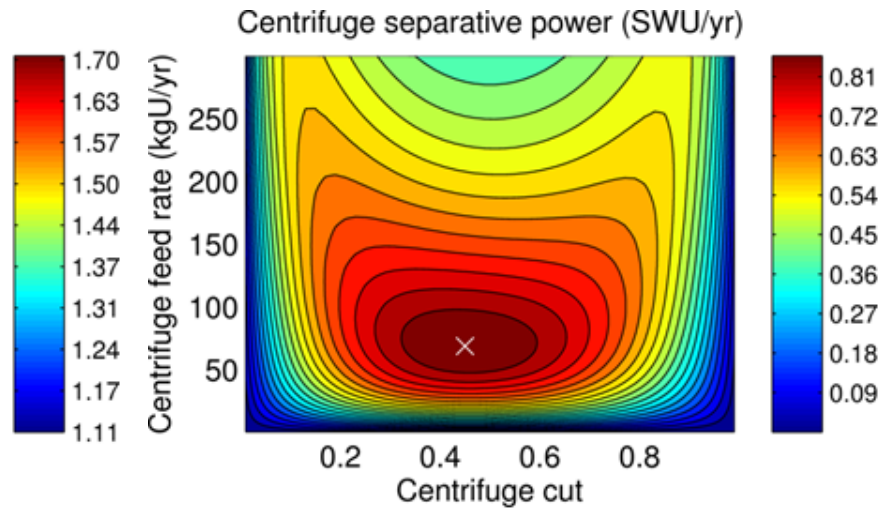

(b) Separative Power

Figure 7.4: $2 \mathrm{D}$ centrifuge performance maps for the IR-1 centrifuge. The performance maps are developed using the semi-empirical method developed in Chapter 6 with target parameters derived from the IAEA safeguards report.

enrichment process for each cascade structure is detailed in Table 7.2, showing the number of cascades in each step and the number of centrifuges in each cascade. For the 15 stage cascade, there are 5,832 centrifuges and 38 cascades distributed amongst the four steps; for the 17 stage cascade, 9,342 centrifuges and 54 cascades. For each of these cascades, two scenarios are considered, with and without inventory. For the with inventory case, the current available inventory $\left(3,042 \mathrm{kgU}\right.$ at $3.5 \%{ }^{235} \mathrm{U}$ and $47.3 \mathrm{kgU}$ at $\left.20 \%{ }^{235} \mathrm{U}\right)$ is distributed to the feed in the appropriate step. The inventory is distributed at a rate of 1 $\mathrm{kgU} / \mathrm{yr}$ per $\mathrm{kgU}$ of material, thereby using the entire inventory in one year. 
Table 7.2: Cascade structure for each enrichment step for the 15 and 17 stage cascades

\begin{tabular}{c|cc|cc}
\hline & \multicolumn{2}{|c|}{ 15 Stages } & \multicolumn{2}{c}{ 17 Stages } \\
\hline Step & Cascades & $\begin{array}{c}\text { Centrifuges } \\
\text { per Cascade }\end{array}$ & Cascades & $\begin{array}{c}\text { Centrifuges } \\
\text { per Cascade }\end{array}$ \\
\hline 1 & 24 & 164 & 30 & 173 \\
2 & 8 & 164 & 12 & 173 \\
3 & 4 & 114 & 8 & 173 \\
4 & 2 & 64 & 4 & 173 \\
\hline
\end{tabular}

In total, four cases are considered:

1. 15 stage cascade without inventory

2. 15 stage cascade with inventory

3. 17 stage cascade without inventory

4. 17 stage cascade with inventory

The multi-step enrichment analysis is performed as described in Section 7.2 for each of the four cases. The first step cascade feed rate is chosen so that the target product concentration of $3.5 \%$ is achieved with a flow scale factor of 1 . The results of the calculations are detailed in Tables 7.3 - 7.6. The table shows the external cascade flow rates and separative power for each cascade and the total for each step. The ideal separative power $\left(\Delta U_{i}\right)$ is the sum of the separative power of all the centrifuge in the cascade. Taking the ratio of the actual separative power to ideal separative power $\left(\Delta U / \Delta U_{i}\right)$ gives the cascade efficiency $(\varepsilon)$. In general, the cascades in Step 1 are the most efficient since they are operating closest to ideal. The addition of inventory in the higher steps serves to increase the efficiency by driving the cascade feed rate towards the ideal rate. In each case, the total Step 4 product rate is used to determine the breakout time as

$$
t_{B}=\frac{25[k g U 235]}{P_{S}\left[\frac{k g U}{y r}\right] \cdot \frac{238[k g U 235]}{352[k g U]}}=\frac{36.975}{P_{4}}[y r]
$$

For the 15 stage cascade, a cascade feed rate of $230.34 \mathrm{kgU} / \mathrm{yr}$ is needed to achieve 
$3.5 \%$ product in the first step. This drives a production rate of $20.63 \mathrm{kgU} / \mathrm{yr}$ for Case 1 and $44.25 \mathrm{kgU} / \mathrm{yr}$ for Case 2. Therefore the time to one SQ of $90 \%$ WGU is 1.79 years (654 days) without inventory available and 0.836 years (305 days) with inventory. In Case 1 , one SQ can be gained every 654 days, for Case 2, the first SQ can be gained in 305 days, the second SQ in another 558 days, and then every subsequent SQ in 654 days. Using an ideal separative work calculation for Case 1 with a total feed rate of 5,528 $\mathrm{kgU} / \mathrm{yr}$ and total separative power of 5,250.8 SWU/yr yields a production rate of $26.49 \mathrm{kgU} / \mathrm{yr}$ and a breakout time of 1.39 years (510 days). The ideal calculation over-predicts the production rate by $28 \%$ and under-predicts the breakout time by $22 \%$.

For the 17 stage cascade, a cascade feed rate of $382.39 \mathrm{kgU} / \mathrm{yr}$ is needed to achieve $3.5 \%$ product in the first step. This drives a production rate of $35.02 \mathrm{kgU} / \mathrm{yr}$ for Case 3 and $80.77 \mathrm{kgU} / \mathrm{yr}$ for Case 4. Therefore the time to one SQ of $90 \% \mathrm{WGU}$ is 1.06 years (385 days) without inventory available and 0.458 years (167 days) with inventory. In Case 3 , one SQ can be gained every 385 days, for Case 2, the first SQ can be gained in 167 days, the second SQ in another 167 days, the third SQ in another 346 days, and every subsequent SQ in 385 days. The ideal calculation for Case 3 with a total feed rate of 11,471 kgU/yr and total separative power of $8410.97 \mathrm{SWU} / \mathrm{yr}$ gives a production rate of $46.44 \mathrm{kgU} / \mathrm{yr}$ and a breakout time of 0.796 years (291 days). This calculation over-predicts the production rate by $32 \%$ and under-predicts the breakout time by $25 \%$.

\subsection{Summary}

In this chapter, a method to analyze the capabilities of an enrichment plant operating in a multi-step WGU process was developed. The method was used to predict the production rate and breakout time of the Fuel Enrichment Plant in Natanz, Iran. Two different cascade structures corresponding to those reported in IAEA safeguards reports are studied. For both cascade, two cases are presented, one where existing inventory of $3.5 \%$ and $20 \%$ material 
Table 7.3: Summary of cascade results for Case 1: 15 Stage Cascade, No Inventory

\begin{tabular}{l|cc|cc|cc|cc}
\hline & \multicolumn{2}{|c|}{ Step 1 } & \multicolumn{2}{c|}{ Step 2 } & \multicolumn{2}{c|}{ Step 3 } & \multicolumn{2}{c}{ Step 4 } \\
Parameter & Cascade & Total & Cascade & Total & Cascade & Total & Cascade & Total \\
\hline$F(\mathrm{kgU} / \mathrm{yr})$ & 230.34 & 5528.0 & 94.57 & 756.55 & 26.74 & 106.94 & 16.43 & 32.87 \\
$P(\mathrm{kgU} / \mathrm{yr})$ & 31.52 & 756.55 & 13.37 & 106.94 & 8.22 & 32.87 & 10.31 & 20.63 \\
$W$ (kgU/yr) & 198.81 & 4771.5 & 81.20 & 649.62 & 18.52 & 74.09 & 6.12 & 12.23 \\
$N_{W}$ (at. frac.) & 0.0071 & 0.0071 & 0.0350 & 0.0350 & 0.2000 & 0.2000 & 0.6000 & 0.6000 \\
$N_{P}$ (at. frac.) & 0.0350 & 0.0350 & 0.2000 & 0.2000 & 0.6000 & 0.6000 & 0.9000 & 0.9000 \\
$N_{W}$ (at. frac.) & 0.0027 & 0.0027 & 0.0078 & 0.0078 & 0.0226 & 0.0226 & 0.0941 & 0.0941 \\
$\Delta U$ (SWU/yr) & 145.67 & 3496.2 & 106.35 & 850.82 & 45.07 & 180.29 & 28.04 & 56.08 \\
$\Delta U_{i}(\mathrm{SWU} / \mathrm{yr})$ & 146.76 & 3522.1 & 146.76 & 1174.0 & 102.64 & 410.56 & 57.62 & 115.24 \\
$\varepsilon(\%)$ & 99.26 & 99.26 & 72.47 & 72.47 & 43.91 & 43.91 & 48.66 & 48.66 \\
$\zeta$ & 1.0000 & 1.0000 & 1.0745 & 1.0745 & 0.7837 & 0.7837 & 0.7270 & 0.7270 \\
\hline
\end{tabular}

Table 7.4: Summary of cascade results for Case 2: 15 Stage Cascade, With Inventory

\begin{tabular}{l|cc|cc|cc|cc}
\hline & \multicolumn{2}{|c|}{ Step 1 } & \multicolumn{2}{c|}{ Step 2 } & \multicolumn{2}{c|}{ Step 3 } & \multicolumn{2}{c}{ Step 4 } \\
Parameter & Cascade & Total & Cascade & Total & Cascade & Total & Cascade & Total \\
\hline$F(\mathrm{kgU} / \mathrm{yr})$ & 230.34 & 5528.0 & 474.82 & 3798.6 & 71.76 & 287.04 & 39.29 & 78.59 \\
$P(\mathrm{kgU} / \mathrm{yr})$ & 31.52 & 756.55 & 29.97 & 239.74 & 19.65 & 78.59 & 22.12 & 44.25 \\
$W$ (kgU/yr) & 198.81 & 4771.5 & 444.85 & 3558.8 & 52.11 & 208.45 & 17.17 & 34.33 \\
$N_{W}$ (at. frac.) & 0.0071 & 0.0071 & 0.0350 & 0.0350 & 0.1975 & 0.1975 & 0.6000 & 0.6000 \\
$N_{P}$ (at. frac.) & 0.0350 & 0.0350 & 0.1975 & 0.1975 & 0.6000 & 0.6000 & 0.9000 & 0.9000 \\
$N_{W}$ (at. frac.) & 0.0027 & 0.0027 & 0.0241 & 0.0241 & 0.0458 & 0.0458 & 0.2134 & 0.2134 \\
$\Delta U$ (SWU/yr) & 145.67 & 3496.2 & 128.89 & 1031.2 & 84.53 & 338.13 & 48.54 & 97.09 \\
$\Delta U_{i}($ SWU/yr) & 146.76 & 3522.1 & 146.76 & 1174.0 & 102.64 & 410.56 & 57.62 & 115.24 \\
$\varepsilon(\%)$ & 99.26 & 99.26 & 87.82 & 87.82 & 82.36 & 82.36 & 84.24 & 84.24 \\
$\zeta$ & 1.0000 & 1.0000 & 2.4066 & 2.4066 & 0.8746 & 0.8746 & 0.8105 & 0.8105 \\
\hline
\end{tabular}

is used and one where it isn't, resulting in a total of four scenarios.

For the 15 stage cascade, the time to one SQ of WGU varies between 305-654 days (1022 months), depending on if inventory is used. For the 17 stage cascade, the breakout time is between 167-385 days (5.5-13 months). It is also shown that using an ideal separative work calculation under-predicts the breakout time by $22 \%$ for the 15 stage cascade and $25 \%$ for the 17 stage cascade.

It is clear that if a country does decide to pursue weapon grade enriched material for a nuclear weapon, the time to achieve enough material is highly dependent on the cascade structure and availability of stockpiles of LEU. Ideal separative work calculations can provide a minimum breakout time, but it is important to note that these estimates may 
Table 7.5: Summary of cascade results for Case 3: 17 Stage Cascade, No Inventory

\begin{tabular}{l|cc|cc|cc|cc}
\hline & \multicolumn{2}{|c|}{ Step 1 } & \multicolumn{2}{c|}{ Step 2 } & \multicolumn{2}{c|}{ Step 3 } & \multicolumn{2}{c}{ Step 4 } \\
Parameter & Cascade & Total & Cascade & Total & Cascade & Total & Cascade & Total \\
\hline$F(\mathrm{kgU} / \mathrm{yr})$ & 382.39 & 11471 & 93.79 & 1125.5 & 21.14 & 169.11 & 13.50 & 53.99 \\
$P(\mathrm{kgU} / \mathrm{yr})$ & 37.52 & 1125.5 & 14.09 & 169.11 & 6.75 & 53.99 & 8.76 & 35.02 \\
$W$ (kgU/yr) & 344.87 & 10346 & 79.70 & 956.37 & 14.39 & 115.13 & 4.74 & 18.98 \\
$N_{W}$ (at. frac.) & 0.0071 & 0.0071 & 0.0350 & 0.0350 & 0.2000 & 0.2000 & 0.6000 & 0.6000 \\
$N_{P}$ (at. frac.) & 0.0350 & 0.0350 & 0.2000 & 0.2000 & 0.6000 & 0.6000 & 0.9044 & 0.9044 \\
$N_{W}$ (at. frac.) & 0.0041 & 0.0041 & 0.0058 & 0.0058 & 0.0124 & 0.0124 & 0.0381 & 0.0381 \\
$\Delta U$ (SWU/yr) & 134.77 & 4043.02 & 127.27 & 1527.2 & 44.39 & 355.12 & 28.98 & 115.91 \\
$\Delta U_{i}$ (SWU/yr) & 155.76 & 4672.76 & 155.76 & 1869.1 & 155.76 & 1246.07 & 155.76 & 623.04 \\
$\varepsilon(\%)$ & 86.52 & 86.52 & 81.71 & 81.71 & 28.49 & 28.49 & 18.61 & 18.61 \\
$\zeta$ & 1.0000 & 1.0000 & 0.7575 & 0.7575 & 0.6475 & 0.6475 & 0.6660 & 0.6660 \\
\hline
\end{tabular}

Table 7.6: Summary of cascade results for Case 4: 17 Stage Cascade, With Inventory

\begin{tabular}{l|cc|cc|cc|cc}
\hline & \multicolumn{2}{|c|}{ Step 1 } & \multicolumn{2}{c|}{ Step 2 } & \multicolumn{2}{c|}{ Step 3 } & \multicolumn{2}{c}{ Step 4 } \\
Parameter & Cascade & Total & Cascade & Total & Cascade & Total & Cascade & Total \\
\hline$F(\mathrm{kgU} / \mathrm{yr})$ & 382.39 & 11471 & 347.29 & 4167.5 & 51.22 & 409.80 & 31.36 & 125.42 \\
$P(\mathrm{kgU} / \mathrm{yr})$ & 37.52 & 1125.5 & 30.21 & 362.50 & 15.68 & 125.42 & 20.19 & 80.77 \\
$W$ (kgU/yr) & 344.87 & 10346 & 317.08 & 3804.9 & 35.54 & 284.34 & 11.17 & 44.67 \\
$N_{W}$ (at. frac.) & 0.0071 & 0.0071 & 0.0350 & 0.0350 & 0.1975 & 0.1975 & 0.6000 & 0.6000 \\
$N_{P}$ (at. frac.) & 0.0350 & 0.0350 & 0.1975 & 0.1975 & 0.6000 & 0.6000 & 0.9000 & 0.9000 \\
$N_{W}$ (at. frac.) & 0.0041 & 0.0041 & 0.0195 & 0.0195 & 0.0200 & 0.0200 & 0.0575 & 0.0575 \\
$\Delta U$ (SWU/yr) & 134.77 & 4043.0 & 147.79 & 1773.5 & 90.71 & 725.71 & 60.60 & 242.40 \\
$\Delta U_{i}$ (SWU/yr) & 155.76 & 4672.8 & 155.76 & 1869.1 & 155.76 & 1246.1 & 155.76 & 623.04 \\
$\varepsilon(\%)$ & 86.52 & 86.52 & 94.88 & 94.88 & 57.78 & 57.78 & 38.91 & 38.91 \\
$\zeta$ & 1.0000 & 1.0000 & 1.3085 & 1.3085 & 0.6708 & 0.6708 & 0.6708 & 0.6708 \\
\hline
\end{tabular}

be at $25 \%$ off of a more realistic analysis. In addition to enrichment time, one should also consider the time it takes to restructure the cascade hall for the multi-step enrichment process and material conversion time. 


\section{Chapter 8}

\section{Transient Modeling of Gas Centrifuge Cascades}

\subsection{Overview}

As discussed in Chapter 1, in light of the development of new safeguards technologies to monitor and assess the operation of a GCEP, it is important to gain an understanding of the dynamics of a particular cascade. In cases where new unattended monitoring systems are not installed or have failed, it is important to quantify the time scales associated with different proliferation scenarios in order to determine how long detectable phenomena will last in the cascade. In the case where on-line enrichment monitors and cylinder weighing are installed, it can be helpful to determine what signatures of misuse look like and how they differ from accidental, non-malicious, off-normal operation such as centrifuge failure.

In this chapter, a transient fluid dynamics and isotope separation model are developed and implemented in a software package called TransCasc. The code runs on the Simscape and Simulink platform associated with MATLAB. The flow model is verified by comparison with an existing model in the literature. An illustrative study of proliferation time-frames and signatures of misuse is presented with several generic cascades operating in various off-normal operation modes.

A large portion of this chapter is derived from "Transient Fluid Dynamics Modeling of Gas Centrifuge Enrichment Plants" in Proceedings of the 52nd Annual Meeting of the 
INMM [108], "Transient Operation of a Gas Centrifuge Cascade to Determine Proliferation Time Frames" in Proceedings of the 12th Workshop on Separation Phenomena in Liquids and Gases [109], and "Transient Isotope Separation Modeling of Gas Centrifuge Enrichment Plants" in Proceedings of the 53rd Annual Meeting of the INMM [110].

\subsection{Transient Flow Modeling}

Proliferation represents an off-normal operation of a gas centrifuge cascade. This offnormal operation requires an alteration of the material streams in the cascade and thus a change in the enrichment for most cases. In order to fully study the dynamics of these varying operating states, it is necessary to both develop a transient fluid dynamics model and a transient isotope separation model of a cascade. These two models can then be coupled, providing the ability to simulate dynamic changes to the cascade operation and allowing the quantification of proliferation time-frames and the study of signatures of misuse.

\subsubsection{Simscape}

To develop a fluid dynamics model of a generic gas centrifuge cascade, Simscape (The Mathworks, Natick, MA) is employed. Simscape is the physical modeling tool associated with Simulink and MATLAB. The advantage of Simscape lies in the "Physical Network" approach which allows the user to create mathematical models of physical systems by connecting components much like a physical system would be created. In Simscape, several domains exist to model different types of components such as Electrical, Hydraulic, Mechanical, Pneumatic, and Thermal. Since a gas centrifuge cascade is a complex pipe network with compressible flow, the Pneumatic domain is most appropriate. 
Pneumatic elements model the transport of a gas under the following assumptions:

1. The fluid is an ideal gas

2. The specific heats at constant pressure and constant volume are constant

3. Gravitational effects are negligible

The first assumption is suitable as $\mathrm{UF}_{6}$ behaves fairly close to an ideal gas [111]. The second assumption is valid in the isothermal case which is appropriate because in a real cascade, the temperature is typically held constant or close to constant. The third assumption is appropriate because we wish to neglect the intricate details of the piping network and instead focus on the holdup (inventory) of the system. We will assume that all minor head loss effects (due to flow through valves, pipe bends, fittings, gravity, etc.) can be tuned by a pipe aggregate length factor.

For these assumptions, and in general, the continuity equation of each component associated with a volume is

$$
V \frac{\partial \rho}{\partial t}=\sum_{i}\left(\dot{m}_{i}\right)-\sum_{o}\left(\dot{m}_{o}\right)
$$

where $\rho$ is the gas density, $V$ is the volume of the component, $\dot{m}$ is the mass flow rate, and the subscripts $i$ and $o$ refer to the flow in and out of the control volume respectively [112]. The general energy equation associated with each component is

$$
\frac{d E}{d t}=Q-W+\sum_{i}\left[\dot{m}_{i}\left(h_{i}+\frac{1}{2} u_{i}^{2}\right)\right]-\sum_{o}\left[\dot{m}_{o}\left(h_{o}+\frac{1}{2} u_{o}^{2}\right)\right]
$$

where $E$ is the total energy in the control volume, $Q$ is the heat added per unit time to the gas, $W$ is the work performed by the gas per unit time, $h$ is the enthalpy of the gas, and $u$ is the gas velocity.

To create a model of a gas centrifuge cascade, three important pneumatic elements are used: the pneumatic resistive tube, the constant volume pneumatic chamber, and the controlled pneumatic flow rate source. 


\section{Pneumatic Resistive Tube}

The pneumatic resistive tube models the pressure drop in a short section of circular pipe due to viscous resistance. For a circular cross-sectional, adiabatic pipe, the pressure loss across the length of the pipe is

$$
p_{i}-p_{o}= \begin{cases}\frac{R_{g} T}{p_{i}} \cdot \frac{32 \mu L_{p}}{A_{p} D_{p}^{2}} & : \operatorname{Re}<\operatorname{Re}_{\mathrm{lam}} \\ f \cdot \frac{R_{g} T}{p_{i}} \cdot \frac{L_{p}}{D_{p}} \cdot \frac{\dot{m}^{2}}{2 A_{p}^{2}} & : \operatorname{Re}>\mathrm{Re}_{\mathrm{turb}}\end{cases}
$$

where $\mu$ is the gas viscosity, $L$ is the length of the pipe, $D_{p}$ is the diameter of the pipe, $A_{p}$ is the cross-sectional area of the pipe, $f$ is the friction factor determined by

$$
f=\left\{-1.8 \log \left[\frac{6.9}{\operatorname{Re}}+\left(\frac{e}{3.7 D_{p}}\right)^{1.11}\right]\right\}^{-2}
$$

where $e$ is the surface roughness of the pipe and Re is the Reynolds number defined as

$$
\operatorname{Re}=\frac{4 \dot{m}}{\pi D_{p} \mu}
$$

with $\operatorname{Re}_{\text {lam }}=2,000$ and $\operatorname{Re}_{\text {turb }}=4,000$. For Reynolds numbers between these values (transition flow), the pressure drop is linearly interpolated between the two pressure drop models.

\section{Constant Volume Pneumatic Chamber}

The constant volume pneumatic chamber block models a constant volume of gas based on the ideal gas law and constant specific heats. For the block, the continuity equation is

$$
\dot{m}=\frac{V}{R_{g} T}\left[\frac{d p}{d t}-\frac{p}{T} \frac{d T}{d t}\right]
$$

and the energy equation is 


$$
q=\frac{c_{v} V}{R_{g}} \cdot \frac{d p}{d t}-q_{w}
$$

where $q_{w}$ is the heat transfer through the chamber walls, which is assumed to be zero in this case.

\section{Controlled Pneumatic Flow Rate Source}

The controlled pneumatic flow rate source models an ideal compressor that maintains a specified mass flow rate. Three ports determine the flow through the block: input, output, and signal. The signal port is used to specify the mass flow rate across the block.

\subsubsection{Modeling a Gas Centrifuge Cascade}

As detailed in Chapter 4, a gas centrifuge cascade is complex pipe network with various components including centrifuges, valves, and sensors. It is the goal of this research to develop a model of the cascade that incorporates only the most prevalent components of the cascade based on information that an inspector may gain from a visual inspection or have available in a DIQ. The model is developed assuming that each centrifuge in a given stage operates the same, and thus, the cascade is studied at the stage level. This is appropriate for studying off-normal scenarios where the piping in each stage is fixed and flows can be altered by tuning valves and attaching portable feed and withdrawal systems to access ports. The above components are used to develop the model of the gas centrifuge cascade.

\section{Transient Gas Flow in a Pipe}

To model the transient flow of gas in a pipe, the pneumatic resistive tube block is connected to the constant volume pneumatic chamber. This lumped parameter method can be achieved with one or several segments. The ideal number of segments should be examined. The equivalent circuit of the pipe segments and chambers accounts for the pressure drop due 


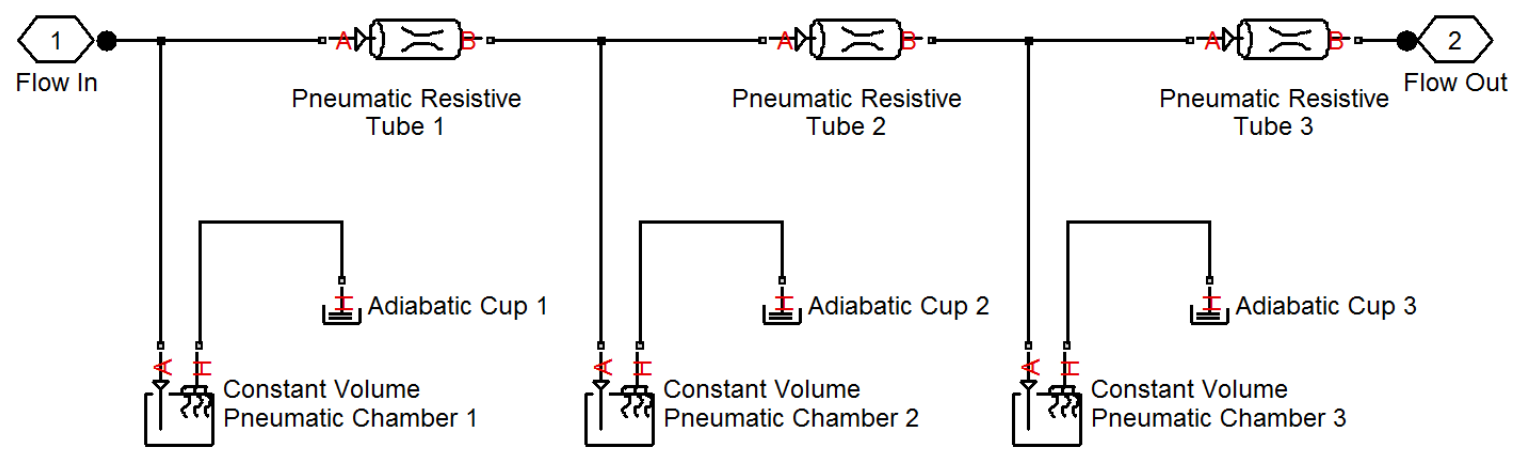

Figure 8.1: Diagram of the lumped parameter pipe model developed in Simscape. The pipe model accounts for fluid compressibility and viscous pressure loss.

to viscous drag and the compressibility of the gas. Heating due to friction is neglected as the flow through the cascade is relatively slow (on the order of $0.01 \mathrm{~kg} / \mathrm{s}$ in the maximum case). In each segment, the pipe length and volume is distributed evenly amongst each segment. The length of the pipe is assumed to be proportional to the number of centrifuges in the stage as $L_{\text {pipe }}=F_{p} \cdot 2 a \cdot N_{c}$ and the equivalent length of pipe due to the presence of local resistances is $L_{\mathrm{eq}}=\left(F_{p}+F_{a}\right) \cdot 2 a \cdot N_{c}$. For each tube segment, the tube length is determined by $L_{p}=L_{\mathrm{eq}} / N_{s}$ and for each pneumatic chamber segment the chamber volume is determined by $V=A_{p} \cdot L_{\text {pipe }} / N_{s}$, where $N_{s}$ is the number of segments. Here, $F_{p}$ is denotes the pipe length factor and $F_{a}$ is the pipe aggregate length factor. Together with the pipe diameter, these two parameters are used to characterize the amount of holdup in the pipes and the pressure drop across the cascade. Figure 8.1 shows a diagram of the lumped parameter pipe model with three segments.

\section{Stage Model}

In each cascade stage, flow enters the feed header and is distributed to each centrifuge in that stage. The material is then enriched/depleted and leaves though product and tails headers. In a real cascade, these flows are controlled through pressure regulation and valve controls to maintain a stage cut. To model the stage (see Figure 8.2), a feed pipe is connected to a constant volume pneumatic chamber (blue) representing the centrifuge. As 


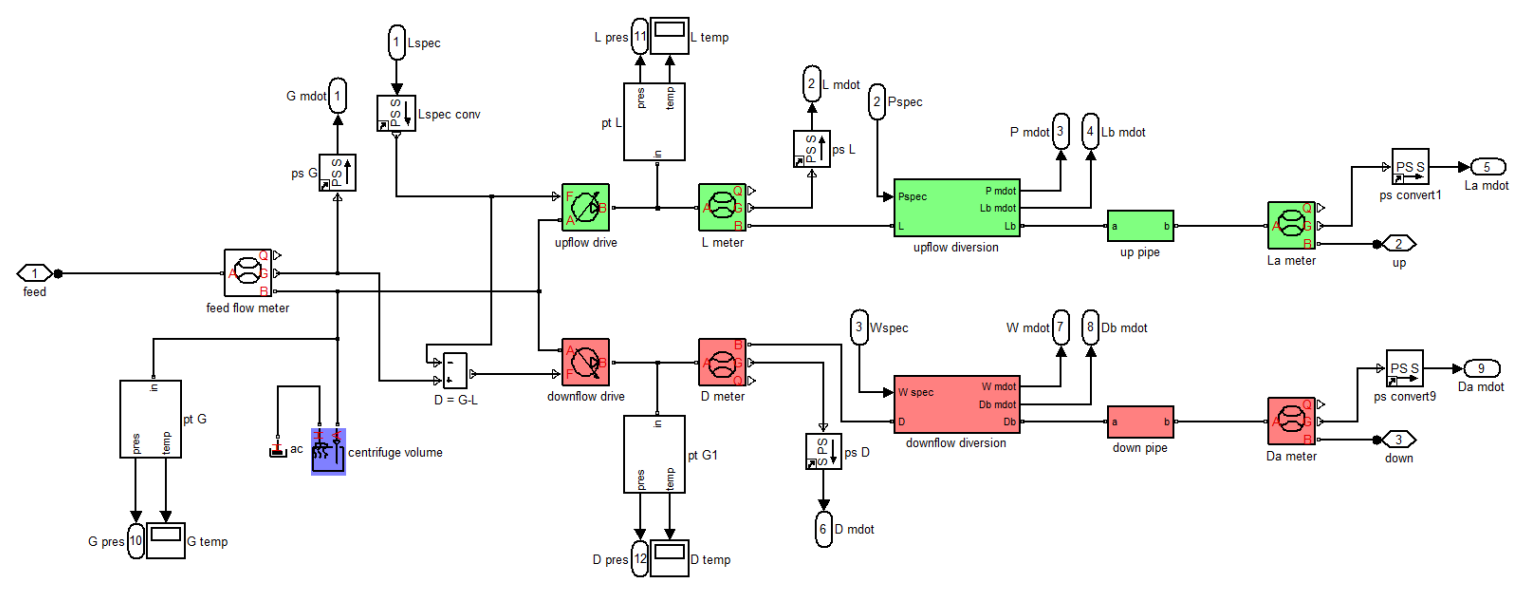

Figure 8.2: Diagram of a cascade stage model with specified stage upflow rate. The centrifuge is modeled as a volume connected to two ideal compressors. The upflow and downflow through the centrifuge is determined by a user-specified upflow rate. Downstream of the centrifuge is a diversion pathway and a header pipe that leads to the next stage.

a simplification, the hydrodynamics of the centrifuge are not considered and the centrifuge it treated as two compressors with storage. The centrifuge separates the flow into two paths, the upflow (green) and downflow (red), with flow rate determined by two controlled pneumatic flow rate sources. A diversion pathway is connected downstream of the the controlled flow rate sources to allow for addition and withdrawal of material. Downstream of the diversion pathway is the header pipe which exits to the feed point of the next stage.

For this research, two flow control models will be incorporated: specified stage cut and specified stage upflow rate. The two flow control models introduce a minor difference in the way the upflow and downflow rates are controlled, allowing the user to simulate a broader range of off-normal operations.

The volume for the pneumatic chamber representing the centrifuge is determined by considering the gas inventory in the centrifuge. The mass inventory or holdup inside the gas centrifuge is calculated as

$$
I=\int_{0}^{a} 2 \pi r H \rho(r) d r
$$

Recalling the base state pressure distribution in the centrifuge and the equation of state, 
Equation (8.8) can be solved to find

$$
I=\frac{p_{w}}{R_{g} T_{0}} \cdot\left[\frac{\pi H a^{2}\left(1-e^{-A^{2}}\right)}{A^{2}}\right]=\rho_{w} \cdot V_{\mathrm{eff}}
$$

where the first term is the density of the gas at the wall and the term in the brackets represents an effective volume. This effective volume is used to represent the volume of the centrifuge in the pneumatic chamber block of the stage.

Each block that has an associated volume requires an initial pressure and temperature. In this model, the centrifuge wall pressure and average temperature are used. At the feed point of the cascade, a constant volume pneumatic chamber joined with a controlled flow rate source is used to pump feed material into the cascade. At the product and tails withdrawal points of the cascade, material from the cascade flows into a constant volume pneumatic chamber. These are specified as chambers with large volumes to apply a constant pressure boundary condition.

There are two good experimental studies that characterize the transport properties of $\mathrm{UF}_{6}$, the work of DeWitt [111] and Zarkova et. al. [113]. Most of the transport properties are found to be a function of temperature alone. The viscosity, specific heat at constant pressure, and ratio of specific heats are, respectively

$$
\begin{gathered}
\mu(T)=\left[(3.907)+(0.03830) \cdot T+\left(2.783 \cdot 10^{-5}\right) \cdot T^{2}\right. \\
\left.+\left(-2.099 \cdot 10^{-8}\right) \cdot T^{3}\right]\left(1 \cdot 10^{6}\right) \cdot[\mathrm{Pa} \cdot \mathrm{s}] \\
c_{p}(T)=\left[32.43+\left(7.936 \cdot 10^{-3}\right) \cdot T+\left(-32.068 \cdot 10^{4}\right) \cdot T^{-2}\right] \cdot \frac{1}{M} \cdot 4.184 \cdot\left[\frac{J}{\mathrm{~kg} \cdot K}\right] \\
\frac{c_{p}}{c_{v}} \approx 1.062
\end{gathered}
$$


where $T$ is the temperature in Kelvin and $M$ is the molar mass of the gas in $\mathrm{kg} / \mathrm{mol}$.

\subsection{Transient Isotope Separation Model}

In Chapters 2 and 3, the theory of isotope separation was developed for the centrifuge and cascade assuming steady-state operation. In this work, it is assumed that the transient isotope separation phenomena in the centrifuge does not significantly contribute to the overall transient in the cascade. This assumption is made by considering the effective volume of the centrifuge in a stage and the volume of the pipes. A given particle of fluid will travel through only one centrifuge in a stage, but will travel through an entire header pipe. For most stages the inventory of the header pipes will be much greater than the centrifuge inventory. This assumption has also been made by other researchers [93]. The transient isotope separation model developed for this research will follow closely the work of Zeng and Ying [114-116].

Consider a general stage $n$ with feed stream and upflow and downflow stream (see Figure 8.3). Across the stage, the total material balance and isotopic material balances for the $i^{t h}$ isotope are respectively

$$
\begin{gathered}
\frac{\partial I_{n}}{\partial t}=G_{n}-L_{n}-D_{n} \\
\frac{\partial I_{n} \bar{N}_{n, i}}{\partial t}=G_{n} N_{n, i}-L_{n} N_{n, i}^{\prime}-D_{n} N_{n, i}^{\prime \prime}
\end{gathered}
$$

where $I_{n}$ is the centrifuge inventory, $\bar{N}_{n, i}$ is the average concentration across the stage of the $i^{t h}$ isotope, $G_{n}$ is the stage feed rate, $N_{n, i}$ is the concentration of the $i^{t h}$ isotope is the feed stream, $L_{n}$ is the stage upflow rate, $N_{n, i}^{\prime}$ is the concentration of the $i^{t h}$ isotope in the upflow stream, $D_{n}$ is the stage downflow rate, and $N_{n, i}^{\prime \prime}$ is the concentration of the $i^{\text {th }}$ isotope in the 


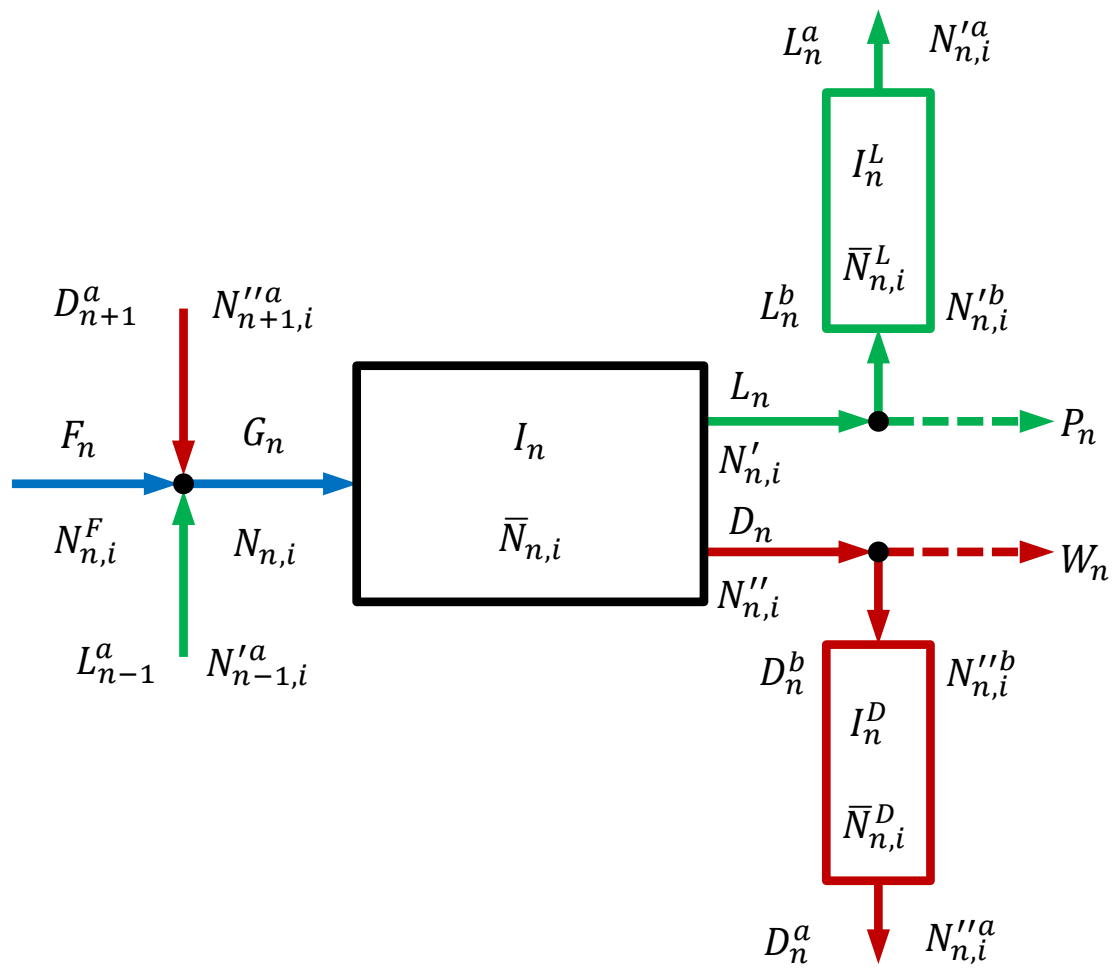

Figure 8.3: Flow diagram of a general cascade stage. Feed material enters the stage through a feed point and into an associated stage holdup. The enriched upflow stream travels through a withdrawal point, into a header pipe, and to the next stage. Similarly, the downflow stream is transported to the previous stage.

downflow stream.

At the feed point, with negligible volume, the overall and isotopic material balances are

$$
\begin{gathered}
G_{n}=F_{n}+L_{n-1}^{a}+D_{n+1}^{a} \\
G_{n} N_{n, i}=F_{n} N_{n, i}^{F}+L_{n-1}^{a} N_{n-1, i}^{\prime a}+D_{n+1}^{a} N_{n+1, i}^{\prime \prime a}
\end{gathered}
$$

where $F_{n}$ is additional material added to the stage, $N_{n, i}^{F}$ is the concentration of the additional material, and the superscript $a$ denotes values of flow exiting the header pipe. At the point just before entering the header piping, the overall material balances are 


$$
\begin{gathered}
L_{n}=L_{n}^{b}+P_{n} \\
D_{n}=D_{n}^{b}+W_{n}
\end{gathered}
$$

where $P_{n}$ is material flow removed from (or added to) the upflow stream, $W_{n}$ is material flow removed from (or added to) the downflow stream, and the superscript $b$ denotes values before entering the header pipe. It is assumed that material withdrawn or added is at the same concentration of that leaving the stage. This gives: $N_{n, i}^{\prime b}=N_{n, i}^{\prime}$ and $N_{n, i}^{\prime \prime b}=N_{n, i}^{\prime \prime}$.

Across the upflow header pipe and downflow header pipe, the material and isotopic material balances are

$$
\begin{gathered}
\frac{\partial I_{n}^{L}}{\partial t}=L_{n}^{b}-L_{n}^{a} \\
\frac{\partial I_{n}^{L} \bar{N}_{n, i}^{L}}{\partial t}=L_{n}^{b} N_{n, i}^{\prime b}-L_{n}^{a} N_{n, i}^{\prime a} \\
\frac{\partial I_{n}^{D}}{\partial t}=D_{n}^{b}-D_{n}^{a} \\
\frac{\partial I_{n}^{D} \bar{N}_{n, i}^{D}}{\partial t}=D_{n}^{b} N_{n, i}^{\prime \prime b}-D_{n}^{a} N_{n, i}^{\prime \prime a}
\end{gathered}
$$

where the superscript $L$ denotes values associated with the upflow header pipe and the superscript $D$ denotes values associated with the downflow header pipe.

Using the product rule and substituting Equation (8.13), (8.15) and (8.16) into Equation (8.14) yields 


$$
\begin{array}{r}
\frac{\partial \bar{N}_{n, i}}{\partial t}=\frac{1}{I_{n}} \cdot\left[-\bar{N}_{n, i}\left(F_{n}+L_{n-1}^{a}+D_{n+1}^{a}-L_{n}-D_{n}\right)\right. \\
\left.+F_{n} N_{n, i}^{F}+L_{n-1}^{a} N_{n-1, i}^{\prime a}+D_{n+1}^{a} N_{n+1, i}^{\prime \prime a}-L_{n} N_{n, i}^{\prime}-D_{n} N_{n, i}^{\prime \prime}\right]
\end{array}
$$

Similarly for Equations (8.20) and (8.22)

$$
\begin{aligned}
& \frac{\bar{N}_{n, i}^{L}}{\partial t}=\frac{1}{I_{n}^{L}} \cdot\left[-\bar{N}_{n, i}^{L}\left(\left(L_{n}-P_{n}\right)-L_{n}^{a}\right)+\left(L_{n}-P_{n}\right) N_{n, i}^{\prime}-L_{n}^{a} N_{n, i}^{\prime a}\right] \\
& \frac{\bar{N}_{n, i}^{D}}{\partial t}=\frac{1}{I_{n}^{D}} \cdot\left[-\bar{N}_{n, i}^{D}\left(\left(D_{n}-W_{n}\right)-D_{n}^{a}\right)+\left(D_{n}-W_{n}\right) N_{n, i}^{\prime \prime}-D_{n}^{a} N_{n, i}^{\prime \prime a}\right]
\end{aligned}
$$

In order to proceed with the solution of Equations (8.23)-(8.25), some extra relationships between the cascade concentrations must be made. One such relationship is the stage separation factor, which for the gas centrifuge is defined as

$$
\gamma_{i, j}=\frac{N_{n, i}^{\prime}}{N_{n, i}^{\prime \prime}} \cdot \frac{N_{n, j}^{\prime \prime}}{N_{n, j}^{\prime}}=\gamma_{u, n}^{M_{j}-M_{i}}
$$

where $\gamma_{u, n}$ is the unity overall separation factor, and $M_{i}$ and $M_{j}$ are the mass numbers of the $i^{t h}$ and $j^{t h}$ components of the mixture. Because it is assumed that the centrifuge transient does not appreciably contribute to the overall cascade transient, the separation factor is indirectly a function of time through the centrifuge feed rate and cut, i.e. $\gamma_{u}=f(G(t), \theta(t))$. Additionally, the summation of all components in a mixture must equal one, or

$$
\sum_{i=1}^{J} N_{n, i}^{\prime}=\sum_{i=1}^{J} N_{n, i}^{\prime \prime}=\sum_{i=1}^{J} N_{n, i}^{\prime a}=\sum_{i=1}^{J} N_{n, i}^{\prime \prime a}=1
$$

The average concentration in the stage and pipes can be determined by taking a massweighted average over the flow domain of the centrifuge and pipes respectively. Because 
the internal hydrodynamics in the centrifuge are not considered in the fluid dynamics model, following Zeng and Ying [116], the average stage concentration is approximated by taking a flow rate weighted average of the upflow and downflow concentrations as

$$
\bar{N}_{n, i}=\frac{\left(L_{n}+P_{n}\right) N_{n, i}^{\prime}+\left(D_{n}+W_{n}\right) N_{n, i}^{\prime \prime}}{L_{n}+P_{n}+D_{n}+W_{n}}
$$

Instead of assuming $\bar{N}_{n, i}^{L}=N_{n, i}^{\prime a}$ and $\bar{N}_{n, i}^{D}=N_{n, i}^{\prime \prime a}$ as Zeng and Ying did, a flow rate weighted average will again be used to approximate the average concentration in the upflow and downflow pipes as

$$
\begin{gathered}
\bar{N}_{n, i}^{L}=\frac{\left(L_{n}-P_{n}\right) N_{n, i}^{\prime}+L_{n}^{a} N_{n, i}^{\prime a}}{L_{n}-P_{n}+L_{n}^{a}} \\
\bar{N}_{n, i}^{D}=\frac{\left(D_{n}-W_{n}\right) N_{n, i}^{\prime \prime}+D_{n}^{a} N_{n, i}^{\prime \prime a}}{D_{n}-W_{n}+W_{n}^{a}}
\end{gathered}
$$

Together with the flow data obtained from the transient flow model developed in Section 8.2, Equations (8.23)-(8.30) determine the concentration gradient across the cascade.

\subsection{Model Implementation}

The two models described in Sections 8.2 and 8.3 have been implemented in a software package collectively named TransCasc. The software package consists of

1. ConcenMS.m - A program written in MATLAB to solve the steady-state, multicomponent gradient equations (section 3.2) to provide the initial conditions for the transient gradient calculation.

2. ConcenMT.m - A program written in MATLAB to solve the transient, multicomponent gradient equations (section 8.3) as a function of flow data obtained 
from the flow model and performance map obtained from a centrifuge model.

3. ConcenSfnc.m - A program written to call ConcenMT.m during the Simscape simulation at each time step.

4. PostPlot.m - A program written in MATLAB to plot the results of the simulation.

5. PostProc.m - A program written in MATLAB to post-process the results of the simulation and save to a .mat file.

6. TC_Main.m - A program written in MATLAB to read the inputs, initialize, and run the simulation.

7. TC_CreateModel.m - A program written in MATLAB to programmatically setup the Simscape model (CaseName.mdl) based on the inputs provided in Input.xlsx.

8. Input.xlsx - A Microsoft Excel spreadsheet designed to serve as the input file for TC_Main.m.

The implementation algorithm of the TransCasc software package is shown in Figure 8.4. Once the inputs are defined and the file is saved, TC_Main.m is executed to initialize, call TC_CreateModel.m to create the Simscape model (CaseName.mdl), and to start the simulation. At each time step, the flow equations are solved then the gradient equations.

The ordinary differential equations that govern the fluid flow and isotope separation in the cascade developed in the previous sections are solved using the standard Runge-Kutta solvers built into the Simulink platform. For this particular application, it is suspected that the equations will be stiff and the default solver used in TransCasc is ode15s. This is an implicit, variable-order type integration routine based on Numerical Differentiation Formulas (NDFs) [112]. The variable-step option is chosen, allowing the time step to decrease when there are rapid changes occurring and increase when slower changes occur. The maximum allowable time step is set to 60 seconds and the maximum relative tolerance is set to $0.1 \%$. Because there are no expected discontinuities, the zero-crossing algorithm is disabled, as this was found to cause convergence to stall. 


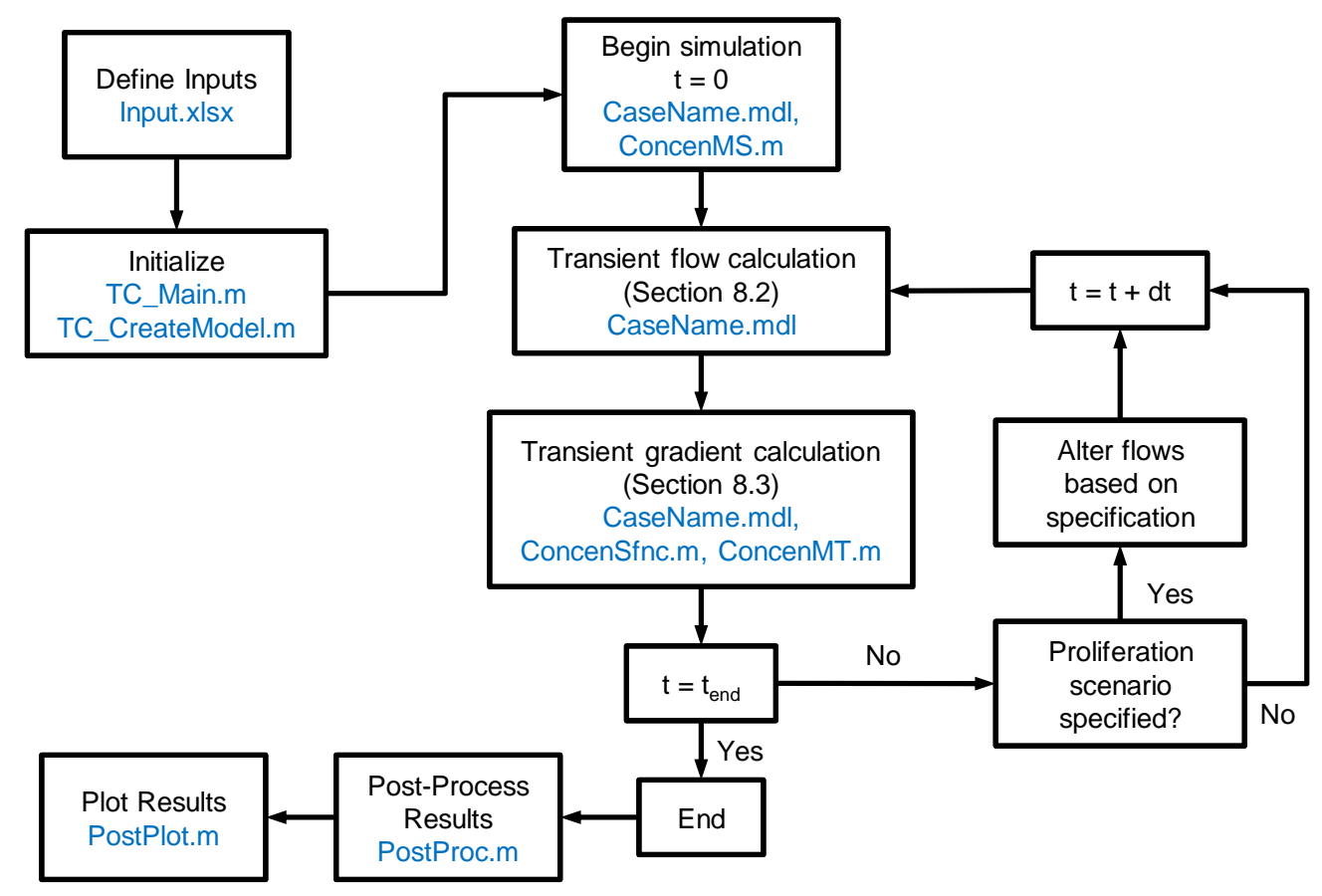

Figure 8.4: Flow chart for a typical TransCasc simulation. The program associated with each step of the simulation is labeled in blue.

\subsubsection{Off-Normal Operation Specification}

The normal operation of the cascade is controlled by specifying either the stage cuts or stage upflow rates along with the cascade feed rate and cascade product rate. To change the operation, several flow controls can be tuned by specifying one or several factors.

\section{Upflow and Cut Scale Factor}

The upflow scale factor $\left(\zeta_{L, n}\right)$ and the cut scale factor $\left(\zeta_{\theta, n}\right)$ are used to control the proportion of flow up and down the cascade. They cannot be used at the same time as defining the upflow rate and stage cut over-defines the problem. The upflow scale factor is defined as

$$
\zeta_{L, n}=\frac{L_{\text {off-normal }, n}}{L_{\text {normal }, n}}
$$

It is assumed that the change to off-normal operation is based on a ramp function 
governed by four specified times (or a step function if the times are chosen as such). The upflow scale factor adjusts the stage upflow rates as

$$
L_{n}= \begin{cases}L_{\mathrm{spec}, n}, & t=0 \cdots t_{1} \\ L_{\mathrm{spec}, n}+\left(\zeta_{L, n}-1\right) \cdot L_{\mathrm{spec}, n} \cdot \frac{t-t_{1}}{t_{2}-t_{2}}, & t=t_{1} \cdots t_{2} \\ \zeta_{L, n} \cdot L_{\mathrm{spec}, n}, & t=t_{2} \cdots t_{3} \\ \zeta_{L, n} \cdot L_{\mathrm{spec}, n}-\left(\zeta_{L, n}-1\right) \cdot L_{\mathrm{spec}, n} \cdot \frac{t-t_{3}}{t_{4}-t_{3}}, & t=t_{3} \cdots t_{4} \\ L_{\mathrm{spec}, n}, & t=t_{4} \cdots t_{\mathrm{end}}\end{cases}
$$

where $L_{\mathrm{spec}, n}$ is the specified normal upflow rate in each stage, $t_{1}$ is the initial time to start the off-normal operation, $t_{2}-t_{1}$ is the time period of the ramp function to off-normal operation, $t_{3}$ is the specified time to return to normal operation and $t_{4}-t_{3}$ is the time period of the ramp function to return to normal operation. If $t_{2}=t_{1}$ and $t_{4}=t_{3}$ then a step function is specified. The cut scale factor is defined as

$$
\zeta_{\theta, n}=\frac{\theta_{\text {off-normal }, n}}{\theta_{\text {normal }, n}}
$$

and controls the specified cut as

$$
\theta_{n}= \begin{cases}\theta_{\mathrm{spec}, n}, & t=0 \cdots t_{1} \\ \theta_{\mathrm{spec}, n}+\left(\zeta_{\theta, n}-1\right) \cdot \theta_{\mathrm{spec}, n} \cdot \frac{t-t_{1}}{t_{2}-t_{2}}, & t=t_{1} \cdots t_{2} \\ \zeta_{\theta, n} \cdot \theta_{\mathrm{spec}, n}, & t=t_{2} \cdots t_{3} \\ \zeta_{\theta, n} \cdot \theta_{\mathrm{spec}, n}-\left(\zeta_{\theta, n}-1\right) \cdot \theta_{\mathrm{spec}, n} \cdot \frac{t-t_{3}}{t_{4}-t_{3}}, & t=t_{3} \cdots t_{4} \\ \theta_{\mathrm{spec}, n}, & t=t_{4} \cdots t_{\mathrm{end}}\end{cases}
$$

where $\theta_{\mathrm{spec}, n}$ is the specified normal stage cut in each stage. Both of these function are illustrated in Figure 8.5a 


\section{Upflow Withdrawal Rate Factor}

The upflow withdrawal rate is defined as the amount of material withdrawn from the stage upflows. A positive value indicates the withdrawal of material, a negative value indicates the addition of material. The specified upflow withdrawal rate $\left(\zeta_{P, n}\right)$ controls the stage product withdrawal rate as

$$
P_{n}= \begin{cases}0, & t=0 \cdots t_{1} \\ \zeta_{P, n} \cdot \frac{t-t_{1}}{t_{2}-t_{2}}, & t=t_{1} \cdots t_{2} \\ \zeta_{P, n}, & t=t_{2} \cdots t_{3} \\ \zeta_{P, n}-\zeta_{P, n} \cdot \frac{t-t_{3}}{t_{4}-t_{3}}, & t=t_{3} \cdots t_{4} \\ 0, & t=t_{4} \cdots t_{\mathrm{end}}\end{cases}
$$

and is illustrated in Figure 8.5b.

\section{Cascade Feed Rate Factor}

The cascade feed rate factor specifies an increase in the cascade feed rate and is illustrated in Figure 8.5c. A positive value indicates an increase in cascade feed rate and a negative value indicates a decrease in cascade feed rate. The cascade feed rate factor is defined as

$$
\zeta_{F}=\frac{F_{\text {off-normal }}-F_{\text {spec }}}{F_{\text {spec }}}
$$

and controls the cascade feed rate as 


$$
F_{n F}= \begin{cases}F_{\mathrm{spec}}, & t=0 \cdots t_{1} \\ F_{\mathrm{spec}}+\zeta_{F} \cdot F_{\mathrm{spec}} \cdot \frac{t-t_{1}}{t_{2}-t_{2}}, & t=t_{1} \cdots t_{2} \\ \left(1+\zeta_{F}\right) \cdot F_{\mathrm{spec}}, & t=t_{2} \cdots t_{3} \\ \left(1+\zeta_{F}\right) \cdot F_{\mathrm{spec}}-\zeta_{F} \cdot F_{\mathrm{spec}} \cdot \frac{t-t_{3}}{t_{4}-t_{3}}, & t=t_{3} \cdots t_{4} \\ F_{\mathrm{spec}}, & t=t_{4} \cdots t_{\mathrm{end}}\end{cases}
$$

$F_{\text {spec }}$ is the specified normal cascade feed rate.

\section{Centrifuge Scale Factor}

The centrifuge scale factor dictates the increase of centrifuges in a stage and is shown in Figure $8.5 \mathrm{~d}$. The centrifuge scale factor is defined as

$$
\zeta_{M, n}=\frac{M_{\mathrm{off}-\mathrm{normal}, n}}{M_{\mathrm{normal}, n}}
$$

It is assumed that the addition or loss of a centrifuge is instantaneous and therefore described by a step function as

$$
M_{n}= \begin{cases}M_{\mathrm{spec}, n}, & t=0 \cdots t_{1} \\ M_{\mathrm{spec}, n}+\zeta_{M, n}, & t=t_{1} \cdots t_{2} \\ M_{\mathrm{spec}, n}, & t=t_{2} \cdots t_{\mathrm{end}}\end{cases}
$$

where $M_{\mathrm{spec}, n}$ is the specified normal number of centrifuges in each stage, $t_{1}$ is the specified time to start off-normal operation and $t_{2}$ is the specified time to return to normal operation. 


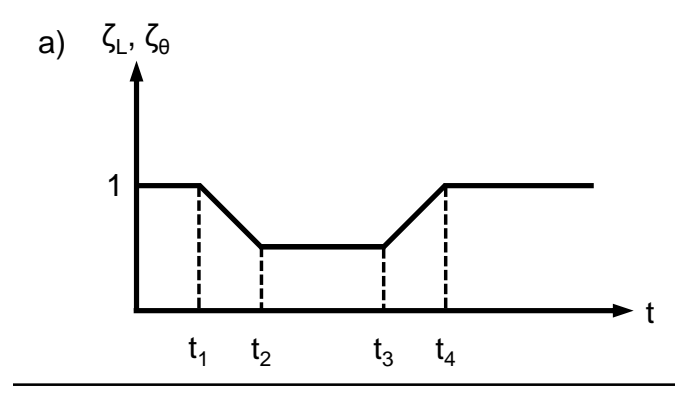

c)

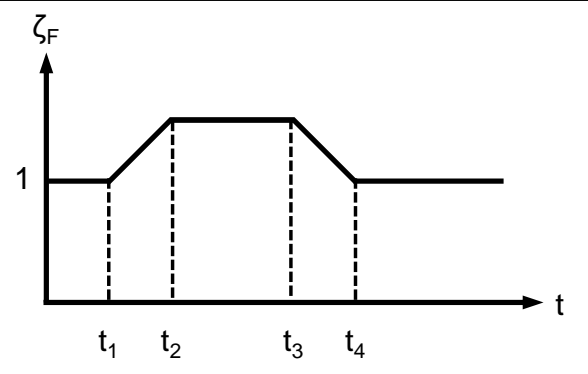

b)

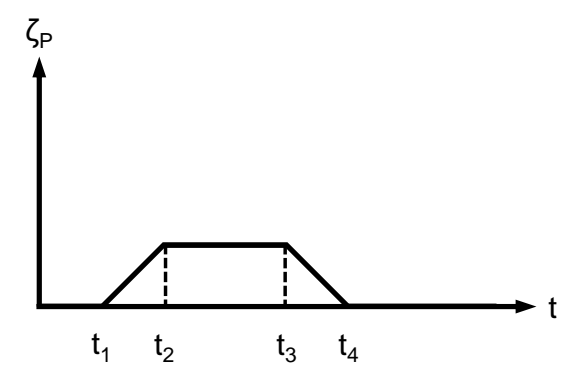

d)

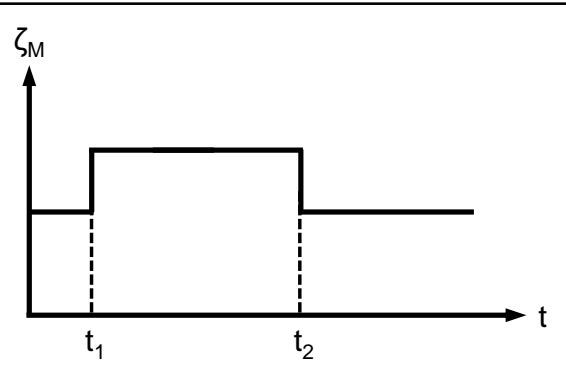

Figure 8.5: Off-normal operation flow specifications in TransCasc, a) Upflow and cut scale factor, b) Upflow withdrawal rate factor, c) Cascade feed rate factor, d) Centrifuge scale factor. The upflow and cut scale factor are used to control the stage flow rates. They cannot be used together as this would over-define the simulation. The upflow withdrawal rate factor controls the amount of material withdrawn from the stage upflow. The cascade feed rate factor controls the cascade feed rate. The centrifuge scale factor controls the number of centrifuges in each stage.

\subsection{Model Verification}

In order to verify that TransCasc is working correctly and that the assumptions made are appropriate, two verification cases are performed. In the first case, the proper number of segments needed in the lumped pipe model is examined. In the second case, an off-normal simulation is compared with an existing model in the literature.

\subsubsection{Lumped Pipe Model}

To determine the appropriate number of pipes to choose in the lumped pipe model, an example off-normal simulation is performed with the Rome3 cascade (which will be described later in the chapter). This cascade has the greatest holdup amongst the other developed cascades and should be the most effected by the selected number of pipe 

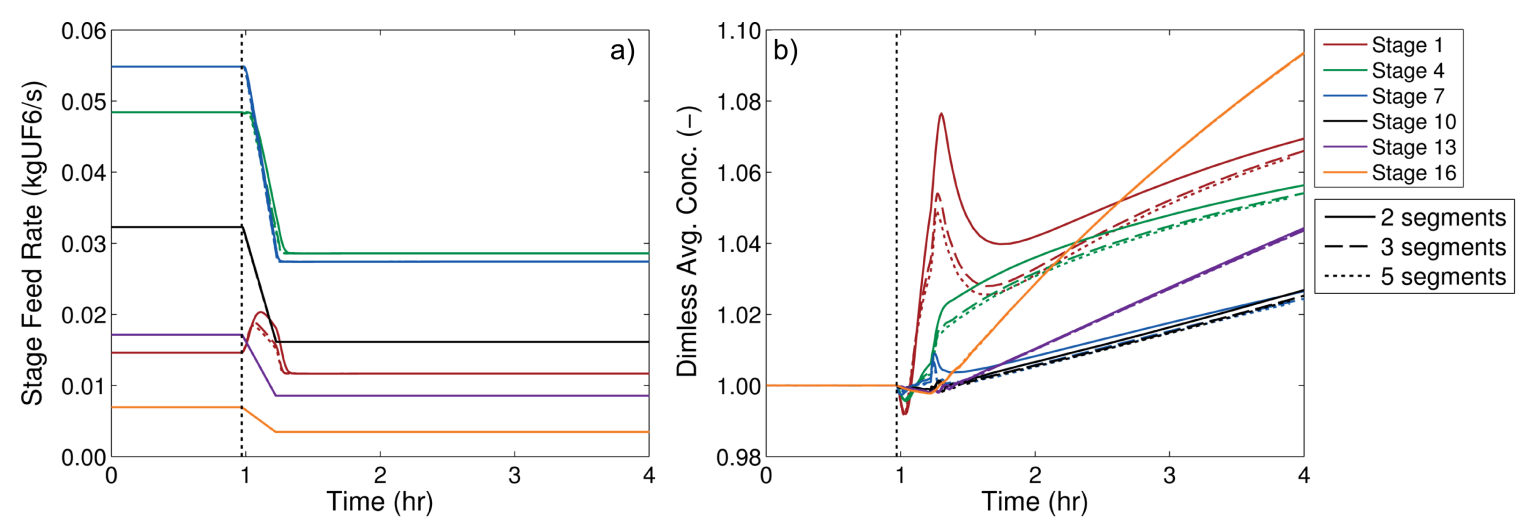

Figure 8.6: Results of the lumped pipe model verification study. A simulation is run where the stage upflow rates are reduced by half over a fifteen minute period. The stage feed rates a) and average stage concentrations b) are compared for cases where 2, 3, and 5 segments are used in the pipe model. The verification study shows that 3 segments are sufficient.

segments. The simulation procedure is as follows:

1. Run the cascade at normal conditions for one hour

2. Specify an upflow scale factor for each stage of 0.5 over a period of 15 minutes

3. After 50 hours, change the upflow scale factor to 1.0 over a period of 15 minutes

The total simulation time is set to 100 hours and lumped pipe models of 2,3 , and 5 segments are chosen. The run time for each case is 786.6, 856.5, and 1104.7 seconds, respectively. Figure 8.6 shows the stage feed rates and normalized average stage concentrations. The normalized average stage concentration is the ratio of the stage concentration at a given time to the normal operation concentration. The figure shows that the bottom stage of the cascade is the most effected by the number of segments chosen. This is due to the surge of flow that travels down the cascade when the upflow rates are decreased. All segment cases converge to the same off-normal operation, but the time and peak values vary. Based on the results, 2 pipe segments is too few, but because 3 segments and 5 compare well, 3 segments is the appropriate choice. 
Table 8.1: Cascade and centrifuge parameters used in comparison study to the Rousseau model

\begin{tabular}{rccc}
\hline Parameter & Variable & Unit & Value \\
\hline Cascade Feed Rate & $F$ & $\mathrm{kgU} / \mathrm{yr}$ & 1108 \\
Centrifuge Wall Speed & $v_{a}$ & $\mathrm{~m} / \mathrm{s}$ & 700 \\
Centrifuge Length & $H$ & $\mathrm{~m}$ & 1.67 \\
Centrifuge Diameter & $2 a$ & $\mathrm{~m}$ & 0.1829 \\
Pipe Diameter & $D_{p}$ & $\mathrm{~m}$ & 0.0762 \\
Centrifuge Wall Pressure & $p_{w}$ & torr & 100 \\
Centrifuge Average Temperature & $T_{0}$ & $\mathrm{~K}$ & 320 \\
Pipe Aggregate Length Factor & $F_{p a}$ & - & 180,000 \\
\hline
\end{tabular}

\subsubsection{Flow Model}

To ensure that the flow model is working correctly and to choose the appropriate characterizing factors (pipe length factor and pipe aggregate length factor), a verification case is performed by comparing to the model of Rousseau et. al. [93]. The cascade and centrifuge details derived from Rousseau's study is shown in Table 8.1. The number of centrifuges per stage and upflow rates are shown in Table 8.2. The upflow rates shown here have been extracted from a graph in Rousseau's presentation using DataThief III.

To compare with Rousseau's off-normal simulation, the cascade is run normally for one minute, then the cascade feed flow rate is increased by $10 \%$ over a one minute period. This off-normal operation is held for 20 minutes, then the cascade is returned to normal operating conditions over a one minute period. For this cascade, the combination of pipe diameter and pipe aggregate length factor given in Table 8.1 yields a pressure differential across the cascade of roughly 10 torr, corresponding with Rousseau's results. Five cases are defined by varying the pipe length factor: Case $1-F_{p}=3$, Case $2-F_{p}=2$, Case $3-$ $F_{p}=1$, Case $4-F_{p}=0.5$, Case $5-F_{p}=0.25$

Figure 8.7 shows the predicted time variation of the cascade tails rate and feed stage upflow rate due to the increase in cascade feed rate. The "stepping" behavior of the 
Table 8.2: Stages, number of centrifuges, and upflow rates specified in verification study

\begin{tabular}{ccc}
\hline Stage & Centrifuges & $\begin{array}{c}\text { Upflow Rate } \\
(\mathrm{kgU} / \mathrm{yr})\end{array}$ \\
\hline $1 \mathrm{~S}$ & 6 & 835 \\
$2 \mathrm{~S}$ & 12 & 1564 \\
$3 \mathrm{~S}$ & 17 & 2180 \\
$4 \mathrm{~S}$ & 21 & 2699 \\
$1 \mathrm{E}$ & 24 & 3138 \\
$2 \mathrm{E}$ & 20 & 2579 \\
$3 \mathrm{E}$ & 16 & 2090 \\
$4 \mathrm{E}$ & 13 & 1695 \\
$5 \mathrm{E}$ & 10 & 1328 \\
$6 \mathrm{E}$ & 8 & 1043 \\
$7 \mathrm{E}$ & 6 & 785 \\
$8 \mathrm{E}$ & 5 & 582 \\
$9 \mathrm{E}$ & 3 & 392 \\
$10 \mathrm{E}$ & 2 & 257 \\
$11 \mathrm{E}$ & 1 & 122 \\
\hline
\end{tabular}

Rousseau results is an artifact of acquiring the data through Data Thief III. Ideally, the two models should predict the same values for the steady-state values of normal and offnormal operation with the only difference being the transition time between the two states. The reason for the difference here is that the values of stage cut chosen by Rousseau is not known exactly and was taken from a plot using Data Thief III. Even though the values do not match exactly, the time evolution is quite similar and shows that a choice of pipe length factor between 0.5 and 2 is appropriate. The run time for each of these TransCasc simulations is roughly 200 seconds compared to approximately 30 minutes for Rousseau's model [93].

\subsection{Case Summaries}

To gain a true understanding of signatures of misuse in a cascade and proliferation timeframes, a number of cascades should be considered to determine if cascade structure and 

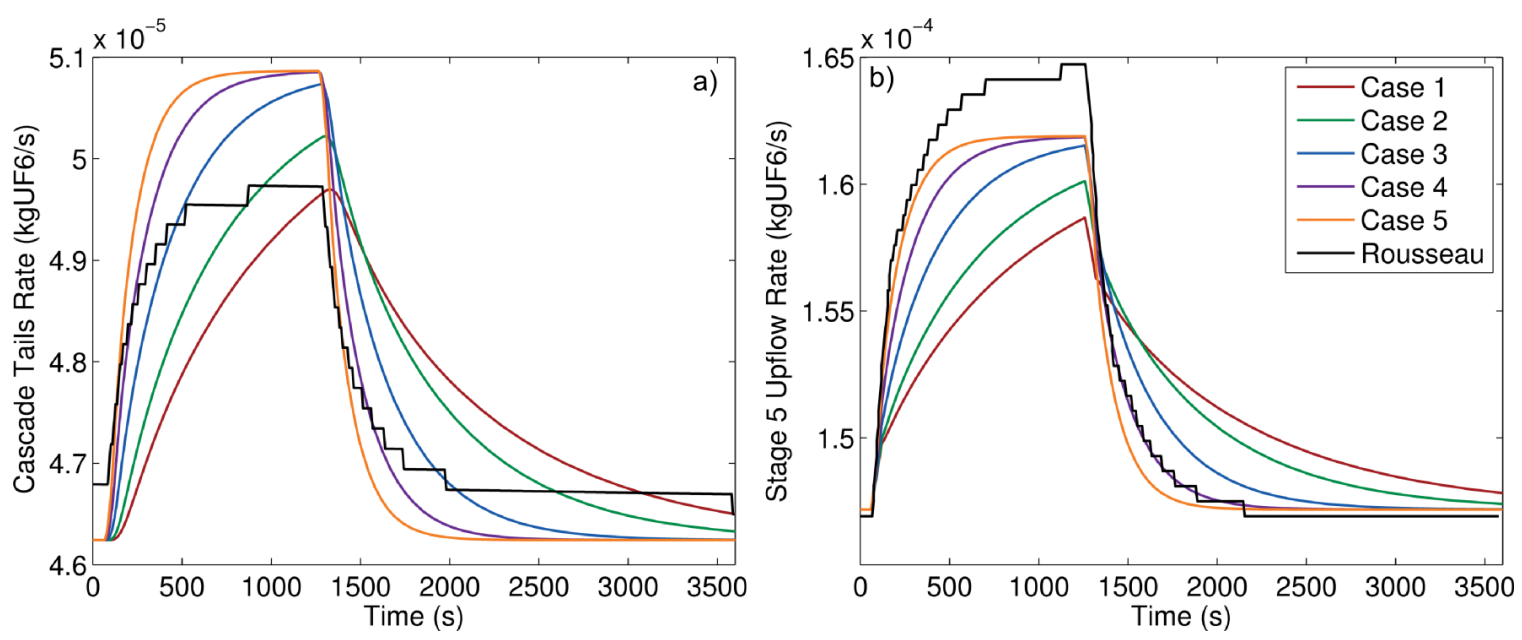

Figure 8.7: Results of the flow model verification study. The cascade feed rate is increased by $10 \%$ over a one minute period. The cascade tails rate a) and the Stage 5 upflow rates b) are compared with data from a model developed by Rousseau et. al. The results of the verification show good agreement in the transient response. The difference between normal and off-normal flow rates is a result of retrieving the data from a plot using Data Thief III.

centrifuge type have an effect. Five cascades are developed with two different centrifuges (Rome and Iguaçu) and varying nominal cascade separative powers. The nominal cascade designs are shown in Table 8.3 and are classified by the type of centrifuge. The performance of the centrifuges are calculated by the Pancake code. For the Iguaçu cascades, there are 19 total stages with 14 enriching and 5 stripping stages. The Rome cascades consist of 18 total stages with 13 enriching stages and 5 stripping stages. The nominal cascade separative power ranges from 1,000-100,000 SWU/yr for the Iguaçu cascades and 10,000100,000 SWU/yr for the Rome cascades. The 1,000 SWU/yr Rome cascade was dropped because the number of centrifuges needed per stage is too few to create a physically relevant cascade. The cascade feed concentration is assumed to be commercial grade natural uranium. The combination of pipe diameter, pipe length factor, and pipe aggregate length factor were chosen to keep the pressure drop across the cascade near 10 torr. The full stage details of each cascade are given in Appendix B.

It is expected that different off-normal scenarios will produce different variations to the cascade external parameters and the transition between normal and off-normal states 
Table 8.3: Nominal cascade designs for transient study

\begin{tabular}{lcccccc}
\hline Parameter & Unit & Iguaçu1 & Iguaçu2 & Iguaçu3 & Rome2 & Rome3 \\
\hline Cascade Feed Rate & $\mathrm{kgU} / \mathrm{yr}$ & 2,099 & 20,990 & 209,900 & 18,630 & 186,300 \\
Cascade Feed Concentration & at. frac. & 0.0072 & 0.0072 & 0.0072 & 0.0072 & 0.0072 \\
Target Product Concentration & at. frac. & 0.0350 & 0.0350 & 0.0350 & 0.0350 & 0.0350 \\
Target Tails Concentration & at. frac. & 0.0035 & 0.0035 & 0.0035 & 0.0035 & 0.0035 \\
Number of Enriching Stages & - & 14 & 14 & 14 & 13 & 13 \\
Number of Stripping Stages & - & 5 & 5 & 5 & 5 & 5 \\
Total Number of Stages & - & 19 & 19 & 19 & 18 & 18 \\
Nominal Separative Power & $\mathrm{SWU} / \mathrm{yr}$ & 1,000 & 10,000 & 100,000 & 10,000 & 100,000 \\
Centrifuges (Ideal) & - & 226.59 & $2,265.90$ & $22,658.98$ & 186.53 & $1,865.28$ \\
Centrifuges (Rounded) & - & 228 & 2,268 & 22,661 & 186 & 1,865 \\
Pipe Diameter & $\mathrm{m}$ & 0.0762 & 0.1143 & 0.1524 & 0.1143 & 0.1524 \\
Pipe Length Factor & - & 1.0 & 1.0 & 1.0 & 1.0 & 1.0 \\
Pipe Aggregate Length Factor & - & 90000 & 4500 & 15 & 4500 & 5 \\
Reynolds Number Range & - & $10-400$ & $70-2300$ & $500-17000$ & $60-1900$ & $450-14000$ \\
Pressure Drop & torr & 13 & 16 & 14 & 8 & 5 \\
\hline
\end{tabular}

will also be different. Four different scenarios are developed with the verification goals of the IAEA in mind. Table 8.4 shows these scenarios, cases, and mathematical specification applied in TransCasc.

The four scenarios represent intentional misuse with and without an operator trying to mask diversion and unintentional off-normal operation. The first scenario, centrifuge failure in the feed stage represents an unintentional off-normal operation of the cascade. It is assumed that at some time during the normal operation of the cascade, a certain number of centrifuges in the feed stage fails and they are immediately isolated from the cascade by either shutting a valve or crimping piping. The second scenario is an intentional overproduction of material by adding natural uranium to the upflow of the top stripping stage. The third scenario is the same at the second scenario but with the withdrawal of material from the top and bottom of the cascade in an effort to mask the overproduction. The fourth scenario is the increase and decrease of the stage upflow rates. This scenario is used to produce extra product at a lower enrichment or less product at a higher enrichment.

For each case, the simulation time is set to 50 hours and the cascade is run at normal conditions for one hour. Next, the off-normal specification is enacted over a 15 minute 
Table 8.4: Simulation cases and off-normal specifications

\begin{tabular}{lcl}
\hline Scenario & Case & Specification \\
\hline & 1 & $\zeta_{M, n F}=-\operatorname{ceil}\left(0.01 \cdot M_{n F}\right)$ \\
Centrifuge failure in the feed stage & 2 & $\zeta_{M, n F}=-\operatorname{ceil}\left(0.05 \cdot M_{n F}\right)$ \\
& 3 & $\zeta_{M, n F}=-\operatorname{ceil}\left(0.10 \cdot M_{n F}\right)$ \\
& 4 & $\zeta_{M, n F}=-\operatorname{ceil}\left(0.20 \cdot M_{n F}\right)$ \\
\hline Addition of undeclared material into top & 5 & $\zeta_{P, n F-1}=-0.005 \cdot L_{n F-1}$ \\
stripping stage upflow & 6 & $\zeta_{P, n F-1}=-0.01 \cdot L_{n F-1}$ \\
\hline & & $\zeta_{P, n F-1}=-0.005 \cdot L_{n F-1}$ \\
Addition of undeclared material into top & 7 & $\zeta_{P, n P}=-0.083 \cdot \zeta_{P, n F-1}$ \\
stripping stage upflow and withdrawal at top & & $\zeta_{P, 1}=\zeta_{P, n F-1}-\zeta_{P, n P}$ \\
and bottom of cascade & 8 & $\zeta_{P, n F-1}=-0.01 \cdot L_{n F-1}$ \\
& & $\zeta_{P, 1}=\zeta_{P, n F-1}-\zeta_{P, n P}$ \\
\hline & 9 & $\zeta_{L, n}=1.25$ \\
Increase and decrease stage upflow rates & 10 & $\zeta_{L, n}=1.05$ \\
& 11 & $\zeta_{L, n}=0.95$ \\
& 12 & $\zeta_{L, n}=0.75$ \\
\hline
\end{tabular}

period. When the simulation reaches 26 hours, the off-normal specification is removed over a period of 15 minutes.

\subsection{Simulation Results}

In this section, the results of the off-normal simulations are presented. The cascade external flow rates and concentrations are presented in a normalized fashion where a particular parameter is non-dimensionalized by its normal operational value. The transition from normal operating conditions to off-normal operating conditions will be referred to as N-to$\mathrm{ON}$ and the transition from off-normal conditions to normal conditions will be referred to as ON-to-N.

\subsubsection{Signatures of Misuse in a Cascade}

Figures 8.8 and 8.9 show the flow rate and concentration of ${ }^{235} \mathrm{U}$ in the product and tails streams for the Iguaçu2 cascade. For brevity, this cascade will serve as a representative 

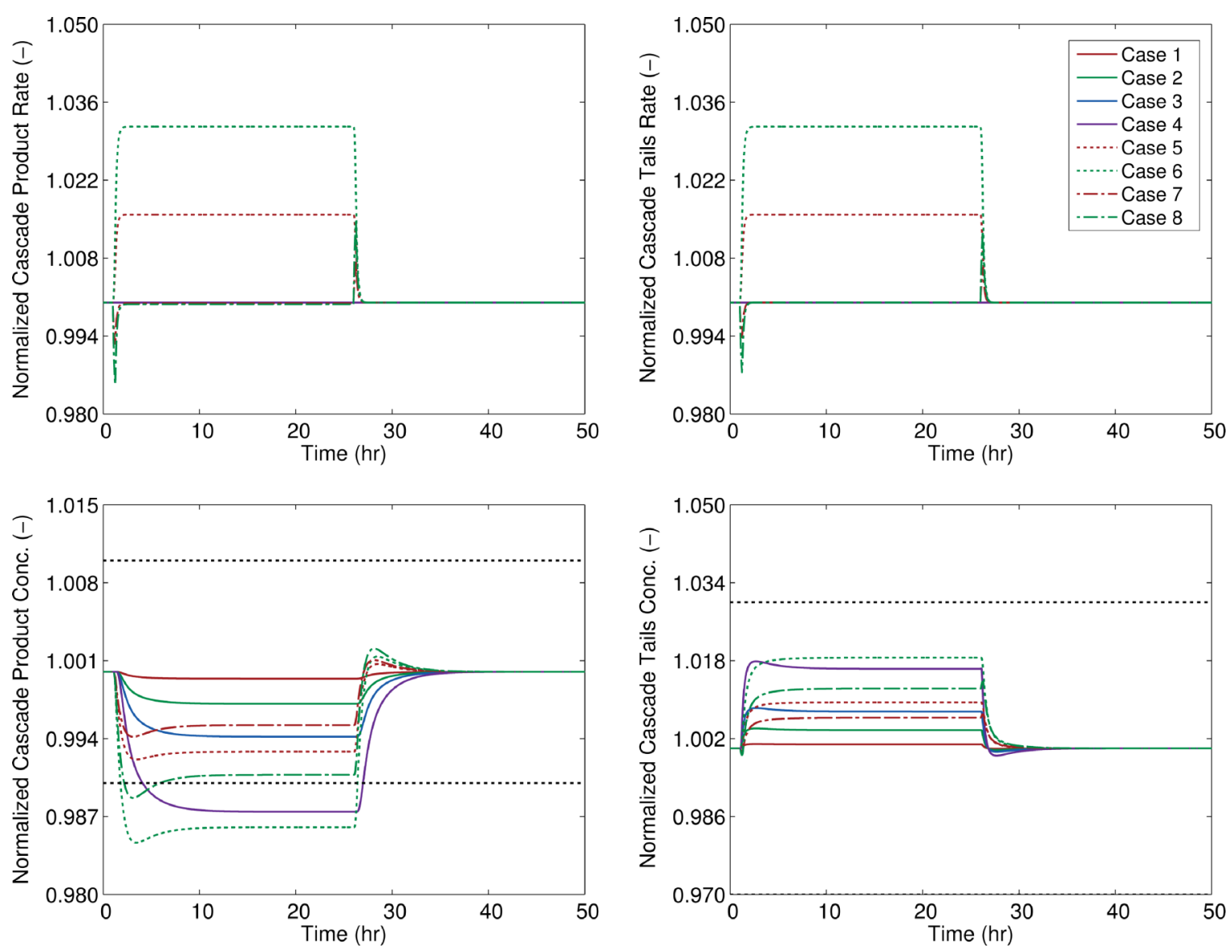

Figure 8.8: Cascade product and tails rates and concentrations for the Iguaçu2 cascade, Cases 1-8. The values are normalized by the normal cascade rates and concentrations. Cases 1-4, centrifuge failure in the feed stage, represent unintentional off-normal operation of the cascade. Cases 5-8 represent intentional misuse of the cascade by introducing undeclared feed. A clear difference is shown in the cascade dynamics between the two scenarios.

case for the other cascades because there is very little qualitative difference between all of the cascades. Because the changes in enrichment in Cases 9-12 are much larger than 1-8, the results are separated over two graphs. Smith and Lebrun [29] studied the feasibility of an on-line enrichment monitor for use in an unattended monitoring system. They set target relative uncertainties for the enrichment measurement at $1 \%, 2 \%$, and $3 \%$ for the product, feed, and tails streams respectively. These targets are shown on the concentration plots as a black, dashed line.

Figure 8.8 shows the normalized product and tails rates and concentrations as a function 

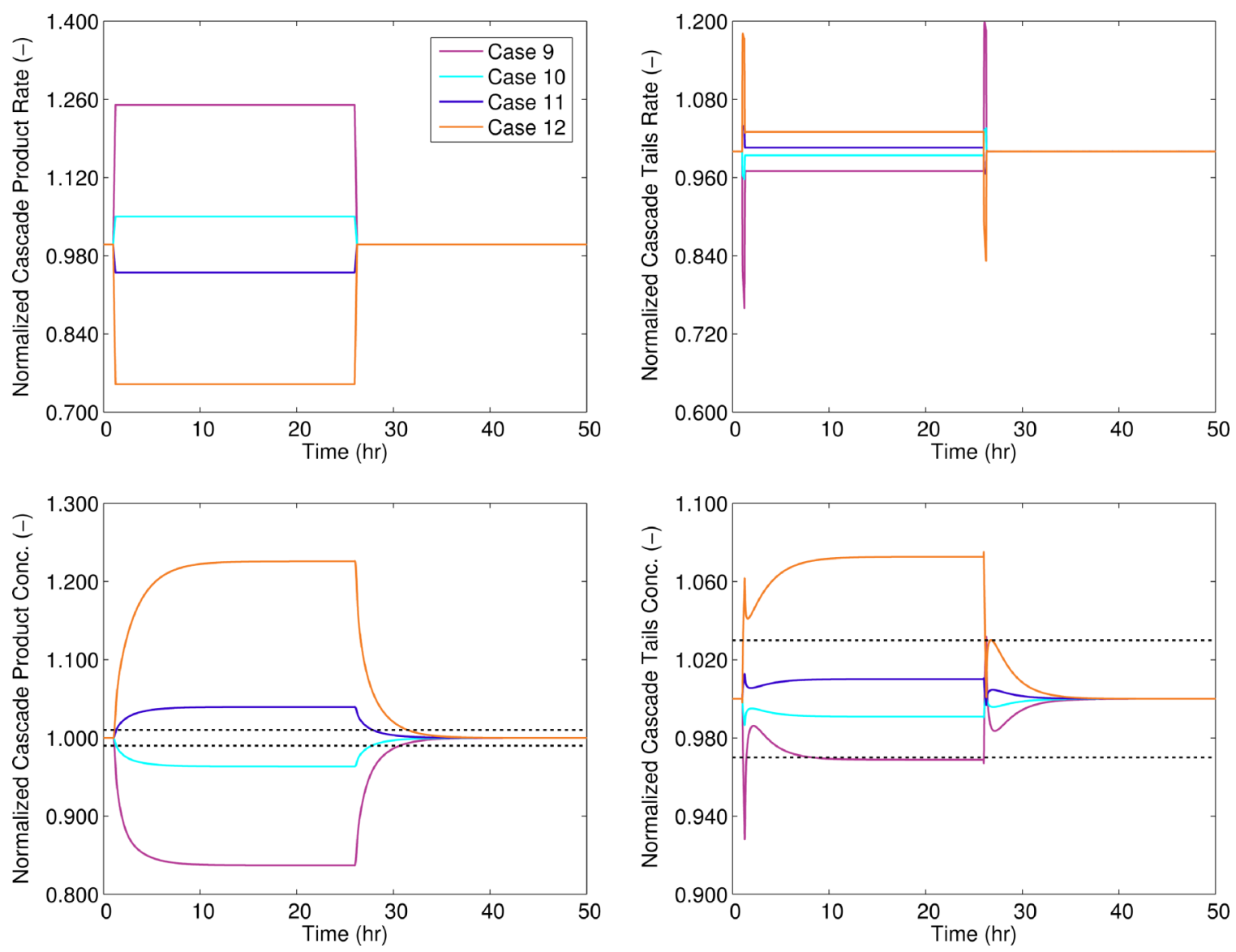

Figure 8.9: Cascade product and tails rates and concentrations for the Iguaçu2 cascade, Cases 9-12. The values are normalized by the normal cascade rates and concentrations. These cases represent scenarios that should be detected by unattended monitoring systems as the change in the concentrations is large.

of time for Cases 1-8. For the first four cases, the flow rates in the cascade do not change and the failure of centrifuge causes a smooth decrease in the cascade product concentration with a slight overshoot in the cascade tails concentration. As expected the greater the number of centrifuges that fail, the greater the decrease in cascade product concentration and increase in cascade tails concentration. For the addition of undeclared feed material (Cases 5-6), the cascade product and tails rates increase as expected. This should be a case that is detectable by cylinder weight monitoring and mass balances. For the concentrations, the opposite of the centrifuge failure cases is found. The cascade product concentration decreases with a slight overshoot of the steady off-normal value and the cascade tails concentration gradually and smoothly transitions to the off-normal value. For the addition 

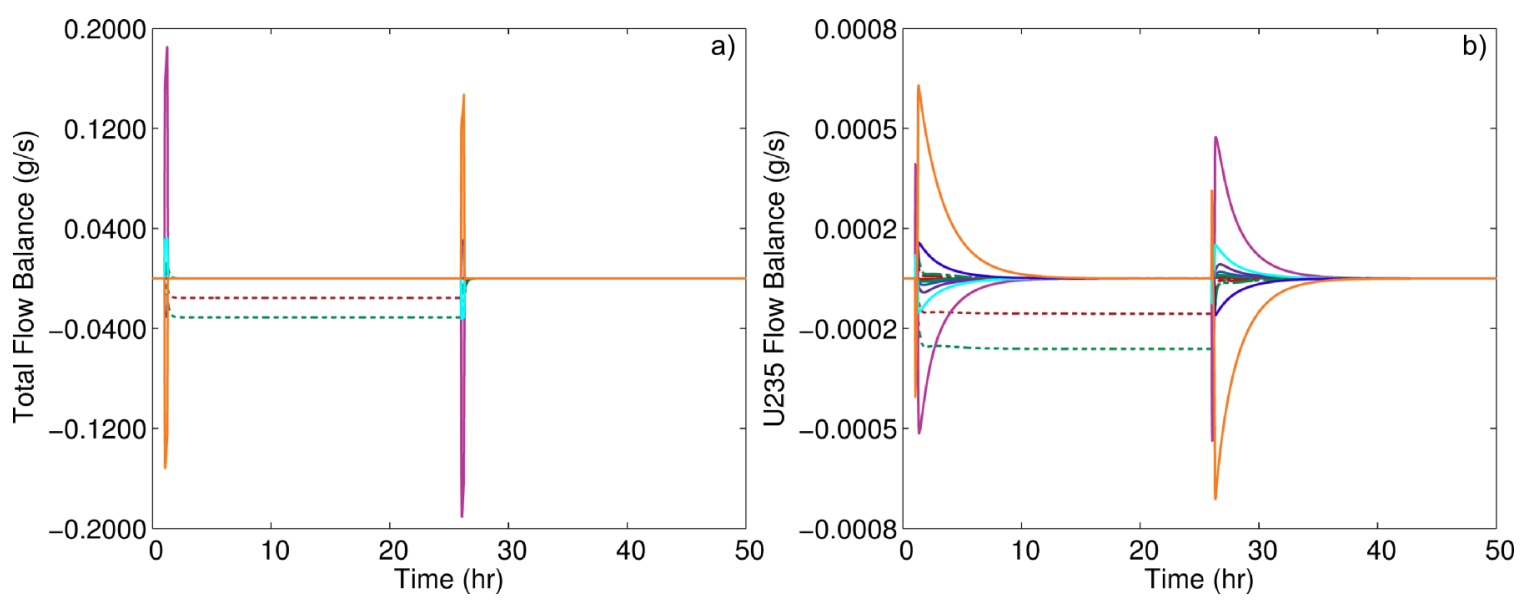

Figure 8.10: Instantaneous flow balances for the Iguaçu2 cascade, a) Total flow balance, b) ${ }^{235} \mathrm{U}$ flow balance. The imbalance of the flow rates is relatively short while the imbalance of ${ }^{235} \mathrm{U}$ requires significantly more time to dissipate. As expected, Cases 5-6 show a constant imbalance due to the undeclared feed.

and withdrawal of undeclared material (Cases 7-8), the withdrawal rates are carefully chosen such that the cascade product and tails rates remain at the normal operational value. However, a sharp decrease in the flow rates occurs before returning to the normal operating value. The withdrawal of material also serves to reduce the change in cascade product and tails concentrations. This may be a scenario that is difficult to detect as the peak concentrations are within the uncertainty bounds, but the sharp decrease in the cascade external flow rates may serve as a indicator of this type of misuse.

The increase and decrease of the stage upflow rates (Cases 9-12), shown in Figure 8.9 are cases that should certainly be discovered by enrichment monitoring and cylinder weighing due to the large variation in flow rate and concentrations.

The instantaneous total and ${ }^{235} \mathrm{U}$ flow balances are shown in Figure 8.10. For the cases of intentional misuse, there is a short pulse of total flow imbalance and a large increase and gradual decrease in the ${ }^{235} \mathrm{U}$ flow balance. The time to achieve a flow balance of zero is much longer in the extreme flow alteration cases (Cases 9 and 12). As expected, Cases 5 and 6 show a signature of the addition of undeclared feed due to the steady unbalance in the cascade external flows.

One significant difference between the external streams in misuse cases versus 

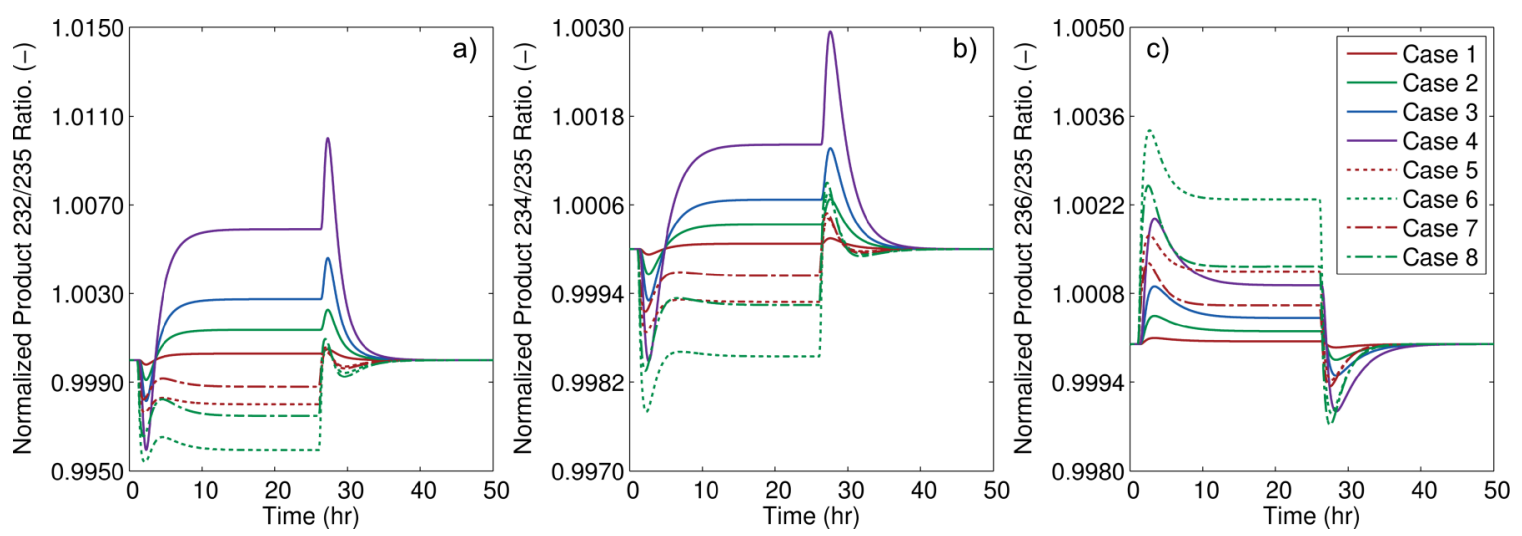

Figure 8.11: Normalized ratio of the minor isotopes in the cascade product stream to ${ }^{235} \mathrm{U}$, a) ${ }^{232} \mathrm{U}$, b) ${ }^{234} \mathrm{U}$, c) ${ }^{236} \mathrm{U}$. The difference between the intentional misuse cases and centrifuge failure is clearly seen in the behavior of the lighter minor isotopes $\left({ }^{232} \mathrm{U}\right.$ and $\left.{ }^{234} U\right)$.

unintentional off-normal operation shows up in the normalized ratio of the minor isotopes to ${ }^{235} \mathrm{U}$ shown in Figure 8.11. This signature is most prevalent in the lighter isotopes $\left({ }^{232} \mathrm{U}\right.$ and $\left.{ }^{234} \mathrm{U}\right)$. In the case of centrifuge failure, there is an initial decrease in the ratio followed by a gradual increase greater than the normal operational level. For the cases of misuse, there is an initial decrease in the ratio that steadily remains less than the normal ratio. This behavior is not the same for the ${ }^{236} \mathrm{U}$ isotope. This result indicates that the ratio of the light minor isotopes to ${ }^{235} \mathrm{U}$ can be a good indicator of misuse in a cascade. It should be noted that the variations in these ratios are quite small and are within the target uncertainties of the on-line enrichment monitor discussed by Smith and Lebrun [29]. However, in higher pressure cases, Smith and Lebrun were able to find uncertainties near $0.2 \%$ which is capable of detecting the change in ${ }^{232} \mathrm{U}$ ratio. Figure 8.12 shows the behavior of the tails ratios. Case 1-8 product similar responses in the isotopic ratios and do not offer any significant clue as to the type of off-normal operation.

\subsubsection{Off-Normal Operation Time Frames}

Knowledge of the holdup time between two operational states of the cascade is important in two situations. First, for a fully functioning unattended monitoring system the knowledge 

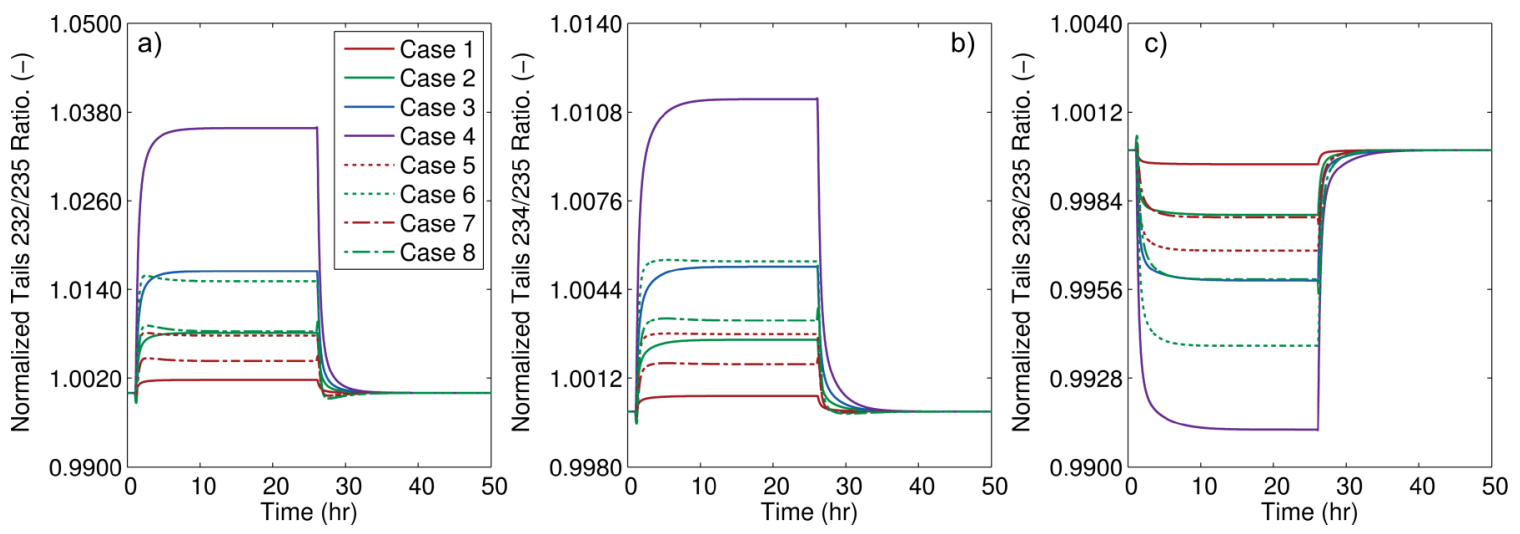

Figure 8.12: Normalized ratio of the minor isotopes in the cascade tails stream to ${ }^{235} \mathrm{U}$, a) $\left.\left.{ }^{232} \mathrm{U}, \mathrm{b}\right){ }^{234} \mathrm{U}, \mathrm{c}\right){ }^{236} \mathrm{U}$. The minor isotope ratios in the tails stream do not differ significantly between the off-normal scenarios.

of the time it takes to go from a normal state to an off-normal state can be used to set the measurement schedule. In the situation when a unattended monitoring system is not installed or has failed, it is important to know how long detectable phenomena remains in the cascade. In the event that a short notice random inspection is needed, it is important to know if the transition time for a particular scenario falls inside or outside the notification time-frame.

Figure 8.13 shows the transition time of N-to-ON state change (top three plots) and ON-to-N state change (bottom three plots) for Cases 1-12 and all cascades. The transition time is determined as the time it takes for the ${ }^{235} \mathrm{U}$ flow balance to reach a new steady value. The following trends are shown in the results:

- The more extreme the off-normal specification (i.e. greater number of failing centrifuges or more upflow withdrawn), the greater the transition time for both $\mathrm{N}$ to-ON and $\mathrm{ON}$-to-N. A few exceptions are found, but this is generally the rule.

- The transition time is a function of both cascade size (i.e. number of centrifuges or nominal separative power) and cascade length (18 vs. 19 stages). For cascades of equal capacity, the longer the cascade, the greater the transition time.

- For Cases 9-12, the transition time for N-to-ON is roughly equal between equal changes to the upflow scale factor $( \pm 0.05$ and \pm 0.25$)$. In the $\mathrm{ON}$-to-N transition, the 
+0.05 case takes longer than the -0.05 case for most cascades.

For many of the cases, the transition time is relatively short, less than 5 hours, and may effect an enrichment measurement. For example, Friend [117] and Ianakiev et. al. [21] use a count rate time of two hours to measure the enrichment in a header pipe. In many cases, this may not be quick enough and a signature of misuse might go unnoticed. For many of the cascades, the transition time for some of the modest off-normal cases (Cases 1, 5, and 7) are on the order of this measurement time. It is encouraging that the transition time is greater than two hours for most cases, allowing detectable phenomena to remain in the cascade long enough to be discovered by destructive assay sampling during short notice random inspections.

\subsection{Summary}

In this chapter, a transient fluid dynamics and isotope separation model of a generic gas centrifuge cascade is developed. These models are implemented in a software package called TransCasc which is developed in MATLAB and Simscape. Two verification studies are performed to ensure the code it working properly and that the simplifications made in the flow model are acceptable. Good agreement is found with another, more sophisticated flow model in the literature for a test case.

The code is used to study off-normal operation of several cascades to determine signatures of misuse and to quantify transition times between normal and off-normal operating states. The results of this study show clear, key differences in the predicted enrichment profiles due to unintentional off-normal operation versus those that might arise from proliferant activities. It is also shown that measurement time of an on-line enrichment monitor may be on the same time scale as some of the presented transition cases. For most cases, detectable phenomena remain in the cascade long enough to be sampled during a short notice inspection. 

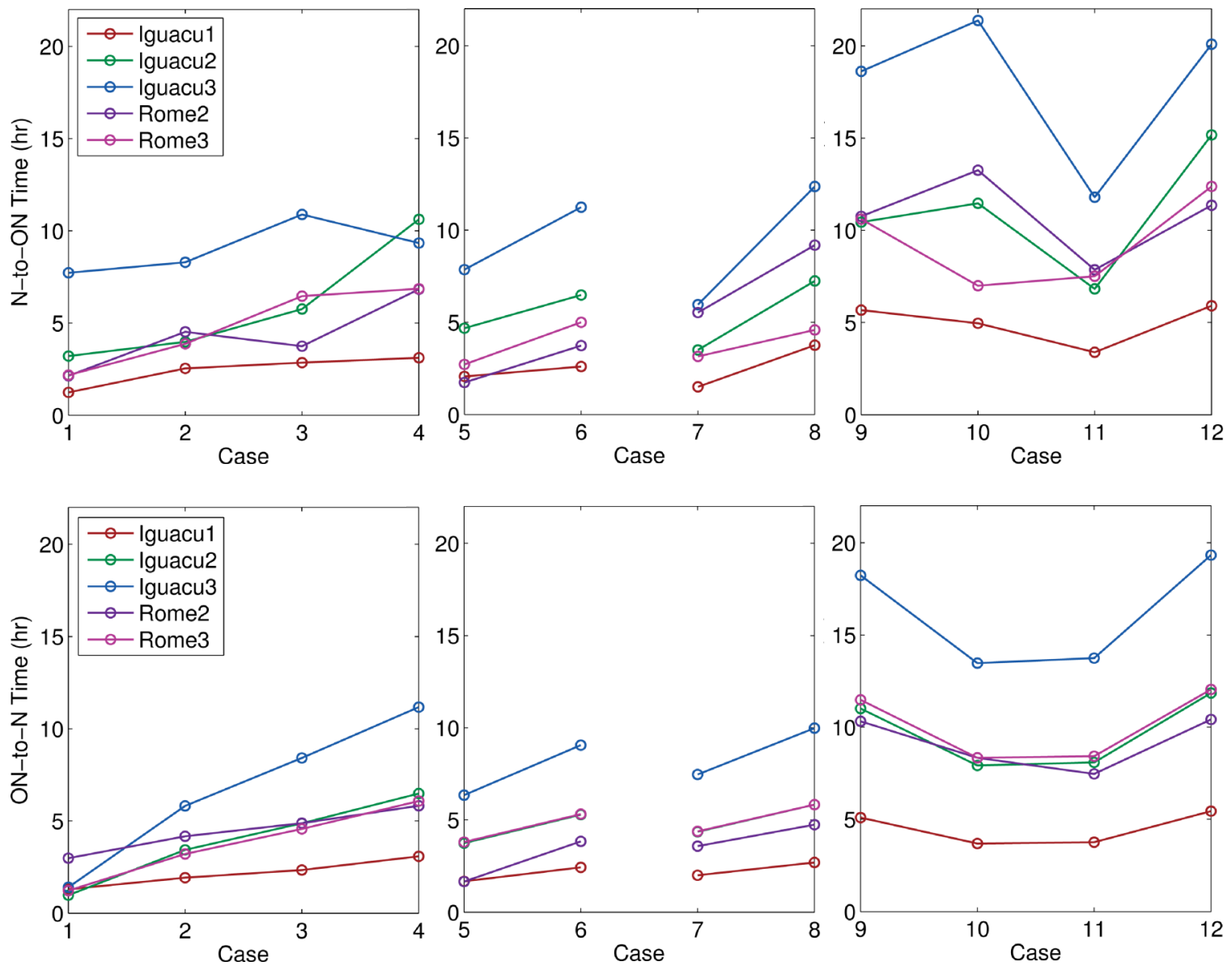

Figure 8.13: Time-frames of transitions between normal and off-normal operational modes. For most cases, the transition time is greater than the notification time for Short Notice Random Inspections. Detectable phenomena will remain in the cascade long enough for discovery using other measurement techniques. 


\section{Chapter 9}

\section{Summary and Future Work}

\subsection{Summary of Dissertation and Contributions}

The increasing demand for nuclear power has led to a taxing workload for the IAEA compounded by a stagnant budget. To continue meeting the goals of verifying the normal, peaceful behavior of gas centrifuge enrichment plants, there has been an effort to conduct intelligent, more efficient safeguards. Most of the focus in the field of safeguards has been on the development of new technologies to reduce on-site inspection activities through unattended data measurement. The work presented in this dissertation represents an ongoing effort to develop computational models of gas centrifuge enrichment cascades to understand the dynamics and capabilities of a particular plant to aid in the IAEA inspection process and to better interpret unattended monitoring data. The results of this research contribute to the general knowledge of gas centrifuge theory, cascade modeling, safeguards analyses, and nonproliferation analyses. In total, four complementary models are developed each based on issues facing the IAEA. In each case, a verification or illustrative demonstration is provided.

The first area of focus is the possibility of discrepancies between measured environmental sampling data and declared enrichment levels. These differences are most likely due to the transient startup of the cascade and to examine this behavior, an approach to predict the range of concentrations available in a physically unmodified cascade is developed. In an illustrative example, the difference between a normal range of 
enrichments due to cascade startup and an intentional misuse case is shown. This model can be used to explain anomalies in sampling results, helping to reduce the number of false alarms and on-site inspection activities.

The next focus area is due to the classified nature of centrifuge research and development. In the common case that particular details about the geometry and operating conditions of a gas centrifuge are not known, it is difficult to predict the separative performance without introducing error by estimating these parameters. To overcome this limitation, a semi-empirical centrifuge model to predict the separative performance of a gas centrifuge based on one known operating point is developed. This method is implemented in a code called CentPerform and is used to characterize the centrifuge separative power and separation factor over a broad range of centrifuge feed rates and centrifuge cuts. The model is verified by comparing with separative performance predictions from the Pancake code.

The next issue is the necessity to accurately quantify the breakout capabilities of an enrichment plant. A method is developed to study cascade interconnection and a case study on the Fuel Enrichment Plant in Iran is presented. The results of the study show the importance of including mixing inefficiencies as ideal separative work calculations can overpredict the capabilities of a plant by up to $32 \%$.

The final topic is related to the development of new safeguards approaches to reduce on-site inspection activities at enrichment plants. Unattended enrichment and feed and withdrawal cylinder weight measurement is being proposed as a viable solution to the growing demands on the IAEA safeguards system. Complementary to these technologies modeling can offer insight into the dynamics of a cascade. A transient fluid dynamics and isotope separation model is developed to simulate the normal and off-normal operation of a centrifuge cascade and the transition between the two states. The models are implemented in a code called TransCasc and are verified in a comparison with a model in the literature. Illustrative examples are used to show that there are signatures in the cascade external 
streams that indicate if off-normal operation is a result of intentional misuse of the cascade or equipment failure. Additionally, results show that for many cases in which the offnormal mode is fairly close to the normal mode, the transition time is on the order of the measurement time of an enrichment monitor. In cases where an unattended monitoring system is not installed or has failed, it is encouraging to note that the transition time for most cases is such that detectable phenomena will remain in the cascade during the announcement window of a short notice random inspection.

In addition to the findings above, the contributions of this research include:

- Three refereed journal articles (one in publication, one submitted, and one planned)

- Five conference papers

- Four codes (MultiProd, FixedPlant, CentPerform, TransCasc)

It is the hope of the author that the work detailed in this dissertation will have a positive impact on the development of new safeguards techniques and aid in the analysis of current and next generation enrichment plants.

\subsection{Future Work and Direction}

During the development of this work, several interesting topics have arisen that warrant investigation:

- Centrifuge Fluid Dynamics Modeling: In the transient cascade model developed in Chapter 8 , the hydrodynamics of the gas centrifuge were ignored because for that particular application it was deemed and verified to be unimportant. This might not always be the case, especially in extreme off-normal operational cases. At $300 \mathrm{~K}$, $\mathrm{UF}_{6}$ solidifies at roughly 130 torr. If the feed rate of the gas centrifuge is increased dramatically, the wall pressure may increase to levels where the gas might solidify on the wall in certain locations. If this happens, the imbalance may be enough to drive 
instability in the gas centrifuge and ultimately destroy the machine. In the event that the gas does solidify uniformly, the extra mass on the rotor wall could cause the centrifuge to burst.

- Enrichment Monitoring Measurement Time and Off-Normal Operation: It was mentioned that the transition time from normal to off-normal operation was on the order of the measurement time of an enrichment monitor for most of the moderate cases. The effect of transition on the enrichment measurement should be investigated. This may have implications on the effectiveness unattended monitoring systems.

- Commercial CFD Codes and Centrifuge Fluid Dynamics: One area of particular interest is modeling a gas centrifuge using a commercially available CFD code. It should be technically feasible, but there is limited information and reported success available. It would be an interesting study to conduct. 


\section{Bibliography}

[1] D. D. Eisenhower. Atoms for Peace. 470th Plenary Meeting of the United Nations General Assembly. New York, NY, December 8, 1953.

[2] D. Fischer. History of the International Atomic Energy Agency: The First Forty Years. IAEA, Vienna, Austria, 1997.

[3] IAEA Safeguards Glossary: 2001 Edition. International Atomic Energy Agency, Vienna, Austria, 2002.

[4] Treaty on the Non-Proliferation of Nuclear Weapons. http://disarmament.un.org/treaties/t/npt, Feb 2013.

[5] Nuclear Material Accounting Handbook. International Atomic Energy Agency, Vienna, Austria, May 2008.

[6] J. Cooley. The State Level Approach to International Safeguards. In Proceedings of the INMM 50th Annual Meeting, Tuscon, AZ, July 12-16, 2009.

[7] Non-Proliferation of Nuclear Weapons \& Nuclear Security. IAEA Booklet, September 2011.

[8] Nuclear Material Safeguards for Uranium Enrichment Plants. POTAS: ISPO347/R11, June 2010.

[9] J. M. Whitaker. Uranium Enrichment Plant Characteristics - a Training Manual for the IAEA. ORNL/TM-2005/43, ISOP-310/R1, May 2005.

[10] J. Cooley, N. Whiting, W. Bush, I. Tsvetkov, D. Hurt, M. Burmester, and D. Langlands. Model Safeguards Approach and Innovative Techniques Implemented by the IAEA at Gas Centrifuge Enrichment Plants. In Proceedings of the INMM 48th Annual Meeting, Tuscon, AZ, July 8-12, 2007.

[11] W. Fischer and G. Stein. On-Site Inspections: Experiences from Nuclear Safeguarding. Disarmament Forum, 1999.

[12] L. Kollar and C. E. Mathews. Evolution of Safeguards Over Time: Past, Present, and Projected Facilities, Material, and Budget. Pacific Northwest National Laboratory: PNNL-18722, July 2009.

[13] M. D. Laughter. Profile of World Uranium Enrichment Programs - 2009. ORNL/TM-2009/110, April 2009.

[14] D. Sharikov. Verification Challenges for Safeguarding Uranium Enrichment Plants. ESARDA Bulletin, 37:75-79, December 2007. 
[15] B. D. Boyer. Gas Centrifuge Enrichment Plant Safeguards: Approaches and Technology. In Proceedings of the INMM 51st Annual Meeting, Baltimore, MD, USA, 2010.

[16] B. D. Boyer. Safeguards Approaches for Gas Centrifuge Enrichment Plants. LANL Safeguards Systems Course - Pilot 2008, July 1, 2008.

[17] W. Bush, G. af Ekenstam, J. Janov, E. Kuhn, and M. Ryjinski. IAEA Experience with Environmental Sampling at Gas Centrifuge Enrichment Plants in the European Union. IAEA-SM-367/10/04, 2004.

[18] J. March-Leuba, T. Uckan, and J. Gunning. UF6 Mass Flow Measurement in Gas Centrifuge Enrichment Plants using Passive Process Monitoring. In Proceedings of the 6th American Nuclear Society International Topical Meeting on Nuclear Plant Instrumentation, Control, and Human-Machine Interface Technologies, Knoxville, TN, USA, April 5-9 2009.

[19] J. March-Leuba and T. Uckan. Fissile Flow and Enrichment Monitor for GCEP Advanced Safeguards Application. In Proceedings of the IAEA Symposium on International Safeguards: Preparing for Future Verification Challenges, Vienna, Austria, 2010.

[20] K. D. Ianakiev, B. S. Alexandrov, B. D. Boyer, T. R. Hill, D. W. MacAuthur, T. A. Marks, C. E. Moss, B. P. Nolen, M. T. Paffett, G. A. Sheppard, and M. T. Swinhoe. New Generation Enrichment Monitoring Technology for Gas Centrifuge Enrichment Plants. In Proceedings of the Nuclear Science Symposium, pages 3055 -3059, October 2008.

[21] K. D. Ianakiev, B. D. Boyer, J. M. Goda, T. R. Hill, C. E. Moss, H. Nguyen, R. F. Parker, M. T. Paffett, B. P. Nolen, and M. T. Swinhoe. Advanced Enrichment Monitoring Technology Based on Transmission Measurements With an X-Ray Source and NaI(Tl) Spectrometer. In Proceedings of the First Conference on Advancements in Nuclear Instrumentation Measurement Methods and their Applications (ANIMMA), June 2009.

[22] K. D. Ianakiev, J. M. Goda, T. R. Hill, C. E. Moss, J. J. Ong, M. T. Paffett, R. F. Parker, and M. T. Swinhoe. Advanced Technology for Enrichment Monitoring for Gas Centrifuge Enrichment Plants. Journal of Radioanalytical and Nuclear Chemistry, 282:657-661, 2009.

[23] C. A. Pickett, D. N. Kovacic, J. M. Whitaker, J. R. Younkin, J. B. Hines, M. D. Laughter, J. Morgan, B. Carrick, B. Boyer, and K. Whittle. Results from a "Proofof-Concept" Demonstration of RF-Based Tracking of UF6 Cylinders During a Processing Operation at a Uranium Enrichment Plant. In Proceedings of the INMM 49th Annual Meeting, Nashville, TN, USA, July 2008.

[24] J. Howell, P. Friend, D. Jones, and C. Taylor. Load-Cell-Based Mass Evaluation Systems Re-Assessed on the Basis of URENCO (Capenhurst) Load Cell Data. In 
Proceedings of the 31st ESARDA Annual Meeting, Vilnius, Lithuania, May 26-28, 2009.

[25] R. Carchon, L. Dechamp, L. G. Eklund, W. Janssens, G. Mercurio, P. Peerani, and P. Richir. Load Cell Monitoring in Gas Centrifuge Enrichment Plants: Potentialities for Improved Safeguard Verifications. Journal of Nuclear Engineering and Design, 241:349-356, 2011.

[26] N. C. Anheier and B. A. Bushaw. Unattended Environmental Sampling and Laser-based Enrichment Assay for Detection of Undeclared HEU Production in Enrichment Plants. In Proceedings of the Pacific Northwest International Conference on Global Nuclear Security - the Decade Ahead, April 11-16, 2010.

[27] K. A. Miller, M. T. Swinhoe, J. B. Marlow, H. O. Menlove, C. D. Rael, T. Iwamoto, T. Tamura, and S. Aiuchi. The Uranium Cylinder Assay System for Enrichment Plant Safeguards. In Proceedings of the INMM 51st Annual Meeting, Baltimore, MD, USA, July 2010.

[28] P. Friend. URENCO Conference on GCEP Safeguards. In Proceedings of the INMM 51st Annual Meeting, Baltimore, MD, USA, July 2010.

[29] L. E. Smith and A. R. Lebrun. Design, Modeling and Viability Analysis of an Online Uranium Enrichment Monitor. In Proceedings of the Nuclear Science Symposium and Medical Imaging Conference, 2011.

[30] K. R. Hase, B. D. Boyer, and H. H. Erpenbeck. Analysis of Probability of Detection of Plausible Diversion Scenarios at Gas Centrifuge Enrichment Plants using Advanced Safeguards. In Proceedings of the INMM 53rd Annual Meeting, 2012.

[31] H. G. Wood, A. Glaser, and R. S. Kemp. The Gas Centrifuge and Nuclear Weapons Proliferation. Physics Today, 61(9):40-45, 2008.

[32] A. Glaser. Characteristics of the Gas Centrifuge for Uranium Enrichment and Their Relevance for Nuclear Weapon Proliferation. Science and Global Security, 16:1-25, 2008.

[33] T. Kwak. Nuclear non-proliferation regime effectiveness: An integrated methodology for analyzing highly enriched uranium production scenarios at gas centrifuge enrichment plants. PhD thesis, Massacheusetts Institute of Technology, Cambridge, MA, USA, February 2010.

[34] K. Cohen. The Theory of Isotope Separation as Applied to the Large-Scale Production of $U$-235. National Nuclear Energy Series Division III, Vol. 1B, McGraw-Hill Book Co., New York, 1951.

[35] R. G. Hewlett and O. E. Anderson Jr. The New World, 1939/1946. Pennsylvania State University Press, 1962. 
[36] S. Whitley. Review of the Gas Centrifuge Until 1962. Part I: Principles of Separation Physics. Reviews of Modern Physics, 56(1):41-66, 1984.

[37] Soubbaramayer. Centrifugation. In S. Villani, editor, Uranium Enrichment, Topics in Applied Physics, Volume 35, Chapter 4. Springer-Verlag, New York, NY, 1979.

[38] R. L. Hoglund, J. Schacter, and E. Von Halle. Diffusion Separation Methods. Encyclopedia of Chemical Technology, 1978.

[39] D. R. Olander. Technical Basis of the Gas Centrifuge. Advances in Nuclear Science and Technology, 6:105-174, 1972.

[40] M. Benedict, T. H. Pigford, and H. Wolfgang Levi. Nuclear Chemical Engineering. McGraw Hill, 2nd edition, 1981.

[41] K. Stewartson. On Almost Rigid Rotations. Journal of Fluid Mechanics, 3:17-26, 1957.

[42] V. Barcilon and J. Pedlosky. On the Steady Motions Produced by a Stable Stratification in a Rapidly Rotating Ffluid. Journal of Fluid Mechanics, 29(4):673690, 1967.

[43] G. M. Homsy and J. L. Hudson. Centrifugally Driven Thermal Convection in a Rotating Cylinder. Journal of Fluid Mechanics, 35:33-52, 1969.

[44] G. M. Homsy and J. L. Hudson. Centrifugal Convection and its Effect on the Asymptotic Stability of a Bounded Rotating Fluid Heated From Below. Journal of Fluid Mechanics, 48:605-624, 1971.

[45] H. P. Greenspan. The Theory of Rotating Fluids. Cambridge University Press, New York, NY, 1st edition, 1968.

[46] H. Mikami. Thermally Induced Flow in Gas Centrifuge, (I). Journal of Nuclear Science and Technology, 10(7):396-401, 1973.

[47] H. Mikami. Thermally Induded Flow in a Gas Centrifuge, (II). Journal of Nuclear Science and Technology, 10(9):580-583, 1973.

[48] W. Nakayama and S. Usui. Flow in a Rotating Cylinder of a Gas Centrifuge. Journal of Nuclear Science and Technology, 11(6):242-262, 1974.

[49] T. Sakurai and T. Matsuda. Gasdynamics of a Centrifugal Machine. Journal of Fluid Mechanics, 62:727-736, 1974.

[50] T. Matsuda. Isotope Separation by Thermally Driven Countercurrent Gas Centrifuge. Journal of Nuclear Science and Technology, 12(8):512-518, 1975.

[51] T. Matsuda, K. Hashimoto, and H. Takeda. Thermally Driven Flow in a Gas Centrifuge with an Insulated Side Wall. Journal of Fluid Mechanics, 73(2):389399, 1976. 
[52] T. Matsuda and K. Hashimoto. Thermally, Mechanically or Externally Driven Flows in a Gas Centrifuge with Insulated Horizontal End Plates. Journal of Fluid Mechanics, 78(2):337-354, 1976.

[53] T. Matsuda and K. Hashimoto. The Structure of the Stewartson Layers in a Gas Centrifuge. Part 1. Insulated End Plates. Journal of Fluid Mechanics, 85(3):433442, 1978.

[54] T. Matsuda and H. Takeda. The Structure of the Stewartson Layers in a Gas Centrifuge. Part 2. Insulated Side Wall. Journal of Fluid Mechanics, 85(3):443457, 1978.

[55] T. Matsuda and K. Nakagawa. A New Type of Boundary Layer in a Rapidly Rotating Gas. Journal of Fluid Mechanics, 126:431-442, 1983.

[56] F. H. Bark and T. H. Bark. On Vertical Boundary Layers in a Rapidly Rotating Gas. Journal of Fluid Mechanics, 78(4):749-761, 1976.

[57] R. F. Gans. Poiseuille-like Flow in a Rotating Gas. Physics of Fluids, 19:1821-823, 1976.

[58] M. D. Landahl. Boundary Layers and Shear Layers in a Rapidly Rotating Gas. In Soubbaramayer, editor, Proceedings of the 2nd Workshop on Gases in Strong Rotations, Cadarache, France, April 6-8, 1977.

[59] F. H. Bark and L. S. Hultgren. On the Effects of Thermally Insulating Boundaries on Geostrophic Flows in Rapidly Rotating Gases. Journal of Fluid Mechanics, 95(1):97-118, 1979.

[60] P. Louvet and J. Durivault. Compressible Countercurrent Flow in a Strongly Rotating Cylinder. In Singular Perturbations and Boundary Layer Theory, Lecture Notes in Mathematics, Vol. 594, pages 312-333, 1977.

[61] J. J. H. Brouwers. On Compressible Flow in a Gas Centrifuge and its Effect on the Maximum Separative Power. Journal of Nuclear Technology, 39:311-322, 1978.

[62] J. J. H. Brouwers. On Compressible Flow in a Rotating Cylinder. Journal of Engineering Mathematics, 12(3):265-285, 1978.

[63] H. G. Wood. History of the SPLG Workshops. In Proceedings of the 12th Workshop on the Separation Phenomena in Liquids and Gases, 2012.

[64] H. G. Wood and J. B. Morton. Onsager's Pancake Approximation for the Fluid Dynamics of a Gas Centrifuge. Journal of Fluid Mechanics, 101(1):1-31, 1980.

[65] P. Omnes. Numerical and Physical Comparisons of Two Models of a Gas Centrifuge. Journal of Computers \& Fluids, 36(6):1028-1039, 2007.

[66] K. Van Ommen. Numerical Modeling of a Heavy Gas in Fast Rotation. PhD thesis, Twente University of Technology, Enschede, The Netherlands, 2010. 
[67] J. S. Park and J. M. Hyun. Transient Adjustment of a Gas Contained in a RapidlyRotating Infinite Cylinder. Journal of the Physical Society of Japan, 58:3949-3959, 1989.

[68] J. Park and J. Hyun. Dynamical Structure of Compressible-Fluid Flows in an Abruptly Rotating Cylinder. Journal of the Physical Society of Japan, 63:528-535, 1994.

[69] O. M. Belotserkovskii, V. B. Betelin, Borisevich V. D., V. V. Denisenko, I. V. Eriklintsev, S. A. Kozlov, A. V. Konyukhov, A. M. Oparin, and O. V. Troshkin. On the Theory of Countercurrent Flow in a Rotating Viscous Heat-Conducting Gas. Journal of Computational Mathematics and Mathematical Physics, 51(2):208-221, 2011.

[70] P. Roblin and F. Doneddu. Direct Monte-Carlo Simulations in a Gas Centrifuge. AIP Conference Proceedings, 585(1):169-173, 2001.

[71] E. Von Halle. The Countercurrent Gas Centrifuge for the Enrichment of U-235. Technical Report KOA-4058, Oak Ridge Gaseous Diffusion Plant, 1977.

[72] R.B. Bird, W.E. Stewart, and E.N. Lightfoot. Transport Phenomena. John Wiley \& Sons, Inc., 2nd edition, 2007.

[73] W. Groth. Gas Centrifuges. In H. London, editor, Separation of Isotopes. George Newnes Limited, 1961.

[74] G. F. Carrier and S. H. Maslen. Flow Phenomena in Rapidly Rotating Systems. Technical report, USAEC Report TID-18065, 1962.

[75] G. F. Carrier. Phenomena in Rotating Fluids. In Proceedings of the 11th International Congress on Applied Mechanics, Munich, Germany, 1964.

[76] H. G. Wood and G. Sanders. Rotating Compressible Flows with Internal Sources and Sinks. Journal of Fluid Mechanics, 127:299-313, 1983.

[77] M. D. Gunzburger and H. G. Wood. A Finite Element Method for the Onsager Pancake Equation. Journal of Computer Methods in Applied Mechanics and Engineering, 31(1):43-59, 1982.

[78] R. J. Ribando. A Finite-Difference Solution of Onsager's Model for Flow in a Gas Centrifuge. Journal of Computers \& Fluids, 12(3):235-252, 1984.

[79] M. De Stadler and K. Chand. A Finite-Difference Numerical Method for Onsager's Pancake Approximation for Fluid Flow in a Gas Centrifuge. Lawrence Livermore National Laboratory, UCRL-TR-236581, 2007.

[80] J. Hu, C. Ying, and S. Zeng. Overall Separation Factor in a Gas Centrifuge Using a Purely Axial Flow Model. Separation Science and Technology, 40(10):2139-2152, 2005. 
[81] E. Von Halle. Centrifuge Cascade Program (CenCas). Technical report, ORNL X/GID-08-001, 2008.

[82] W. H. Furry, R. Clark Jones, and L. Onsager. On the Theory of Isotope Separation by Thermal Diffusion. Physical Review, 55(11):1083-1095, 1939.

[83] Third Workshop on Gases in Strong Rotation, Rome, Italy, March 27-29, 1979.

[84] C. Schwab, N. A. S. Rodrigues, and H. G. Wood, editors. Proceedings of the 5th Workshop on Separation Phenomena in Liquids and Gases, Foz do Iguaçu, Brazil, 1996.

[85] B. Brigoli. Cascade Theory. In Stelio Villani, editor, Uranium Enrichment, Topics in Applied Physics, Volume 35, Chapter 2, pages 13-54. Springer-Verlag, New York, NY, 1979.

[86] E. Von Halle. Stage Separation Theory. Von Karman Institute of Fluid Dynamics 1977-1978 Lecture Series on Aerodynamic Separation of Gases and Isotopes, May 29, 1977.

[87] H. A. Elayat, W.J. O'Connel, and B. D. Boyer. Gas Centrifuge Enrichment Plant Safeguards System Modeling. In Proceedings of the INMM 47th Annual Meeting, Nashville, TN, USA, July 2006.

[88] J. F. A. Delbeke. Theoretical Analysis to Assess the Separative Power of Reconfigured Cascades of Predesigned Gas Centrifuges. Journal of Industrial and Engineering Chemistry Research, 48(10):4960-4965, 2009.

[89] J. F. A. Delbeke, G. Eklund, B. P. M. van Esch, G. Janssens-Maenhout, and W. Janssens. Verifying the Spin of Centrifuges. Journal of Energy, 35(8):31233130, August 2010.

[90] J. F. A. Delbeke, J. Howell, and W. Janssens. The Detection of Undeclared LEU Production at a GCEP by Real-Time Mass Balancing. In Proceedings of the INMM 48th Annual Meeting, Tucson, AZ, USA, 2007.

[91] J. F. A. Delbeke, J. Howell, G. Eklund, P. Peerani, and W. Janssens. The Real Time Mass Evaluation System as a Tool for Detection of Undeclared Cascade Operation at GCEPs. In Proceedings of the 8th International Conference on Facility OperationsSafeguards Interface, Portland, OR, USA, 2008.

[92] C. Cleary and R. Carchon. Safeguards Analysis of Material Flows in a Gas Centrifuge Enrichment Plant. ESARDA Bulletin, 41:31-37, June 2009.

[93] P. G. Rousseau, B. du Toit, W. A. Landman, A. J. K van de Walt, D. J. Viljoen, and G. J. Leuner. Dynamic Simulation of a Centrifuge Cascade Plant with the Aid of a Flow Network Simulation Code. In Proceedings of the 12th Workshop on Separation Phenomena in Liquids and Gases, June 3-8, 2012. 
[94] P. J. Migliorini and H. G. Wood. A Study of Multicomponent Streams in Off-Design Centrifuge Cascades. Separation Science and Technology, 47(7):921-928, 2012.

[95] A. de la Garza, G.A. Garrett, and J.E. Murphy. Multicomponent Isotope Separation in Cascades. Chemical Engineering Science, 15(3-4):188-209, 1961.

[96] A. de la Garza. A Generalization of the Matched Abundance-Ratio Cascade for Multicomponent Isotope Separation. Chemical Engineering Science, 18(2):73-82, 1963.

[97] E. Von Halle. Multicomponent Isotope Separation in Matched Abundance Ratio Cascades Composed of Stages with Large Separation Factors. In K. G. Roesner, editor, Proceedings of the 1st Workshop on Separation Phenomena in Liquids and Gases, pages 325-356, Darmstadt, Germany, July 20-23 1987.

[98] C. Ying, Z. Guo, and H. G. Wood. Solution of the Diffusion Equations in a Gas Centrifuge for Separation of Multicomponent Mixtures. Separation Science and Technology, 31(18):2455-2471, 1996.

[99] D. Albright, P. Brannan, A. Stricker, and C. Walrond. ISIS Analysis of the IAEA Iran Safeguards Report from September 2, 2011. ISIS Report, September 2, 2011.

[100] D. Albright and C. Walrond. Performance of the IR-1 Centrifuge at Natanz. ISIS Report, October 18, 2011.

[101] Implementation of the NPT Safeguards Agreement and Relevant Provisions of Security Council Resolutions in the Islamic Republic of Iran. GOV/2011/29, May 24, 2011.

[102] W. C. Witt, C. Walrond, D. Albright, and H. G. Wood. Iran's Evolving Breakout Potential. ISIS Report, October 8, 2012.

[103] I. Barzashka. Using Enrichment Capacity to Estimate Iran's Breakout Potential. Federation of the American Scientists Issue Brief, January 21, 2011.

[104] H. G. Wood and P. J. Migliorini. Fixed Plant Proliferation Analysis of Iran's Natanz Plant. In Proceedings of the INMM 52nd Annual Meeting, Orlando, FL, USA, 2012.

[105] ISIS Nuclear Iran: Nuclear History, Accessed: February 12, 2013. http://www.isisnucleariran.org/nuclear-history/.

[106] D. Albright. Peddling Peril: How the Secret Nuclear Trade Arms America's Enemies. Free Press, 2010.

[107] Implementation of the NPT Safeguards Agreement and Relevant Provisions of Security Council Resolutions in the Islamic Republic of Iran. GOV/2012/9, February 24, 2012. 
[108] P. J. Migliorini and H. G. Wood. Transient Fluid Dynamics Modeling of Gas Centrifuge Enrichment Plants. In Proceedings of the 52nd Annual Meeting of the Institute of Nuclear Materials Management, Palm Desert, CA, USA, July 17-21, 2011.

[109] P. J. Migliorini and H. G. Wood. Transient Operation of a Gas Centrifuge Cascade to Determine Proliferation Time Frames. In Proceedings of the 12th Workshop on Separation Phenomena in Liquids and Gases, Paris, France, June 3-8, 2012.

[110] P. J. Migliorini and H. G. Wood. Transient Isotope Separation Modeling of Gas Centrifuge Enrichment Plants. In Proceedings of the 53rd Annual Meeting of the Institute of Nuclear Materials Management, Orlando, FL, USA, July 15-19, 2012.

[111] R. DeWitt. Uranium Hexafluoride: A Survey of the Physicochemical Properties. Technical Report GAT-280, Goodyear Atomic Corporation, Portsmouth, Ohio, 1960.

[112] MATLAB Product Help. Verison 2010b, 2010.

[113] L. Zarkova and U. Hohm. pVT-Second Virial Coefficients $B$, Viscosity $\eta$, and Self-Diffusion $\rho D$ of the Gases: BF3, CF4, SiF4, CCl4, SiCl4, SF6, MoF6, WF6, UF6, C(CH3)4, and Si(CH3)4 Determined by Means of an Isotropic TemperatureDependent Potential. Journal of Physical and Chemical Reference Data, 31(1):183216, 2002.

[114] S. Zeng and C. Ying. A Robust and Efficient Calculation Procedure for Determining Concentration Distribution of Multicomponent Mixtures. Separation Science and Technology, 35(4):613-622, 2000.

[115] S. Zeng and C. Ying. A Second-Order Time-Accurate Method for Determination of Concentration Distribution of Multicomponent Mixtures in Separation Cascades. Separation Science and Technology, 35(5):729-741, 2000.

[116] S. Zeng and C. Ying. Transient Process in Gas Centrifuge Cascades for Separation of Multicomponent Isotope Mixtures. Separation Science and Technology, 36(15):3439-3457, 2001.

[117] P. Friend. Continuous Enrichment Monitoring Trial. In GCEP Technology Stakeholders Meeting, Charlottesville, VA, USA, May 17, 2012. 


\section{Appendix A:}

\section{Coefficients of Pure Axial Flow Model}

Let

$$
\begin{aligned}
& a_{1}=\operatorname{Ei}\left(A^{2}\right), \quad a_{2}=0, \quad a_{3}=1 \\
& b_{1}=-\frac{e^{A^{2}(1-\varepsilon)}}{A^{2}(1-\varepsilon)}, \quad b_{2}=-\frac{1}{A^{2}(1-\varepsilon)}, \quad b_{3}=0 \\
& g_{1}=e^{-A^{2}} \operatorname{Ei}\left[2 A^{2}(1-\varepsilon)\right]-e^{-\varepsilon A^{2}} \operatorname{Ei}\left[A^{2}(1-\varepsilon)\right]-e^{-A^{2}} \operatorname{Ei}\left[2 A^{2}\right]+\operatorname{Ei}\left[A^{2}\right] \\
& g_{2}=e^{-A^{2}} \operatorname{Ei}\left[A^{2}(1-\varepsilon)\right]-e^{-\varepsilon A^{2}} \ln [1-\varepsilon]-e^{-A^{2}} \operatorname{Ei}\left[A^{2}\right] \\
& g_{3}=-e^{-\varepsilon A^{2}}+1 \\
& f_{1}=\zeta A^{2} \\
& f_{2}=\zeta A^{2} e^{-\varepsilon A^{2}} \\
& f_{3}=Q+\frac{1}{2} \zeta A^{2}\left[-e^{-2 \varepsilon A^{2}}+1\right]
\end{aligned}
$$

then

$$
\begin{gathered}
c_{1}=\frac{a_{2} b_{3} f_{3}-a_{3} b_{2} f_{3}-a_{2} f_{2} g_{3}+a_{3} f_{2} g_{2}+b_{2} f_{1} g_{3}-b_{3} f_{1} g_{2}}{a_{1} b_{2} g_{3}-a_{1} b_{3} g_{2}-a_{2} b_{1} g_{3}+a_{2} b_{3} g_{1}+a_{3} b_{1} g_{2}-a_{3} b_{2} g_{1}} \\
c_{2}=-\frac{a_{1} b_{3} f_{3}-a_{3} b_{1} f_{3}-a_{1} f_{2} g_{3}+a_{3} f_{2} g_{1}+b_{1} f_{1} g_{3}-b_{3} f_{1} g_{1}}{a_{1} b_{2} g_{3}-a_{1} b_{3} g_{2}-a_{2} b_{1} g_{3}+a_{2} b_{3} g_{1}+a_{3} b_{1} g_{2}-a_{3} b_{2} g_{1}} \\
c_{3}=\frac{a_{1} b_{2} f_{3}-a_{2} b_{1} f_{3}-a_{1} f_{2} g_{2}+a_{2} f_{2} g_{1}+b_{1} f_{1} g_{2}-b_{2} f_{1} g_{1}}{a_{1} b_{2} g_{3}-a_{1} b_{3} g_{2}-a_{2} b_{1} g_{3}+a_{2} b_{3} g_{1}+a_{3} b_{1} g_{2}-a_{3} b_{2} g_{1}}
\end{gathered}
$$




\section{Appendix B:}

\section{Full Cascade Details for Transient Study}

Table B.1: Stage details for Iguaçu1

\begin{tabular}{ccccccccccc}
\hline$n$ & $M$ & $D$ & $G$ & $L$ & $\theta$ & $N^{\prime \prime}$ & $N$ & $N^{\prime}$ & $\delta U$ & $\gamma_{0}$ \\
- & - & $\mathrm{kgU} / \mathrm{yr}$ & $\mathrm{kgU} / \mathrm{yr}$ & $\mathrm{kgU} / \mathrm{yr}$ & - & at. frac. & at. frac. & at. frac. & SWU/yr & - \\
\hline 1 & 6 & 1874 & 3536 & 1662 & 0.470 & 0.0035 & 0.0039 & 0.0044 & 26.24 & 1.276 \\
2 & 11 & 3536 & 6671 & 3135 & 0.470 & 0.0039 & 0.0044 & 0.0050 & 48.12 & 1.272 \\
3 & 16 & 5009 & 9452 & 4443 & 0.470 & 0.0044 & 0.0050 & 0.0056 & 69.98 & 1.276 \\
4 & 20 & 6317 & 11921 & 5604 & 0.470 & 0.0050 & 0.0056 & 0.0063 & 87.48 & 1.275 \\
5 & 23 & 7478 & 14112 & 6634 & 0.470 & 0.0056 & 0.0063 & 0.0071 & 100.55 & 1.270 \\
6 & 27 & 8508 & 16059 & 7550 & 0.470 & 0.0063 & 0.0071 & 0.0080 & 118.10 & 1.275 \\
7 & 23 & 7325 & 13827 & 6502 & 0.470 & 0.0071 & 0.0080 & 0.0090 & 100.62 & 1.273 \\
8 & 20 & 6277 & 11850 & 5573 & 0.470 & 0.0080 & 0.0090 & 0.0102 & 87.48 & 1.276 \\
9 & 17 & 5348 & 10097 & 4749 & 0.470 & 0.0090 & 0.0102 & 0.0115 & 74.36 & 1.275 \\
10 & 14 & 4524 & 8543 & 4019 & 0.470 & 0.0102 & 0.0115 & 0.0129 & 61.24 & 1.271 \\
11 & 12 & 3794 & 7166 & 3372 & 0.471 & 0.0115 & 0.0129 & 0.0146 & 52.50 & 1.274 \\
12 & 10 & 3147 & 5944 & 2797 & 0.471 & 0.0129 & 0.0146 & 0.0164 & 43.75 & 1.275 \\
13 & 8 & 2572 & 4860 & 2288 & 0.471 & 0.0146 & 0.0164 & 0.0185 & 35.01 & 1.272 \\
14 & 6 & 2063 & 3898 & 1836 & 0.471 & 0.0164 & 0.0183 & 0.0205 & 26.11 & 1.261 \\
15 & 5 & 1611 & 3045 & 1434 & 0.471 & 0.0182 & 0.0204 & 0.0230 & 21.88 & 1.271 \\
16 & 4 & 1209 & 2286 & 1077 & 0.471 & 0.0203 & 0.0229 & 0.0258 & 17.49 & 1.281 \\
17 & 3 & 852 & 1612 & 760 & 0.471 & 0.0227 & 0.0257 & 0.0291 & 13.09 & 1.291 \\
18 & 2 & 535 & 1012 & 477 & 0.472 & 0.0256 & 0.0291 & 0.0330 & 8.67 & 1.300 \\
19 & 1 & 252 & 477 & 225 & 0.472 & 0.0290 & 0.0330 & 0.0376 & 4.30 & 1.309 \\
\hline
\end{tabular}


Table B.2: Stage details for Iguaçu2

\begin{tabular}{ccccccccccc}
\hline$n$ & $M$ & $D$ & $G$ & $L$ & $\theta$ & $N^{\prime \prime}$ & $N$ & $N^{\prime}$ & $\delta U$ & $\gamma_{0}$ \\
- & - & $\mathrm{kgU} / \mathrm{yr}$ & $\mathrm{kgU} / \mathrm{yr}$ & $\mathrm{kgU} / \mathrm{yr}$ & - & at. frac. & at. frac. & at. frac. & SWU/yr & - \\
\hline 1 & 59 & 18739 & 35356 & 16617 & 0.470 & 0.0035 & 0.0039 & 0.0044 & 258.20 & 1.274 \\
2 & 111 & 35356 & 66710 & 31354 & 0.470 & 0.0039 & 0.0044 & 0.0050 & 485.79 & 1.273 \\
3 & 157 & 50094 & 94524 & 44430 & 0.470 & 0.0044 & 0.0050 & 0.0056 & 687.06 & 1.273 \\
4 & 198 & 63170 & 119207 & 56037 & 0.470 & 0.0050 & 0.0056 & 0.0063 & 866.49 & 1.273 \\
5 & 234 & 74776 & 141120 & 66344 & 0.470 & 0.0056 & 0.0063 & 0.0071 & 1023.93 & 1.273 \\
6 & 267 & 85083 & 160587 & 75504 & 0.470 & 0.0064 & 0.0072 & 0.0080 & 1168.56 & 1.273 \\
7 & 230 & 73253 & 138273 & 65020 & 0.470 & 0.0071 & 0.0080 & 0.0090 & 1006.66 & 1.273 \\
8 & 197 & 62769 & 118497 & 55728 & 0.470 & 0.0080 & 0.0090 & 0.0102 & 862.22 & 1.273 \\
9 & 168 & 53477 & 100969 & 47492 & 0.470 & 0.0090 & 0.0102 & 0.0115 & 735.31 & 1.273 \\
10 & 142 & 45241 & 85431 & 40190 & 0.470 & 0.0102 & 0.0115 & 0.0129 & 621.51 & 1.273 \\
11 & 119 & 37940 & 71655 & 33716 & 0.471 & 0.0115 & 0.0129 & 0.0146 & 520.82 & 1.273 \\
12 & 99 & 31465 & 59438 & 27973 & 0.471 & 0.0129 & 0.0146 & 0.0164 & 433.33 & 1.273 \\
13 & 81 & 25723 & 48601 & 22878 & 0.471 & 0.0146 & 0.0164 & 0.0185 & 354.55 & 1.274 \\
14 & 65 & 20628 & 38984 & 18356 & 0.471 & 0.0164 & 0.0185 & 0.0208 & 284.52 & 1.274 \\
15 & 51 & 16106 & 30446 & 14340 & 0.471 & 0.0185 & 0.0208 & 0.0234 & 223.22 & 1.274 \\
16 & 38 & 12090 & 22861 & 10771 & 0.471 & 0.0208 & 0.0234 & 0.0263 & 166.35 & 1.273 \\
17 & 27 & 8521 & 16117 & 7597 & 0.471 & 0.0234 & 0.0263 & 0.0296 & 118.19 & 1.274 \\
18 & 17 & 5346 & 10117 & 4770 & 0.472 & 0.0263 & 0.0296 & 0.0333 & 74.41 & 1.275 \\
19 & 8 & 2520 & 4770 & 2251 & 0.472 & 0.0296 & 0.0333 & 0.0375 & 35.02 & 1.275 \\
\hline
\end{tabular}

Table B.3: Stage details for Iguaçu3

\begin{tabular}{ccccccccccc}
\hline$n$ & $M$ & $D$ & $G$ & $L$ & $\theta$ & $N^{\prime \prime}$ & $N$ & $N^{\prime}$ & $\delta U$ & $\gamma_{0}$ \\
- & - & $\mathrm{kgU} / \mathrm{yr}$ & $\mathrm{kgU} / \mathrm{yr}$ & $\mathrm{kgU} / \mathrm{yr}$ & - & at. frac. & at. frac. & at. frac. & SWU/yr & - \\
\hline 1 & 587 & 187395 & 353560 & 166165 & 0.470 & 0.0035 & 0.0039 & 0.0044 & 2568.71 & 1.273 \\
2 & 1108 & 353560 & 667104 & 313544 & 0.470 & 0.0039 & 0.0044 & 0.0050 & 4848.77 & 1.273 \\
3 & 1571 & 500939 & 945243 & 444304 & 0.470 & 0.0044 & 0.0050 & 0.0056 & 6875.30 & 1.273 \\
4 & 1981 & 631699 & 1192065 & 560367 & 0.470 & 0.0050 & 0.0056 & 0.0063 & 8669.63 & 1.273 \\
5 & 2345 & 747761 & 1411200 & 663439 & 0.470 & 0.0056 & 0.0063 & 0.0071 & 10262.69 & 1.273 \\
6 & 2668 & 850833 & 1605869 & 755035 & 0.470 & 0.0064 & 0.0072 & 0.0080 & 11676.26 & 1.273 \\
7 & 2298 & 732530 & 1382726 & 650196 & 0.470 & 0.0071 & 0.0080 & 0.0090 & 10057.35 & 1.273 \\
8 & 1969 & 627690 & 1184969 & 557278 & 0.470 & 0.0080 & 0.0090 & 0.0102 & 8617.49 & 1.273 \\
9 & 1678 & 534773 & 1009690 & 474917 & 0.470 & 0.0091 & 0.0102 & 0.0115 & 7344.10 & 1.273 \\
10 & 1420 & 452412 & 854313 & 401901 & 0.470 & 0.0102 & 0.0115 & 0.0130 & 6215.10 & 1.273 \\
11 & 1191 & 379396 & 716553 & 337157 & 0.471 & 0.0115 & 0.0130 & 0.0146 & 5212.91 & 1.273 \\
12 & 988 & 314651 & 594384 & 279732 & 0.471 & 0.0130 & 0.0146 & 0.0164 & 4324.52 & 1.273 \\
13 & 808 & 257227 & 486010 & 228783 & 0.471 & 0.0146 & 0.0164 & 0.0185 & 3536.79 & 1.273 \\
14 & 648 & 206278 & 389839 & 183561 & 0.471 & 0.0164 & 0.0185 & 0.0208 & 2836.49 & 1.273 \\
15 & 506 & 161056 & 304456 & 143400 & 0.471 & 0.0185 & 0.0208 & 0.0234 & 2214.96 & 1.273 \\
16 & 380 & 120895 & 228606 & 107711 & 0.471 & 0.0208 & 0.0234 & 0.0264 & 1663.49 & 1.273 \\
17 & 268 & 85205 & 161173 & 75968 & 0.471 & 0.0235 & 0.0264 & 0.0297 & 1173.28 & 1.273 \\
18 & 168 & 53462 & 101167 & 47704 & 0.472 & 0.0264 & 0.0297 & 0.0334 & 735.46 & 1.273 \\
19 & 79 & 25199 & 47704 & 22505 & 0.472 & 0.0297 & 0.0334 & 0.0375 & 345.79 & 1.273 \\
\hline
\end{tabular}


Table B.4: Stage details for Rome1

\begin{tabular}{ccccccccccc}
\hline$n$ & $M$ & $D$ & $G$ & $L$ & $\theta$ & $N^{\prime \prime}$ & $N$ & $N^{\prime}$ & $\delta U$ & $\gamma_{0}$ \\
- & - & $\mathrm{kgU} / \mathrm{yr}$ & $\mathrm{kgU} / \mathrm{yr}$ & $\mathrm{kgU} / \mathrm{yr}$ & - & at. frac. & at. frac. & at. frac. & SWU/yr & - \\
\hline 1 & 5 & 16605 & 31144 & 14539 & 0.467 & 0.0032 & 0.0036 & 0.0042 & 268.37 & 1.301 \\
2 & 10 & 31144 & 58418 & 27274 & 0.467 & 0.0036 & 0.0042 & 0.0048 & 537.54 & 1.312 \\
3 & 14 & 43879 & 82311 & 38432 & 0.467 & 0.0042 & 0.0048 & 0.0055 & 752.50 & 1.311 \\
4 & 17 & 55037 & 103251 & 48214 & 0.467 & 0.0048 & 0.0055 & 0.0062 & 913.35 & 1.305 \\
5 & 20 & 64819 & 121613 & 56795 & 0.467 & 0.0055 & 0.0062 & 0.0071 & 1074.48 & 1.305 \\
6 & 23 & 73400 & 137728 & 64329 & 0.467 & 0.0063 & 0.0072 & 0.0081 & 1235.99 & 1.308 \\
7 & 19 & 62304 & 116922 & 54619 & 0.467 & 0.0071 & 0.0081 & 0.0093 & 1020.27 & 1.303 \\
8 & 16 & 52594 & 98714 & 46121 & 0.467 & 0.0081 & 0.0092 & 0.0105 & 859.08 & 1.302 \\
9 & 14 & 44096 & 82778 & 38682 & 0.467 & 0.0092 & 0.0105 & 0.0120 & 752.42 & 1.310 \\
10 & 11 & 36657 & 68827 & 32170 & 0.467 & 0.0105 & 0.0120 & 0.0137 & 590.28 & 1.300 \\
11 & 9 & 30145 & 56611 & 26467 & 0.468 & 0.0119 & 0.0136 & 0.0155 & 482.85 & 1.299 \\
12 & 8 & 24442 & 45912 & 21471 & 0.468 & 0.0135 & 0.0154 & 0.0177 & 430.06 & 1.316 \\
13 & 6 & 19446 & 36538 & 17092 & 0.468 & 0.0154 & 0.0176 & 0.0200 & 322.30 & 1.305 \\
14 & 5 & 15067 & 28319 & 13252 & 0.468 & 0.0175 & 0.0200 & 0.0229 & 268.81 & 1.318 \\
15 & 4 & 11227 & 21110 & 9883 & 0.468 & 0.0200 & 0.0230 & 0.0264 & 214.94 & 1.331 \\
16 & 2 & 7857 & 14780 & 6923 & 0.468 & 0.0231 & 0.0260 & 0.0292 & 106.23 & 1.271 \\
17 & 2 & 4897 & 9216 & 4319 & 0.469 & 0.0251 & 0.0291 & 0.0337 & 106.92 & 1.357 \\
18 & 1 & 2294 & 4319 & 2025 & 0.469 & 0.0289 & 0.0337 & 0.0392 & 53.26 & 1.370 \\
\hline
\end{tabular}

Table B.5: Stage details for Rome2

\begin{tabular}{ccccccccccc}
\hline$n$ & $M$ & $D$ & $G$ & $L$ & $\theta$ & $N^{\prime \prime}$ & $N$ & $N^{\prime}$ & $\delta U$ & $\gamma_{0}$ \\
- & - & $\mathrm{kgU} / \mathrm{yr}$ & $\mathrm{kgU} / \mathrm{yr}$ & $\mathrm{kgU} / \mathrm{yr}$ & - & at. frac. & $\begin{array}{c}\text { at. frac. } \\
\text { at. frac. }\end{array}$ & $\begin{array}{c}\text { SWU/yr } \\
\text { SW }\end{array}$ \\
\hline 1 & 52 & 166049 & 311442 & 145393 & 0.467 & 0.0032 & 0.0036 & 0.0042 & 2794.48 & 1.308 \\
2 & 97 & 311442 & 584180 & 272738 & 0.467 & 0.0036 & 0.0042 & 0.0048 & 5212.45 & 1.307 \\
3 & 137 & 438787 & 823106 & 384319 & 0.467 & 0.0042 & 0.0048 & 0.0054 & 7362.06 & 1.307 \\
4 & 172 & 550368 & 1032505 & 482137 & 0.467 & 0.0048 & 0.0054 & 0.0062 & 9242.92 & 1.307 \\
5 & 203 & 648186 & 1216132 & 567947 & 0.467 & 0.0054 & 0.0062 & 0.0071 & 10908.96 & 1.308 \\
6 & 230 & 733995 & 1377282 & 643286 & 0.467 & 0.0063 & 0.0071 & 0.0081 & 12359.87 & 1.308 \\
7 & 195 & 623035 & 1169222 & 546187 & 0.467 & 0.0071 & 0.0081 & 0.0093 & 10478.80 & 1.307 \\
8 & 165 & 525936 & 987142 & 461207 & 0.467 & 0.0081 & 0.0093 & 0.0106 & 8866.80 & 1.308 \\
9 & 138 & 440955 & 827777 & 386822 & 0.467 & 0.0093 & 0.0106 & 0.0121 & 7415.59 & 1.307 \\
10 & 115 & 366570 & 688268 & 321698 & 0.467 & 0.0106 & 0.0121 & 0.0138 & 6179.73 & 1.308 \\
11 & 94 & 301447 & 566114 & 264667 & 0.468 & 0.0121 & 0.0138 & 0.0157 & 5050.86 & 1.307 \\
12 & 77 & 244416 & 459123 & 214707 & 0.468 & 0.0138 & 0.0157 & 0.0180 & 4137.76 & 1.308 \\
13 & 61 & 194455 & 365376 & 170920 & 0.468 & 0.0158 & 0.0180 & 0.0205 & 3277.76 & 1.308 \\
14 & 47 & 150669 & 283192 & 132523 & 0.468 & 0.0180 & 0.0205 & 0.0234 & 2525.29 & 1.307 \\
15 & 35 & 112272 & 211098 & 98826 & 0.468 & 0.0205 & 0.0234 & 0.0266 & 1880.48 & 1.306 \\
16 & 25 & 78575 & 147800 & 69225 & 0.468 & 0.0234 & 0.0267 & 0.0304 & 1343.42 & 1.310 \\
17 & 15 & 48974 & 92163 & 43189 & 0.469 & 0.0267 & 0.0304 & 0.0345 & 805.40 & 1.303 \\
18 & 7 & 22938 & 43189 & 20251 & 0.469 & 0.0304 & 0.0345 & 0.0392 & 375.78 & 1.302 \\
\hline
\end{tabular}

